\title{
Article \\ Optic Atrophy 1 Controls Human Neuronal Development by Preventing Aberrant Nuclear DNA Methylation
}

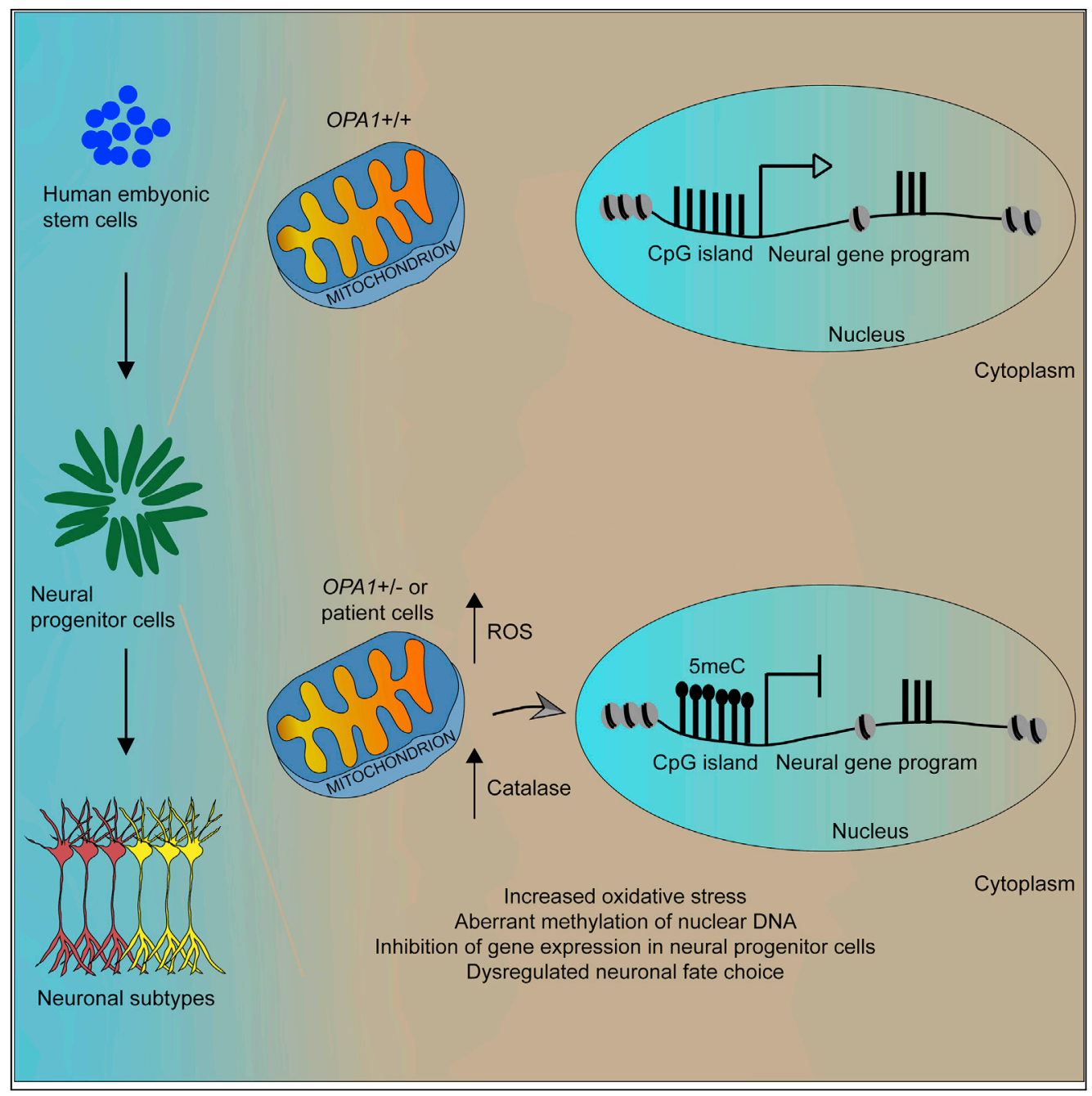

Safak Caglayan, Adnan Hashim, Artur Cieslar-

Pobuda, ....

Antonella Boschi, Kjetil Taskén, Judith Staerk

judith.staerk@ncmm.uio.no

HIGHLIGHTS

OPA1 haploinsufficiency impairs formation of DLX1/2-positive

GABAergic neurons

Reduced OPA1 levels significantly alter the transcriptional circuitry in neural cells

Expression of the pioneer factor FOXG1 is decreased in OPA $1+/$ neural progenitor cells

Impaired FOXG1

expression correlates with increased CpG methylation at its promoter 


\title{
Optic Atrophy 1 Controls Human Neuronal Development by Preventing Aberrant Nuclear DNA Methylation
}

\author{
Safak Caglayan, ${ }^{1}$ Adnan Hashim, ${ }^{1}$ Artur Cieslar-Pobuda, ${ }^{1}$ Vidar Jensen, ${ }^{2}$ Sidney Behringer, ${ }^{3}$ Burcu Talug, ${ }^{1}$ \\ Dinh Toi Chu, ${ }^{1}$ Christian Pecquet, ${ }^{4,5}$ Marie Rogne, ${ }^{1}$ Andreas Brech, ${ }^{6}$ Sverre Henning Brorson, ${ }^{7}$ \\ Erlend Arnulf Nagelhus, ${ }^{2}$ Luciana Hannibal, ${ }^{3}$ Antonella Boschi, ${ }^{8}$ Kjetil Taskén, $1,9,10$ and Judith Staerk $111,12, *$
}

\section{SUMMARY}

Optic atrophy 1 (OPA1), a GTPase at the inner mitochondrial membrane involved in regulating mitochondrial fusion, stability, and energy output, is known to be crucial for neural development: Opa1 heterozygous mice show abnormal brain development, and inactivating mutations in OPA1 are linked to human neurological disorders. Here, we used genetically modified human embryonic and patientderived induced pluripotent stem cells and reveal that OPA1 haploinsufficiency leads to aberrant nuclear DNA methylation and significantly alters the transcriptional circuitry in neural progenitor cells (NPCs). For instance, expression of the forkhead box G1 transcription factor, which is needed for GABAergic neuronal development, is repressed in OPA1+/- NPCs. Supporting this finding, OPA1+/ - NPCs cannot give rise to GABAergic interneurons, whereas formation of glutamatergic neurons is not affected. Taken together, our data reveal that OPA1 controls nuclear DNA methylation and expression of key transcription factors needed for proper neural cell specification.

\section{INTRODUCTION}

Mitochondria are double-membrane-bound subcellular organelles required for numerous cellular functions. In response to metabolic and physiological signals, mitochondria undergo fusion and fission, processes that are catalyzed by conserved GTP-hydrolyzing enzymes and their associated proteins (Chan, 2012). Fission of mitochondria is needed for their fragmentation. This is triggered by dynamin 1-like protein (DNM1L), a cytoplasmic protein that is recruited to the mitochondrial surface where it constricts mitochondrial tubules. Fusion of the outer membranes is catalyzed by mitofusin 1 and 2 (MFN1, MFN2), whereas fusion of the mitochondrial inner membranes is catalyzed by the mitochondrial dynamin-like GTPase optic atrophy 1 (OPA1). Due to RNA splicing and protein processing, different isoforms of OPA1 exist. The long isoforms localize to the mitochondrial inner membrane through their transmembrane domains, whereas the short isoforms are expressed as soluble proteins in the mitochondrial intermembrane space. A balance of long and short OPA1 isoforms is required to maintain a healthy mitochondrial network (Del Dotto et al., 2017; MacVicar and Langer, 2016).

OPA1 is crucial for tissue development. Opa1 heterozygous animals are viable, but experience a progressive loss of retinal ganglion cells (RGCs), optic nerve degeneration, and abnormal brain development (Davies et al., 2007; Alavi et al., 2007), whereas Opa1-null mice die early during embryonic development due to growth retardation and morphological abnormalities (Davies et al., 2007; Alavi et al., 2007). In humans, heterozygous mutations in OPA 1 cause the most common form of autosomal dominant optic atrophy (ADOA), a neuropathy wherein the majority of patients experience impaired vision (Delettre et al., 2000; Alexander et al., 2000). Homozygous mutations in OPA1 result in severe and fatal infantile disorders with neurodevelopmental deficits, multi-organ complications, encephalopathy, cardiomyopathy, and optic atrophy (Spiegel et al., 2016; Nasca et al., 2017).

To elucidate the molecular mechanisms by which OPA1 contributes to human neural development, we used OPA 1 haploinsufficient pluripotent stem cell lines and differentiated them into neural progenitor cells
${ }^{1}$ Centre for Molecular Medicine Norway, Nordic EMBL Partnership, University of Oslo and Oslo University Hospital, 0318 Oslo, Norway

${ }^{2}$ GliaLab and Letten Centre, Division of Physiology, Department of Molecular Medicine, Institute of Basic Medical Sciences, University of Oslo, 0317 Oslo, Norway

${ }^{3}$ Laboratory of Clinical Biochemistry and Metabolism, Department of General Pediatrics, Adolescent Medicine and Neonatology, Faculty of Medicine, Medical Center, University of Freiburg, Mathildenstraße 1, 79106 Freiburg, Germany ${ }^{4}$ Ludwig Institute for Cancer Research Brussels, 1200 Brussels, Belgium

5 Université Catholique de Louvain and de Duve Institute, 1200 Brussels, Belgium

${ }^{6}$ Department of Molecular Cell Biology, Institute for Cancer Research, Oslo University Hospital, 0424 Oslo, Norway

${ }^{7}$ Department of Pathology, Oslo University Hospital, 0424 Oslo, Norway

${ }^{8}$ Department of Ophthalmology, Cliniques Universitaires Saint-Luc, UCL, 1200 Brussels, Belgium ${ }^{9}$ Department for Cancer Immunology, Institute for Cancer Research, Oslo University Hospital, 0424 Oslo, Norway

${ }^{10}$ Institute of Clinical Medicine, University of Oslo, 0318 Oslo, Norway

11Department of

Haematology, Oslo University Hospital, 0424 Oslo, Norway

12Lead Contact

*Correspondence: judith.staerk@ncmm.uio.no https://doi.org/10.1016/j.isci. 2020.101154 
A
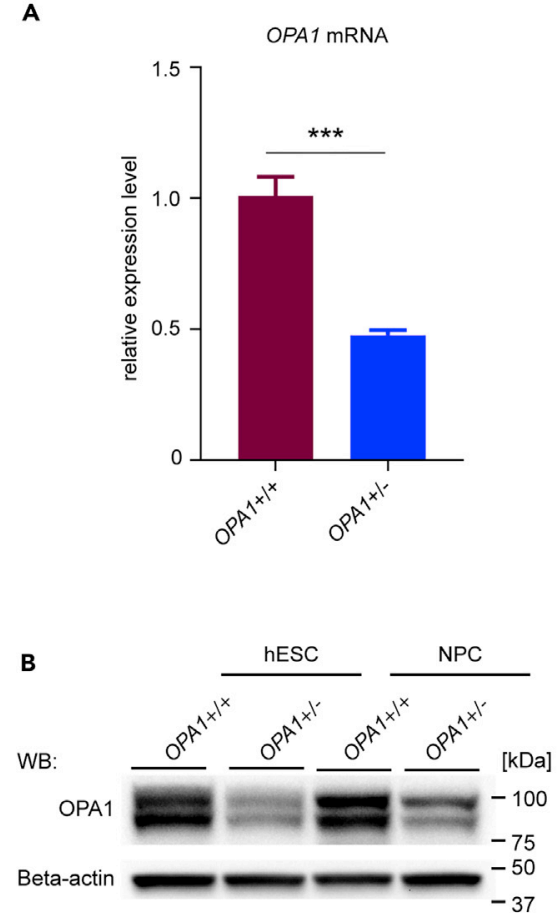

C

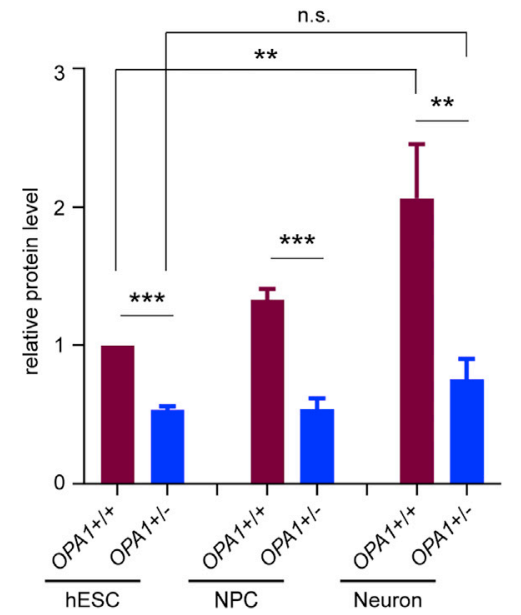

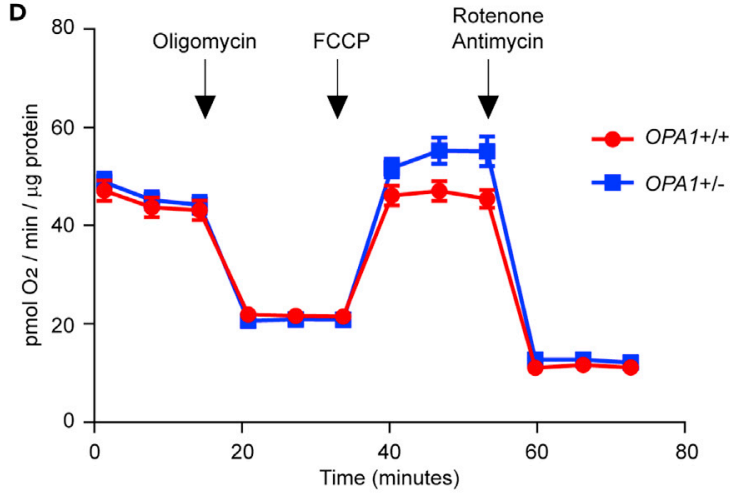
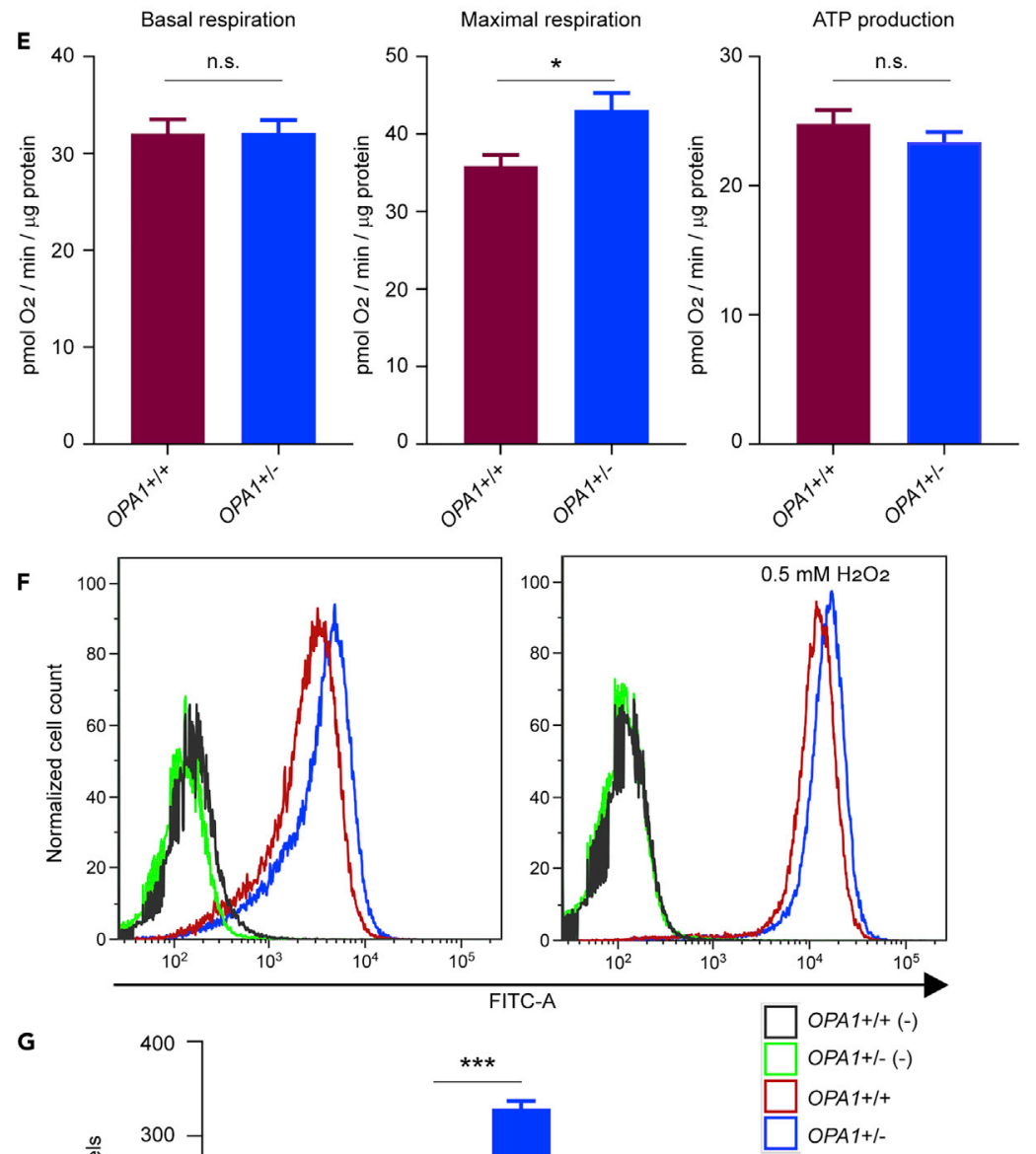

$\mathbf{G}$

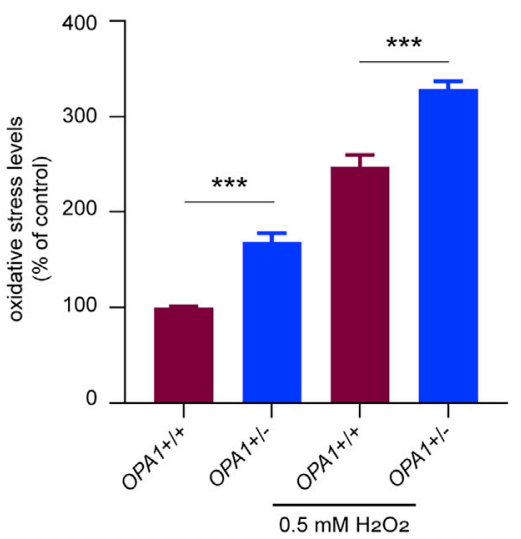


Figure 1. OPA1 Haploinsufficiency Induces Oxidative Stress in hESCs

(A) OPA1 mRNA expression levels in hESCs determined by qRT-PCR analysis.

(B) Representative immunoblotting images showing OPA1 protein levels in hESCs, week $3 \mathrm{NPCs}$, and neurons. Beta-actin was used as loading control.

(C) Densitometric analysis of immunoblots in OPA $1+/+$ and OPA1+/- cells. Mean $\pm \mathrm{SEM}, \mathrm{N}=4$ independent experiments.

(D) Oxygen consumption rate changes under mitochondrial stress in OPA1+/+ and OPA1+/- hESCs. Oligomycin, FCCP, antimycin, and rotenone were sequentially applied. Mean $\pm \mathrm{SEM}, \mathrm{N}=2$ independent experiments.

(E) Basal respiration rates, maximum respiration rates, and ATP production calculated in OPA $1+/+$ and OPA $1+/-h E S C s$. Mean $\pm S E M, N=2$ independent experiments were performed and $N \geq 9$ technical replicates were analyzed for each genotype. Student's $t$ test was used to analyze the difference between two groups. n.s. not significant. ${ }^{\star} p<0.05$.

(F) Representative flow cytometry analysis of OPA1+/+ and OPA1+/- hESCs labeled with the hydrogen peroxide-sensitive oxidative stress indicator CMH2DCFDA. Unlabeled hESCs (-) were used as negative control. hESCs that were incubated with $0.5 \mathrm{mM} \mathrm{H}_{2} \mathrm{O}_{2}$ were used as positive control.

(G) Quantification of flow cytometry measurements in OPA $1+/+$ and OPA $1+/-\mathrm{hESCs}$. Arithmetic mean values were normalized to the values of labeled OPA $1+/+$ hESCS.

Mean \pm SEM, $N \geq 3$ independent experiments and $N=2$ technical replicates. Student's t test was used to analyze difference between two groups. $n . s$. not significant. ${ }^{* \star} p<0.01,{ }^{* \star *} p<0.001$. See also Figures S1, S2, and S4.

(NPCs) and forebrain neurons. Although we were able to generate NPCs and glutamatergic neurons, OPA1 haploinsufficiency interfered with GABAergic interneuron formation. We then explored the molecular changes associated with the observed altered neural cell specification and identified a novel function for OPA1.

\section{RESULTS}

\section{OPA1 Haploinsufficiency Induces Oxidative Stress in hESCs}

To study the role of OPA1 during neural development, we used human embryonic stem cells (hESCs) and the CRISPR-Cas9 gene editing technology and deleted a stretch of nucleotides, which induces a frameshift and a premature stop codon in the second exon of the OPA1 transcript (Figure S1A). Because the majority of human disorders linked to OPA1 are caused by heterozygous mutations, we targeted one allele only. We found a 50\% reduction in OPA1 mRNA transcript levels in heterozygous OPA1+/compared with OPA1+/+hESCs, confirming that only one OPA1 allele was transcribed (Figure 1A). The reduction in mRNA expression levels correlated with a 50\% reduction in OPA1 protein levels in hESCs, indicating a non-sense-mediated RNA decay in OPA1+/- cells (Figures 1B and 1C). Expression levels of OCT4 and NANOG were comparable between OPA1+/ - and parental hESCs, as shown by immunostaining and immunoblotting (Figures S1B and S1C). Moreover, hESC proliferation rates, assessed by automated live-cell analysis system, were similar between the two genotypes (Figure S1D). To further analyze cell cycle dynamics in OPA1+/- hESCs, we performed flow cytometry (fluorescence-activated cell sorting [FACS]) analysis of hESCs labeled with the fluorescent DNA-intercalating dye propidium iodide (PI) and Ki-67 antibody. The ratio of cells in G0/G1, S, and G2/M phases (Figures S1E and S1F), as well as Ki-67 labeling (Figure S1G), were similar between OPA1+/+ and OPA1+/- hESC, indicating that OPA1 haploinsufficiency does not affect pluripotency or proliferation rates in hESCs. Using transmission electron microscopy (TEM), we then assessed mitochondrial morphology and distribution in OPA 1+/hESCs. The TEM images showed no obvious differences in mitochondrial morphology between OPA $1+1+$ and OPA1+/- hESCs (Figure S2A). Next, we measured mitochondrial length in OPA $1+/+$ and OPA1+/- hESCs and binned the data into categories of intervals. Although this analysis revealed no significant change, we found a trend of more fragmented mitochondria in OPA $1+/-$ hESCs (Figure S2B). We therefore determined the circumference of mitochondria, and found a small, but significant decrease in circumference in OPA1+/- compared with OPA1+/+ hESCs (Figure S2C). In addition, we found a significant increase in the intercristae distance in OPA1+/- compared with OPA1+/+ hESCs (Figures S2D and S2E). Mitochondria-targeted GFP (mito-GFP)-transduced hESCs also demonstrated that there was no obvious difference in mitochondrial shape or size among the two genotypes (Figure S2F). The mitochondrial DNA content, as analyzed by copy number of the mitochondrial genes NADH-ubiquinone oxidoreductase chain 1 (MT-ND1) and MT-ND4, was similar between OPA1+/+ and OPA1+/hESCs (Figure S2G). Using mitochondrial respiration assays, we found similar ATP production and basal respiration rates (Figures $1 \mathrm{D}$ and $1 \mathrm{E}$ ) and an increase in maximal respiration in OPA1+/- compared with OPA1+/+ hESCs (Figures $1 \mathrm{D}$ and 1E). Next, we analyzed whether reactive oxygen species (ROS) were altered in OPA1+/- hESCs. Flow cytometry analysis using the hydrogen peroxide-sensitive ROS indicator CM-H2DCFDA revealed significantly increased ROS levels in OPA1+/- compared with OPA1+/+ hESCs (Figures $1 \mathrm{~F}$ and $1 \mathrm{G}$ ). Taken together, our results reveal subtle changes in the mitochondrial morphology and increased levels of ROS in OPA1 haploinsufficient hESCs. 
A

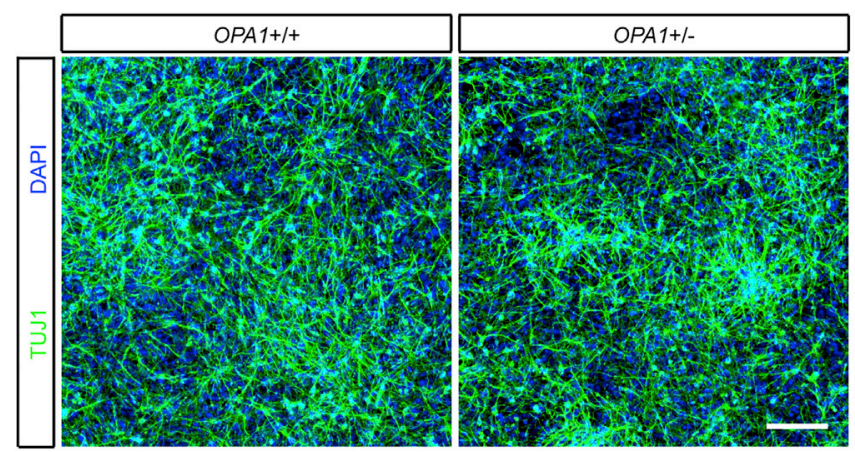

B
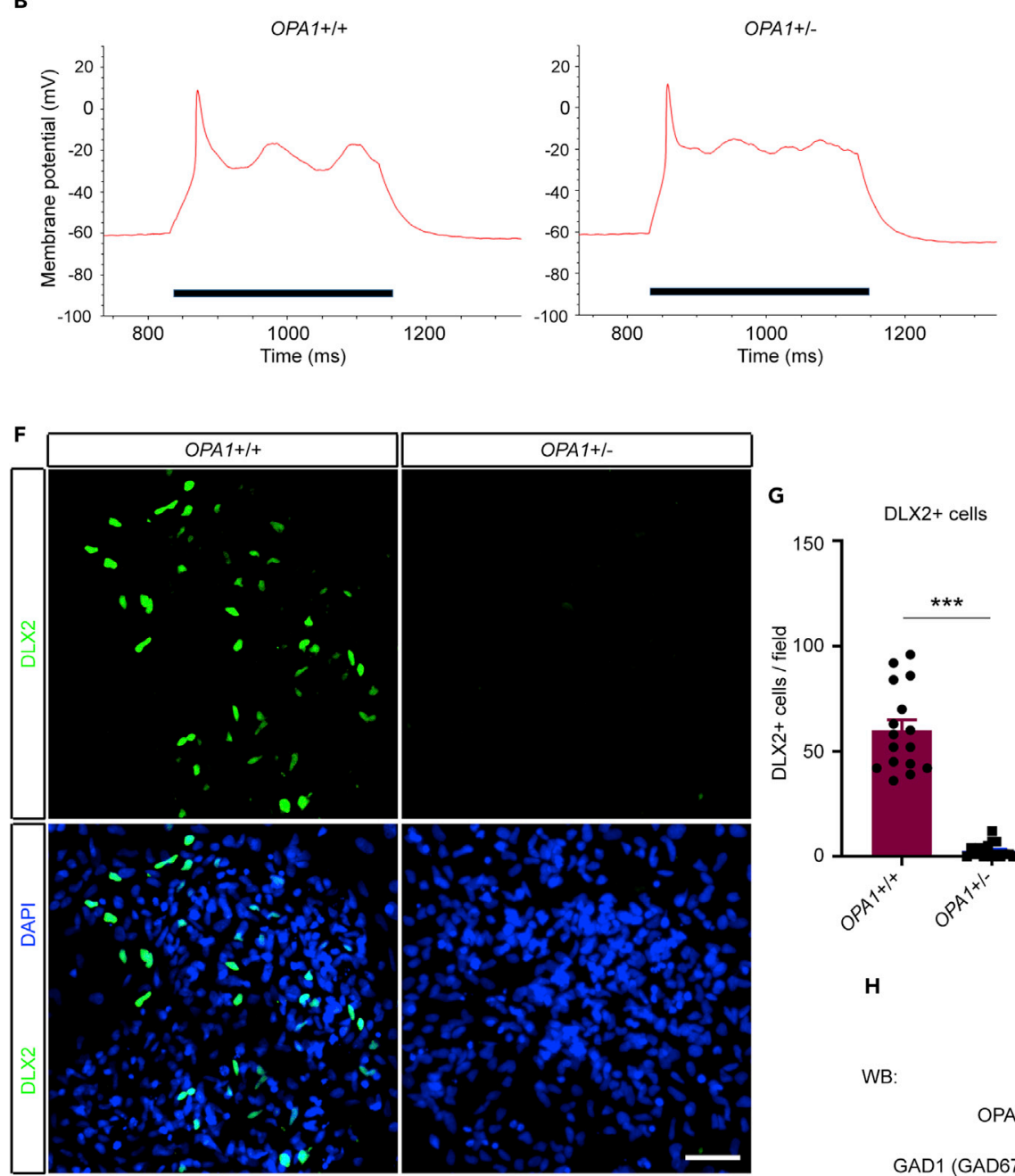

C
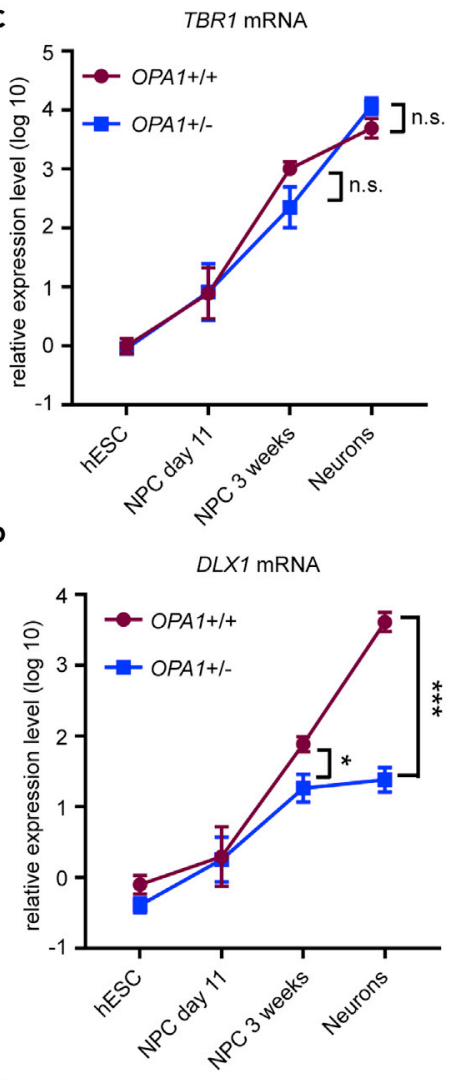

E

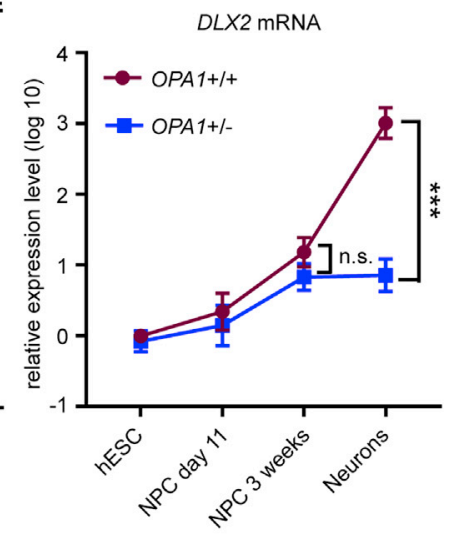

H

WB:
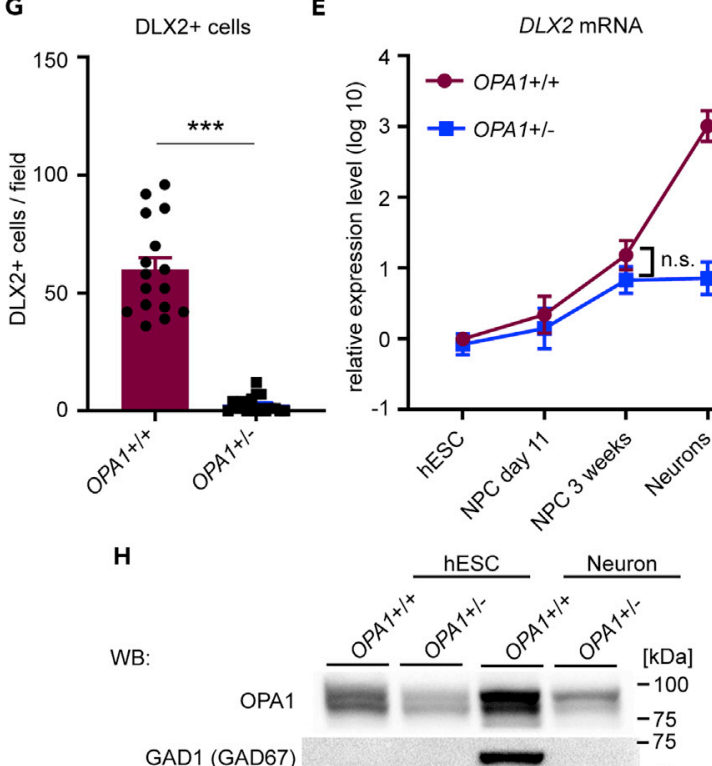

GAD1 (GAD67)

DNM1L

DLX2

$\beta$-Tubulin III (TUJ1)

Beta-actin

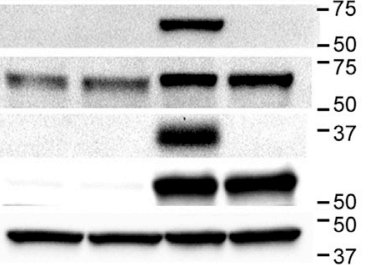


Figure 2. OPA1 Heterozygous NPCs Fail to Generate DLX1/2+ Neurons

(A) Beta-tubulin III (TUJ1) staining of neurons. Scale bar, $100 \mu \mathrm{m}$.

(B) Representative traces of patch-clamped neurons. Action potentials were elicited in OPA1+/+ and OPA1+/-neurons upon depolarizing current injection. Black bar indicates time of depolarizing current injection.

(C-E) (C) TBR1, (D) DLX1, and (E) DLX2 mRNA levels determined by qRT-PCR analyses in OPA1+/+ and OPA1+/- hESCs, NPCs, and neurons. Mean \pm SEM, $\mathrm{N} \geq 3$ independent experiments for each genotype and cell type.

(F) Immunostaining images of OPA1+/+ and OPA1+/- neurons. DAPI was used to stain nuclei. Scale bar, $100 \mu \mathrm{m}$

(G) Quantification of DLX2+ neurons. Mean \pm SEM, $N=3$ independent experiments.

(H) Immunoblotting showing OPA1, DNM1L, GAD1/GAD67, DLX2, and beta-tubulin III protein expression in OPA1+/+ and OPA1+/- hESCs and neurons. Beta-actin was used as loading control.

Student's t test was used to analyze difference between two groups. n.s. not significant. ${ }^{\star} p<0.05$, ${ }^{\star \star *} p<0.001$. See also Figures S3 and S4.

\section{OPA1 Expression Is Upregulated during Neural Differentiation}

Next, we investigated how OPA1 heterozygosity affects neural differentiation. Using the dual SMAD inhibition protocol (Chambers et al., 2009; Shi et al., 2012), hESCs were differentiated into neuroepithelial progenitors and NPCs (Figure S3A). On day 11 of the differentiation protocol, neural commitment was evident by morphological transformation and expression of the neural stem cell marker PAX6 (day 11 NPCs, Figure S3B). After passaging and further differentiation, PAX6-positive NPCs reorganized themselves into neural rosette structures (week 3 NPCs, Figure S3C) before being differentiated into beta-tubulin III-positive neurons (Figure S3D). To assess the neurogenic ability of hESCs, we first compared rosette formation of NPCs. Immunostainings showed that OPA1+/- NPCs formed tight junction protein Zonula-occludens-1 (ZO-1)-positive rosette structures with comparable efficiency as OPA1+/+ cells (Figure S3E). As the self-organization of neural rosettes is reminiscent of neural tube formation from neuroepithelium (Deglincerti et al., 2016), these results reveal that early neural development is not affected by OPA1 haploinsufficiency.

Quantification of OPA1 transcript levels by qRT-PCR showed a $25 \%$ increase of total OPA1 mRNA levels during neuronal differentiation (Figure S4A). OPA1 is alternatively spliced in different tissues. Of these splice variants, OPA1 transcript variant 1 (NM_015560), which lacks exons 4b and 5b (Figure S1A), is the most abundantly expressed isoform in the mouse brain and retina (Delettre et al., 2001; Akepati et al., 2008). In line with this, we found that OPA1 isoform 1 levels markedly increased in our neuronal cultures (Figure S4B). Moreover, we found that isoform 1 became the predominant splice form during neural differentiation. In hESCs, the ratio of transcript variant 1 to total OPA1 mRNA levels was approximately 1:1, whereas in neurons the majority of OPA 1 transcripts were isoform 1 (Figure S4C). Immunoblotting experiments showed a $25 \%$ and 2 -fold increase of OPA 1 protein in OPA $1+/+$ NPCs and neurons when compared with hESCs, respectively (Figures 1B, 1C, and S4D).

\section{OPA1 Heterozygous NPCs Fail to Generate DLX1/2+ Neurons}

To investigate the role of OPA1 during differentiation and neuronal function further, we analyzed neurons derived from OPA 1+/+ and OPA1+/- NPCs. Immunostaining experiments showed that pan-neuronal marker beta-tubulin III-positive neurons could be efficiently generated from both genotypes, indicating that OPA1 haploinsufficiency is not affecting overall neuronal development (Figure 2A). Patch-clamp experiments demonstrated that OPA1 haploinsufficiency did not interfere with the ability of neurons to fire action potentials in response to current stimuli (Figure $2 \mathrm{~B}$ ). To determine neuronal lineage specification, we selected T-box brain 1 (TBR1) as a marker for glutamatergic neurons (Hevner et al., 2001), and DLX1 and DLX2 as they are important for specification of RGCs and GABAergic neurons (Anderson et al., 1997; Letinic et al., 2002; de Melo et al., 2005). As expected, TBR1, DLX1, and DLX2 mRNA expression levels increased during the neuronal differentiation of OPA1+/+ hESCs (Figures 2C-2E), demonstrating that our neuronal cultures included cell types representing forebrain development. During neuronal differentiation of OPA1+/- hESCs, we found that TBR1 was expressed at similar levels as in OPA1+/+ cells (Figure 2C). However, OPA1+/- cells notably failed to induce mRNA expression of DLX1 and DLX2 during neuronal maturation (Figures $2 \mathrm{D}$ and $2 \mathrm{E})$. Using immunostaining, we showed that there were significantly less DLX2 immunoreactive cells in OPA1+/- compared with OPA1+/+ neuronal cultures (Figures $2 \mathrm{~F}$ and $2 \mathrm{G}$ ). The absence of DLX2 protein in lysates of OPA1+/- but not OPA1+/+ neurons was further confirmed using immunoblotting (Figure $2 \mathrm{H}$ ). Neurons derived from both genotypes expressed comparable levels of beta-tubulin III protein (Figure $2 \mathrm{H}$ ).

\section{OPA1 Haploinsufficient NPCs Do Not Give Rise to GABAergic Interneurons}

DLX1 and DLX2 are transcription factors (TFs) important for GABAergic interneuron and RGC development (Letinic et al., 2002; Anderson et al., 1997; de Melo et al., 2005). As their downregulation indicated 
A

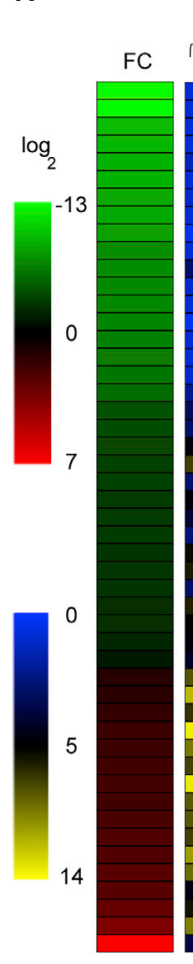

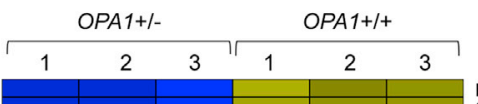
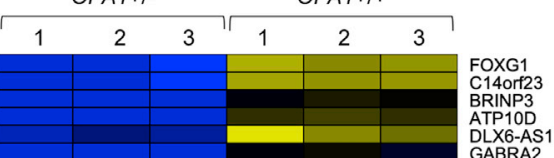

FOXG1

BRINP3
ATP10D

DLX6-AS

GABRA2

GABRA4

DLX5
NEUROD6

DLX1

LINC013

CHL1

DLX2

GABRG1

KLITRK2

SLITRK

GAD2

MOXD1

OPRM1
SLITRK4

FZD8

SLC32A1

MPPED2

GABRA5

GAD1

SCN1A

DOK5

NR2F2

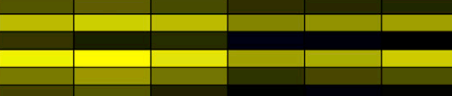

NR2F1

NRP1

PPP1R17

NEFM
PCSK1

NEUROD

ARL4A

LOC 440
SIM1

PRDM8

MAB21L2

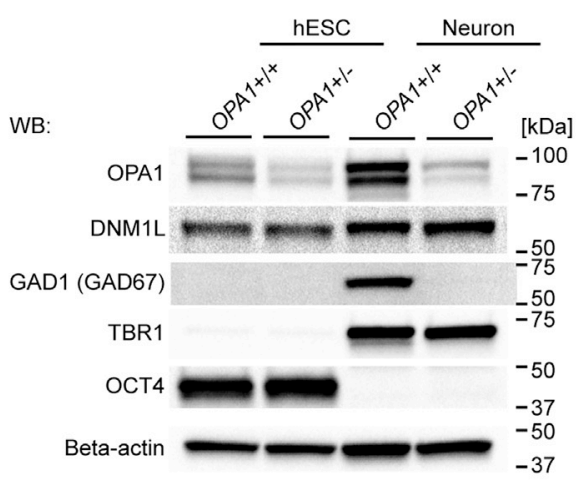

C GAD1 / GAD67

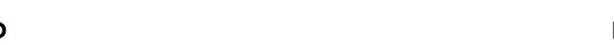

WB:

GAD2 (GAD65)

$\beta$-Tubulin III (TUJ1)

Beta-actin

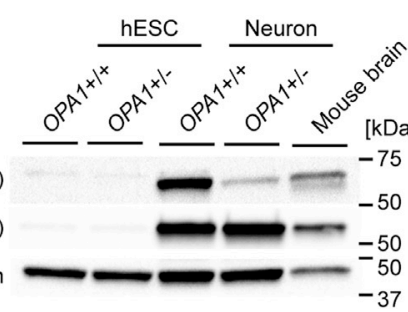

E

GAD2 / GAD65

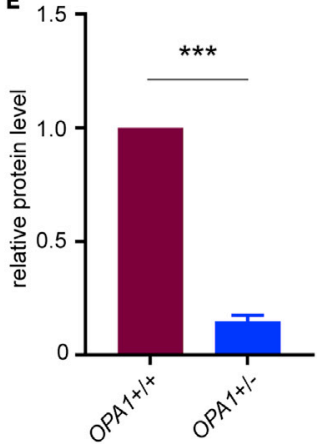

Figure 3. OPA1 Haploinsufficient NPCs Do Not Give Rise to GABAergic Interneurons

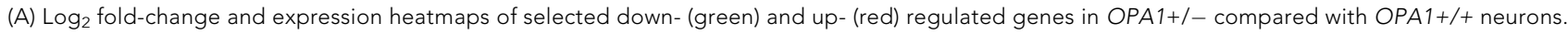
$\mathrm{FC}=$ fold-change. Expression intensities are displayed from blue (low expression) to yellow (high expression).

(B) Immunoblotting showing OPA1, DNM1L, TBR1, and GAD1/GAD67 protein expression in OPA1+/+ and OPA1+/ $-\mathrm{hESCs}$ and neurons. OCT4 was used as pluripotency marker; beta-actin was used as loading control.

(C) Densitometric analysis of GAD1 protein levels in OPA1+/+ and OPA1+/- neurons. Mean \pm SEM, N = 2 independent experiments.

(D) Immunoblotting showing GAD2/GAD65 and beta-tubulin III protein expression in OPA1+/+ and OPA1+/-hESCs and neurons.

(E) Densitometric analysis of GAD2 protein levels in OPA1+/+ and OPA1+/- neurons. Mean \pm SEM, $N=2$ independent experiments.

Student's $t$ test was used to analyze difference between two groups. ${ }^{\star \star \star} p<0.001$. See also Figures S3 and S4, and Table S1.

an altered neuronal function and differentiation potential when OPA1 was reduced, we performed RNA sequencing (RNA-seq) in 7-week-old neurons. We found several genes encoding for TFs with known function in neurogenesis differentially regulated in OPA1+/- neurons (Table S1). For instance, FOXG1, DLX1, $D L X 2, D L X 5$, and DLX6 were genes highly downregulated in OPA1+/- compared with OPA1+/+ neurons (Figure 3A; Table S1). Moreover, expression of SFRP1 and SEMA5A, which are known to control RGC development and axonal growth, as well as CTIP2/BCL11B and NEUROD6, which are TFs that are highly expressed in the developing brain and retinal cells (Cherry et al., 2011), were also significantly downregulated. Other genes downregulated included SLITRK2, SLITRK4, and SLITRK6, which are plasma membrane proteins expressed in the retina, and several GABA receptor subunits (GABRA2, GABRA4, GABRB1, and GABRG1). Among the upregulated genes in OPA1+/- neurons we found NR2F1, NR2F2, and NEUROD4, which are TFs known to regulate cell fate specification in the retina (Figure 3A).

The absence of DLX1/2 expression in OPA1+/- neurons prompted us to further investigate GABAergic neuronal markers. Glutamate decarboxylase 1 (GAD1/GAD67) and 2 (GAD2/GAD65), which catalyze the production of GABA from L-glutamic acid, were expressed in OPA1+/+ neurons, whereas they were absent or markedly reduced in OPA1+/- neurons (Figures 3B-3E and S4E). TBR1 and beta-tubulin III were expressed at comparable levels (Figures $3 \mathrm{~B}$ and $3 \mathrm{D}$ ). Overall, our transcriptional analysis demonstrates that reduced OPA1 protein levels alter the expression of genes that are important for GABAergic interneuron formation and retinal development. 

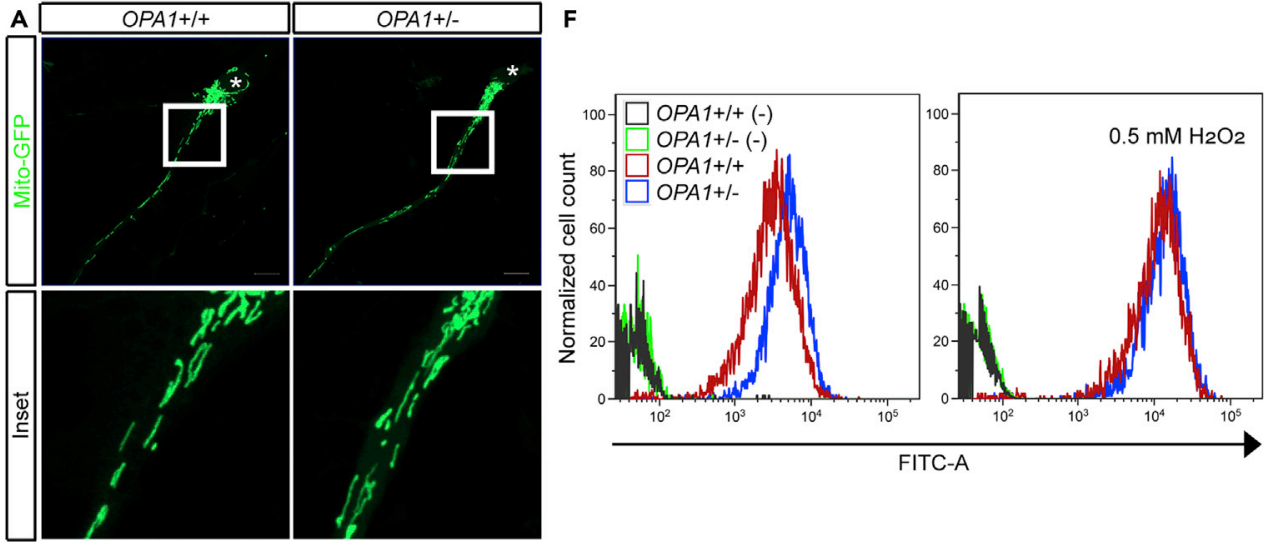

G

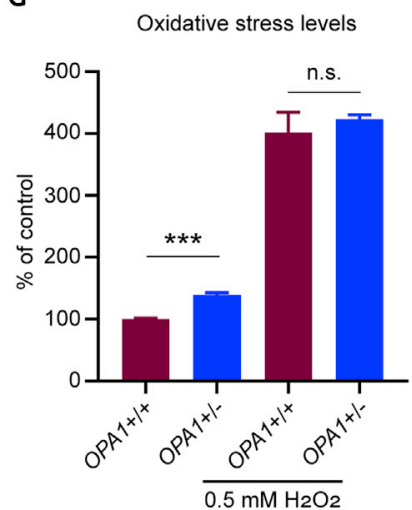

B

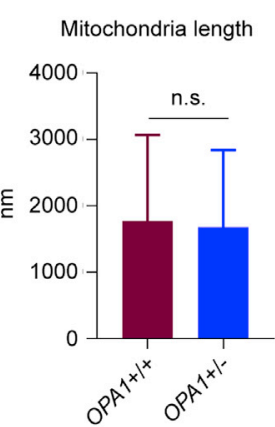

c
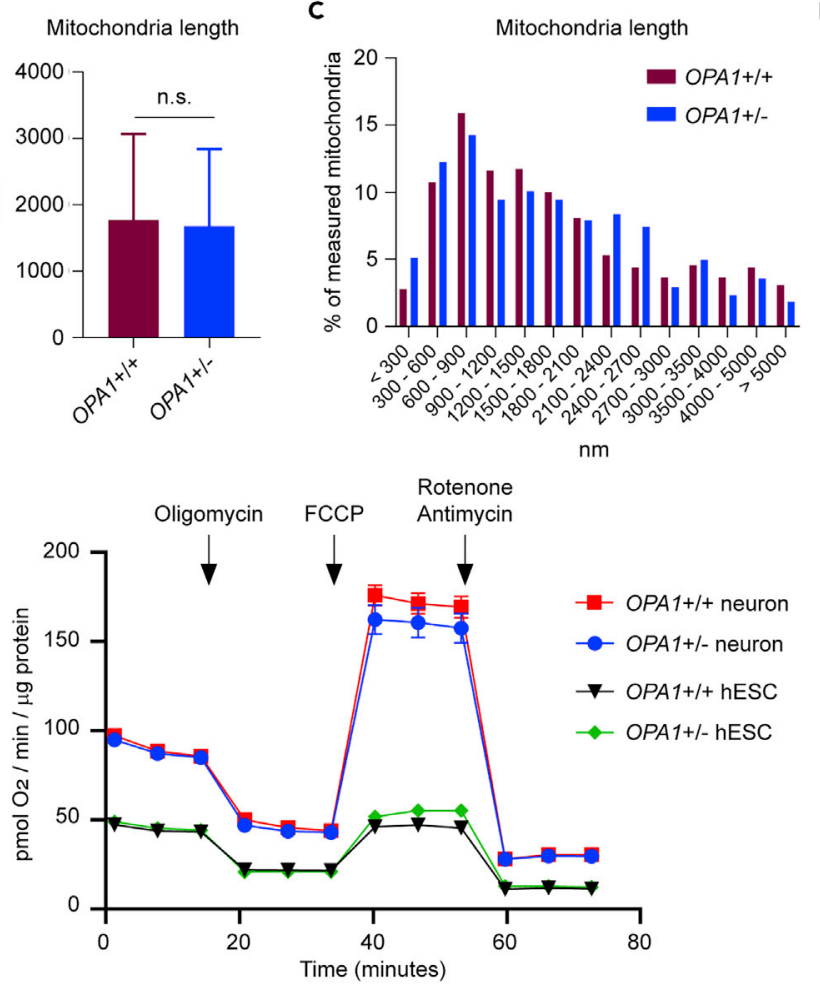

E Basal respiration

Maximal respiration ATP production
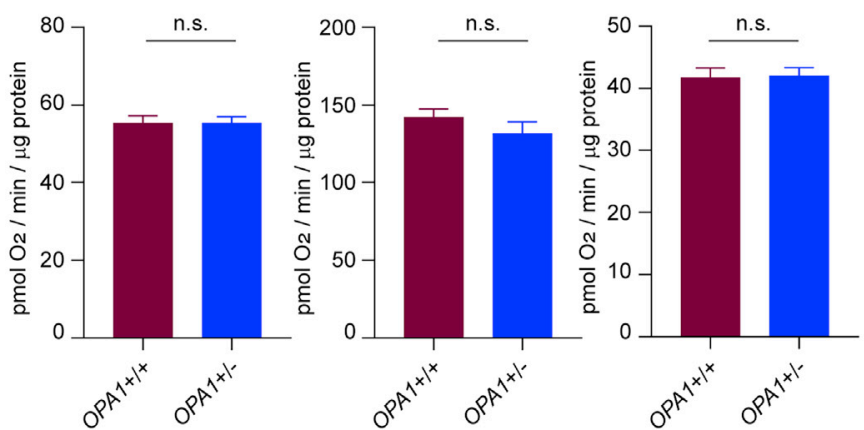
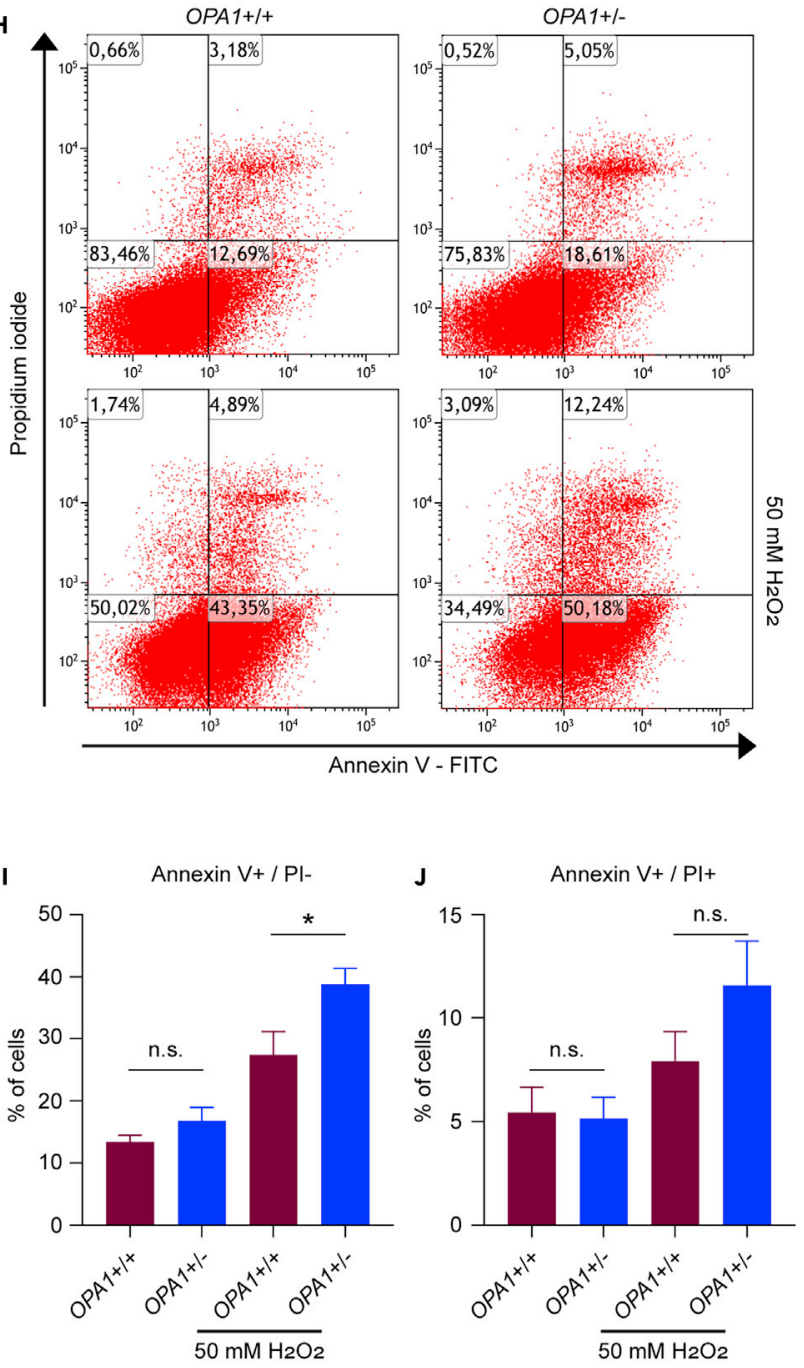
Figure 4. OPA1 Haploinsufficiency Induces Oxidative Stress and Apoptosis in Neurons

(A) Confocal microscopic images of mito-GFP-transduced OPA1+/+ and OPA1+/- neurons. * denotes nucleus. Scale bar, $10 \mu \mathrm{m}$.

(B) Quantification of mitochondria length in OPA1+/+ and OPA1+/- neurons. $\mathrm{N}=680$ mitochondria for OPA1+/+ and N = 645 mitochondria for OPA1+/were measured.

(C) Mitochondrial length was measured and binned into categories of length intervals.

(D) Oxygen consumption rate changes under mitochondrial stress in OPA1+/+ and OPA1+/- neurons. Oligomycin, FCCP, antimycin, and rotenone were sequentially applied. Mean $\pm \mathrm{SEM}, \mathrm{N}=2$ independent experiments.

(E) Basal respiration rates, maximum respiration rates, and ATP production calculated in OPA $1+/+$ and OPA $1+/-$ neurons. Mean $\pm S E M, N=2$ independent experiments were performed and $N \geq 40$ technical replicates were analyzed for each genotype.

(F) Representative flow cytometry analysis of OPA1+/+ and OPA1+/- neurons labeled with the hydrogen peroxide-sensitive oxidative stress indicator CMH2DCFDA. Unlabeled neurons (-) were used as negative control. Neurons that were incubated with $0.5 \mathrm{mM} \mathrm{H}_{2} \mathrm{O}_{2}$ were used as positive control.

(G) Quantification of flow cytometry measurements in OPA1+/+ and OPA1+/- neurons. Arithmetic mean values were normalized to the values of labeled OPA $1+/+$ neurons. Mean $\pm S E M, N \geq 3$ independent experiments and $N=2$ technical replicates.

(H) Representative flow cytometry analysis of live OPA1+/+ and OPA1+/- neurons labeled with the early apoptosis indicator annexin $V$ and late-apoptosis indicator propidium iodide (PI). Neurons were incubated with $50 \mathrm{mM} \mathrm{H}_{2} \mathrm{O}_{2}$ to assess sensitivity to apoptotic stimuli.

(I) Quantification of early apoptotic annexin V+/PI- neurons. Percentage of labeled cells in total gated cells is shown.

(J) Quantification of late-apoptotic Annexin V+/PI+ neurons. Percentage of labeled cells in total gated cells is shown.

Mean $\pm \mathrm{SEM}, \mathrm{N}=4$ independent experiments and $\mathrm{N}=2$ technical replicates. Student's $\mathrm{t}$ test was used to analyze difference between two groups. $\mathrm{n} . \mathrm{s}$. not significant. ${ }^{*} p<0.05,{ }^{* * \star} p<0.001$. See also Figures S3 and S4.

We imaged mito-GFP-transduced neurons to assess mitochondrial morphology. Confocal microscopy analysis demonstrated that mitochondrial morphology and distribution were similar in OPA1+/- and OPA1+/+ neurons (Figure 4A). Quantification of mitochondrial length and binning of measurements into categories of intervals showed no evident differences in mitochondrial distribution (Figures $4 \mathrm{~B}$ and $4 \mathrm{C}$ ). Next, we analyzed energy metabolism in OPA1+/- and OPA1+/+ neuronal cultures using mitochondrial respiration assays. Our data revealed that neurons exhibited an increased mitochondrial respiration compared with hESCs (Figure 4D). However, basal respiration, maximal respiration, and ATP production rates were similar in OPA $1+/-$ and OPA1+/+ neurons (Figure 4E), suggesting that OPA1 haploinsufficiency does not affect mitochondrial respiration in neurons. We then investigated oxidative stress levels in neuronal cultures. Flow cytometry analysis showed significantly increased ROS levels in OPA $1+1-$ compared with OPA1+/+ neurons (Figures 4F and 4G). FACS analysis of neurons labeled with the early apoptosis indicator Annexin $\mathrm{V}$ and late apoptosis indicator PI (Figure 4H) demonstrated no differences in apoptosis in neuronal culture conditions (Figures $4 \mathrm{I}$ and $4 \mathrm{~J}$ ). Importantly, when neurons were exposed to stress by pre-treatment with hydrogen peroxide we observed significantly more Annexin $\mathrm{V}+$ cells in OPA $1+/$ - compared with OPA $1+/+$ neurons (Figures $4 \mathrm{I}$ and $4 \mathrm{~J}$ ).

\section{OPA1 Heterozygous NPCs Exhibit a Significantly Altered Transcriptional Signature}

Next, we addressed whether the impaired formation of DLX1/2+ neurons in OPA1+/- cultures could be explained by an altered transcriptional circuitry at the NPC level. Successful formation of day 11 NPCs was verified by the loss of the pluripotency markers OCT4 and NANOG, and the gain of the neural stem cell marker PAX6 (Figure S5A). We then performed RNA-seq analysis in OPA1+/+ and OPA1+/- NPCs after 11 days and 3 weeks of differentiation to elucidate potential transcriptional changes associated with OPA 1 haploinsufficiency. Principal-component analysis was used to assess and visualize the neural differentiation trajectory (Figure 5A). When we analyzed differentially expressed genes (DEGs) at day 11 OPA1+/- versus OPA1+/+ NPCs, we found FOXG1 already highly downregulated at this early NPC stage (Figure 5B and Table S2). Another gene we found highly downregulated encodes for TAF9B (Figure 5B and Table S2), a protein that is part of the neuronal core promoter complex and known to regulate the induction of specific neuronal genes (Herrera et al., 2014). Moreover, we found SLITRK2, a transmembrane protein expressed in proliferative neuroblastic layer of the retina (Beaubien and Cloutier, 2009), to be significantly downregulated in OPA1+/- NPCs (Figure 5B and Table S2). In addition, GABRA2, the gene coding for GABRA receptor alpha 2; ATP10D, a putative phospholipid transporting ATPase; and several neural cell adhesion genes, including NCAM2, PCDHGA3, and PCDHGA6, were also downregulated (Figure 5B and Table S2). Significantly upregulated genes in day 11 OPA1+/- versus OPA1+/+ NPCs included genes coding for neural guidance proteins, such as SEMA3E, SLIT3, Ephrin type-A receptor 1 (EPHA1), and its ligand ephrin A1 (EFNA1) (Figure 5B and Table S2).

DEG analysis of OPA1+/- compared with OPA1+/+ NPCs in week 3 revealed that FOXG1, ATP10D, GABRA2, SLITRK2, ZNF280D, and TAF9B also remained highly downregulated at this later stage of differentiation (Figure S5B and Table S3). In addition, several genes encoding for TFs involved in primary 
A

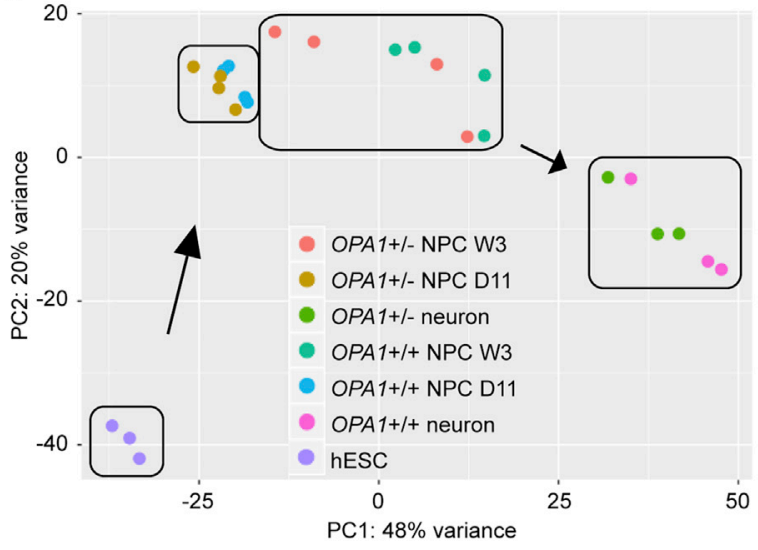

B

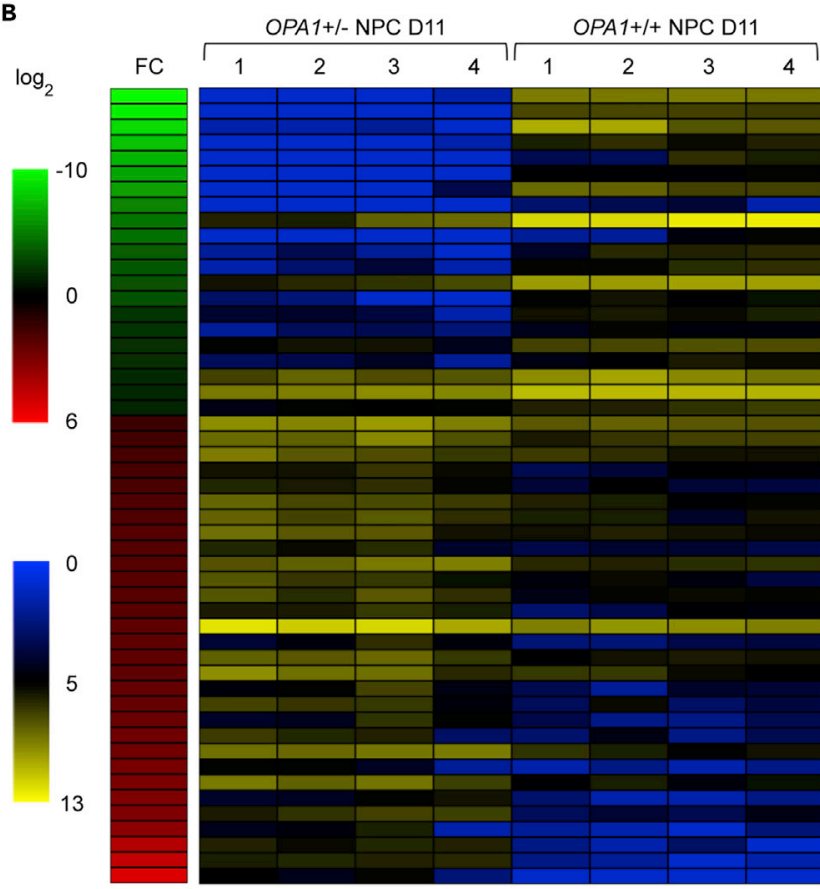

C

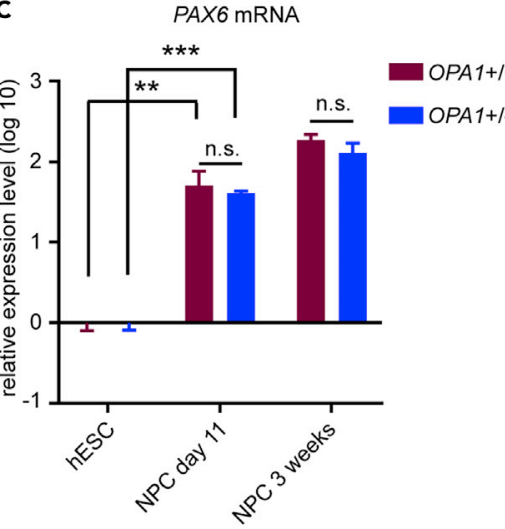

FOXG1 mRNA

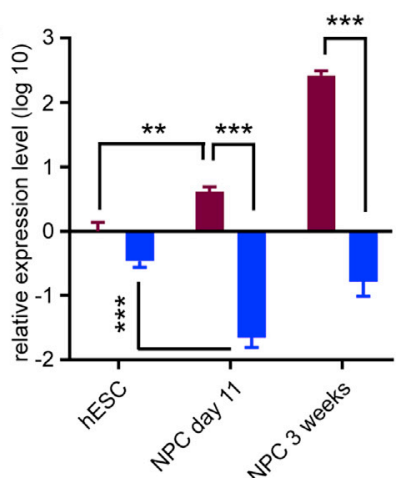

E WB:

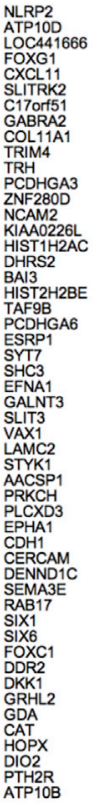

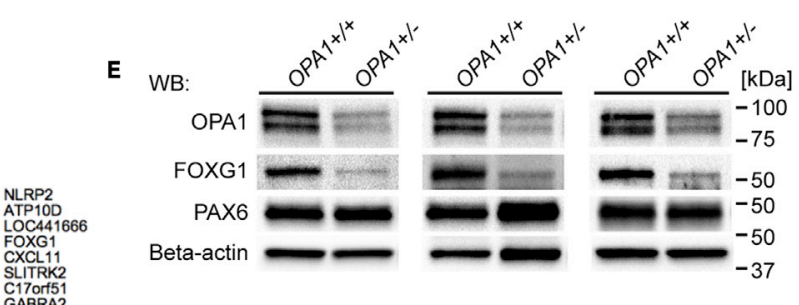

Downregulated genes
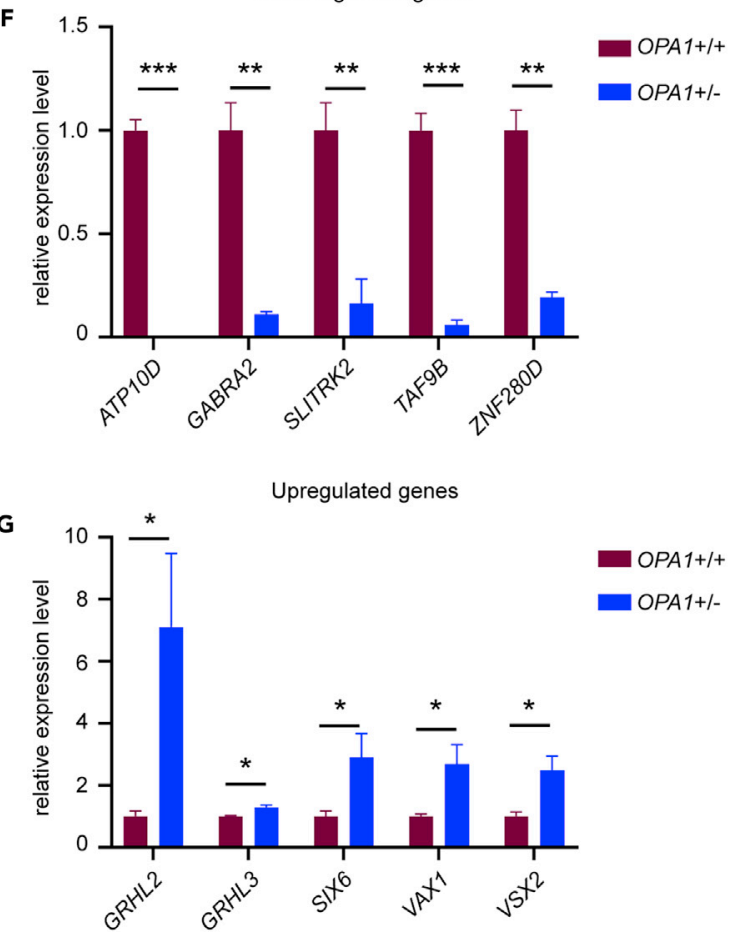

Figure 5. OPA1 Haploinsufficiency Significantly Alters the Transcriptional Circuitry in NPCs

(A) Principal-component analysis of hESCs, day 11 and week 3 NPCs, and neurons.

(B) Log 2 fold-change and expression heatmaps of selected down- (green) and up- (red) regulated genes in OPA1+/- compared with OPA1+/+ day 11 NPCs. $\mathrm{FC}=$ fold-change. Expression intensities are displayed from blue (low expression) to yellow (high expression).

(C and D) (C) PAX6 and (D) FOXG1 mRNA levels in day 11 and week 3 NPCs, normalized to transcript levels in OPA1+/+hESCs.

(E) Immunoblotting showing OPA1, FOXG1, and PAX6 protein expression in NPCs. Beta-actin was used as loading control. N $=3$ independent experiments. (F) ATP10D, GABRA2, SLITRK2, TAF9B, and ZNF280D mRNA expression levels in day 11 NPCs. mRNA expression levels were normalized to transcript levels in OPA1+/+ NPCs. 
Figure 5. Continued

(G) GRHL2, GRHL3, SIX6, VAX1, and VSX2 mRNA expression levels in day 11 NPCs. mRNA expression levels were normalized to transcript levels in OPA1+/+ NPCs.

Mean \pm SEM, $N \geq 4$ independent samples for each genotype and cell type. Student's t test was used to analyze the differences between two groups. n.s. not significant. ${ }^{\star} p<0.05,{ }^{\star \star} p<0.01,{ }^{\star \star *} p<0.001$. See also Figures S3, S5, and S6, and Tables S2 and S3.

neurulation, forebrain development, and retinal development were upregulated in OPA1+/- NPCs. These included SIX6, GRHL2, GRHL3, VAX1, and VSX2 (Figure S5B and Table S3). Overall, our RNA-seq analyses identified significant alterations in the transcriptional circuitry of OPA1+/- NPCs (Figures 5B and S5B, Tables S2 and S3), which can explain the observed altered neuronal cell fate specification.

We then assessed the expression levels of selected genes in more detail. qRT-PCR analysis at three different time points showed continuous increase of PAX6 mRNA expression levels during NPC derivation (Figure 5 C). OPA1+/+ NPCs also showed significant upregulation of FOXG1, starting 11 days after differentiation (Figure 5D), a finding in line with its previously described critical role in early brain development (Xuan et al., 1995). OPA1+/- NPCs expressed similar PAX6 mRNA levels as OPA1+/+ NPCs (Figure 5C), whereas FOXG1 transcription was absent in OPA1+/- cells during all stages of NPC differentiation (Figure 5D). This was also true at the protein level (Figure 5E). In addition, we used qRT-PCR analyses to confirm the downregulation of ATP10, GABRA2, SLITRK2, TAF9B, and ZNF280D (Figure 5F) and upregulation of GRHL2, GRHL3, SIX6, VAX1, and VSX2 mRNA expression in OPA1+/ - compared with OPA1+/+ NPCs (Figure $5 \mathrm{G}$ ). Using a second, independently derived OPA1+/ - hESC clone \#63, we confirmed reduced OPA1 protein (Figure S6A) and OPA1 mRNA expression levels in OPA1+/- compared with OPA1+1+ hESC and NPCs, respectively. Moreover, PAX6 mRNA expression was comparable between genotypes (Figure S6B), whereas FOXG1 and TAF9B mRNA expression levels were also markedly reduced in OPA1+1- \#63 NPCs (Figure S6B).

To investigate whether altered transcriptional regulation affects proliferation or cell survival of OPA1+/NPCs, we performed FACS analysis of NPCs labeled with PI. Figures S5C and S5D showed no differences in cell cycle dynamics in OPA1+/+ and OPA1+/- NPCs. Similarly, Ki-67 expression was also comparable between the two genotypes (Figure S5E). Next, we analyzed the levels of apoptosis in NPCs under normal conditions and upon stimulation with hydrogen peroxide (Figure S5F). We found no significant difference in apoptosis between OPA1+/+ and OPA1+/- NPCs (Figure S5G), whereas pre-treatment of NPCs with hydrogen peroxide increased Annexin $\mathrm{V}+/ \mathrm{PI}+$ late apoptotic cells in OPA1+/- NPCs (Figure S5H), supporting our results that OPA1 haploinsufficiency increases the sensitivity of cells to apoptotic stimuli. Collectively, our data demonstrate that OPA1 is essential for proper transcriptional regulation in NPCs, and that OPA1+/- NPCs exhibit a developmental defect that causes impaired neural subtype specification.

\section{OPA1 Protects against Aberrant DNA Methylation}

Recently, it has been shown that metabolic stress and accumulation of mitochondria affect hematopoietic stem cell fate decisions through altered nuclear DNA methylation (Ho et al., 2017). Our transcriptome analyses showed that several genes important for NPC differentiation, GABAergic interneuron formation, and retinal development such as the pioneer transcription factor FOXG1 were strongly downregulated in OPA1+/NPCs. To address whether this downregulation was caused by epigenetic alterations, we analyzed the CpG content in annotated sequences and found CpG-enriched promoters in genes encoding FOXG1, ATP10D, TAF9B, SLITRK2, GABRA2, and ZNF280D. Figure 6A depicts the location and CpG-enriched regions in the promoter and 5' UTR of FOXG1. Genomic DNA was isolated from NPCs, bisulfite treated, and the FOXG1 5'UTR region was sequenced. We found that all CPG bases within this region were methylated in OPA1+/- cells, whereas this was not the case in OPA1+/+ cells (Figure 6B). This result demonstrates that DNA hypermethylation was the reason for the observed repression of FOXG1 transcription. The amplification of the FOXG1 promoter CpG island was not possible due to the long $\mathrm{CpG}$ stretches, which prevented optimal primer design (Figure 6A). Bisulfite sequencing of the ATP10D locus also revealed a high content of methylated promoter CpG in OPA1+/- but not in OPA1+/+ NPCs (Figures 6C and 6D), further confirming that DNA methylation patterns were altered in OPA1+/- NPCs. Taken together, our results suggest that OPA1 is necessary for NPC transcriptional regulation by preventing aberrant methylation.

Next, we tested whether inhibition of DNA methylation can restore the transcriptional silencing in OPA1+/ - NPCs. Strikingly, incubation of cells with the DNA methyltransferase (DNMT) inhibitor RG108 during 


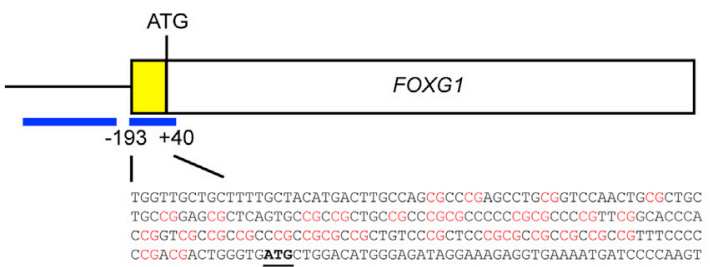

B

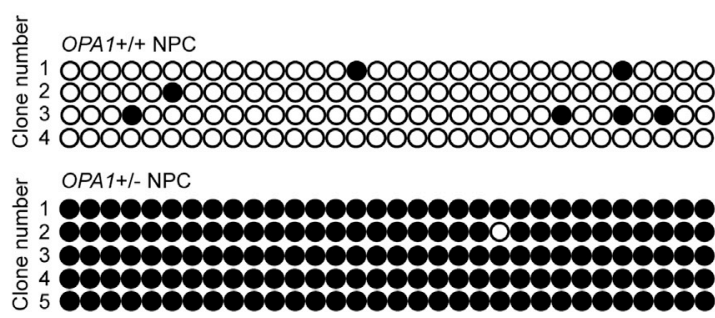

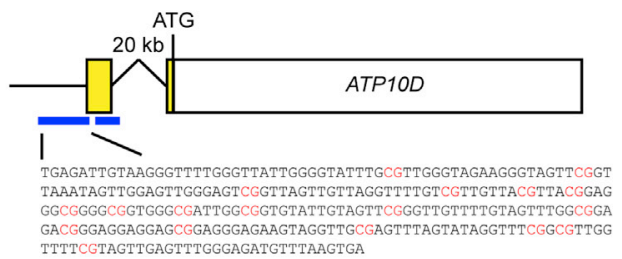

D
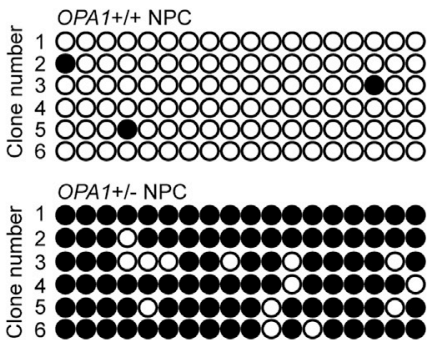

-ketoglutarate
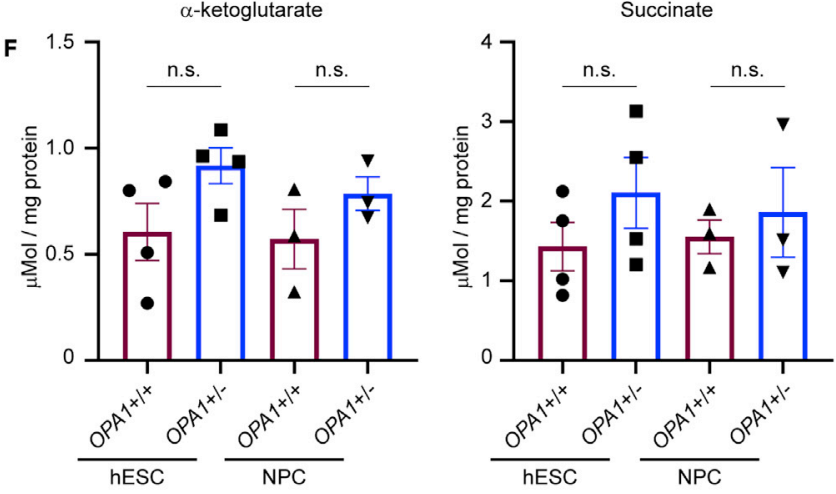

G
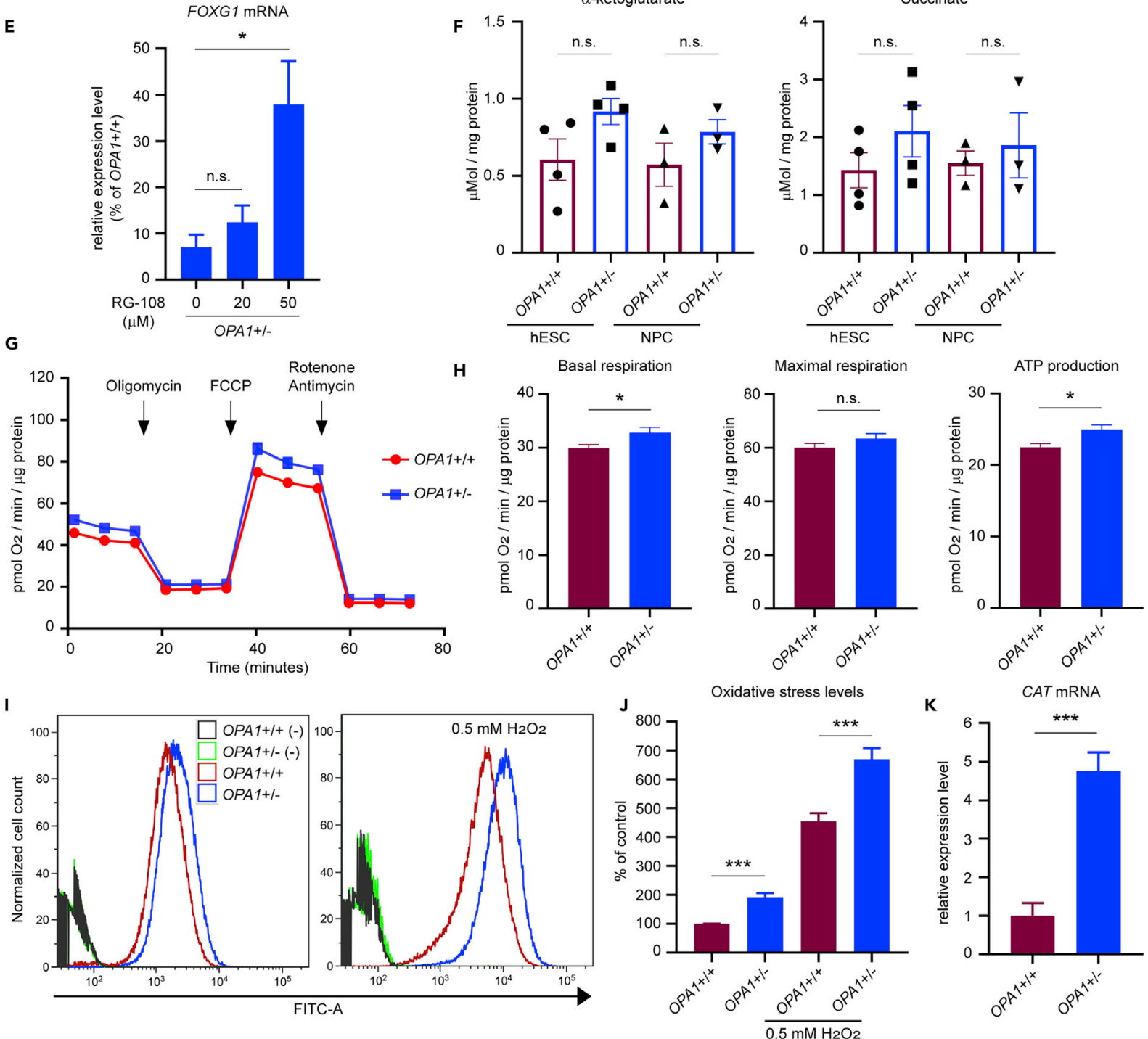
Figure 6. OPA1 Protects against Aberrant DNA Methylation

(A) Location and sequence of CpG-enriched regions in the FOXG1 gene promoter and 5' UTR. ATG is indicated as translation start codon. Numbers denote sequences upstream (-) or downstream (+) of the start codon. The yellow box indicates the $5^{\prime}$ UTR, and blue lines represent CpG enriched regions. CpGs are marked in red.

(B) Bisulfite sequencing of the FOXG1 5'UTR in OPA1+/+ and OPA1+/- NPCs. Empty circles represent unmodified cytosines, and filled circles represent methylated cytosines.

(C) Location and sequence of CPG-enriched regions in the ATP10D gene promoter and 5' UTR. ATG is indicated as translation start codon, and numbers denote sequences upstream (-) or downstream (+) of the start codon. Yellow box indicates the 5' UTR flanked by a 20-kb intron, and blue lines represent CpG-enriched regions. CpGs are marked in red.

(D) Bisulfite sequencing of the ATP10D gene promoter in OPA1+/+ and OPA1+/- NPCs. Empty circles represent unmodified CpG bases, and filled circles represent methylated cytosines.

(E) FOXG1 mRNA levels in day 11 OPA1+/- NPCs treated with different concentrations of DNMT inhibitor RG108. Mean \pm SEM, N $=4$ independent experiments with $\mathrm{N}=2$ technical replicates for each condition. mRNA expression levels were normalized to transcript levels in untreated OPA1+/+ NPCs. (F) Succinate and alpha-ketoglutarate levels measured in OPA1+/+ and OPA1+/- hESCs and day 11 NPCs measured by mass spectrometry analysis. Mean $\pm \mathrm{SEM}, \mathrm{N} \geq 3$ independent samples for each genotype and cell type.

(G) Oxygen consumption rate changes under mitochondrial stress in OPA1+/+ and OPA1+/- NPCs. Oligomycin, FCCP, antimycin, and rotenone were sequentially applied. Mean $\pm \mathrm{SEM}, \mathrm{N}=3$ independent experiments.

(H) Basal respiration rates, maximum respiration rates, and ATP production in OPA $1+/+$ and OPA $1+/-\mathrm{NPCs}$. Mean $\pm \mathrm{SEM}, \mathrm{N}=3$ independent experiments were performed and $\mathrm{N} \geq 40$ technical replicates were analyzed for each genotype.

(I) Representative flow cytometry analysis of OPA1+/+ and OPA1+/- NPCs labeled with the hydrogen peroxide-sensitive oxidative stress indicator CMH2DCFDA. Unlabeled NPCs (-) were used as negative control. NPCs that were incubated with $0.5 \mathrm{mM} \mathrm{H}_{2} \mathrm{O}_{2}$ were used as positive control.

(J) Quantification of flow cytometry measurements in OPA $1+/+$ and OPA1+/- NPCs. Arithmetic mean values were normalized to the values of labeled OPA1+/+ NPCs. Mean \pm SEM, $N \geq 4$ independent experiments and $N=2$ technical replicates.

(K) CAT mRNA expression levels in day 11 OPA1+/+ and OPA1+/- NPCs. mRNA expression levels were normalized to transcript levels in OPA1+/+ NPCs. Mean \pm SEM, $N \geq 3$ independent samples were assessed for each genotype. Student's t test was used to analyze the difference between two groups. n.s. not significant. ${ }^{\star} p<0.05,{ }^{* * *} p<0.001$. See also Figures S6 and S7.

neural differentiation resulted in a partial rescue of FOXG1 expression in OPA1+/- NPCs, demonstrating that the transcriptional silencing caused by OPA1 haploinsufficiency could be rescued by inhibiting DNMTs and preventing DNA methylation (Figure 6E). To determine whether DNA methylation and hydroxymethylation levels were affected on a global level in OPA1+/- NPCs, we used mass spectrometry-based quantification and found similar total methylation $(5 \mathrm{me}(\mathrm{dC}))$ and hydroxymethylation $(5 \mathrm{hm}(\mathrm{dC}))$ levels in OPA1+/+ and OPA1+/- NPCs (Figure S7A). Moreover, immunoblotting for DNMT3A and DNMT3B proteins showed similar expression levels between different genotypes, suggesting that reduced OPA1 levels lead to loci-specific rather than global DNA methylation changes (Figure S7B).

To further explore the potential cause for the observed loci-specific changes in DNA methylation, we analyzed the concentration of the small metabolites S-adenosylmethionine (SAM) and S-adenosylhomocysteine (SAH), which serve as substrates for DNMTs. Using mass spectrometry analysis we demonstrate that the overall SAM/SAH ratio in NPCs of both OPA1+/ - and OPA1+/+ genotypes was significantly higher than in hESCs, indicating an overall higher methylation capacity in NPCs (Figure S7C). However, the overall SAM/SAH ratio was not altered when OPA1 was reduced (Figure S7C). Next, we quantified succinate and alpha-ketoglutarate levels in hESCs and NPCs. Again, there were no significant changes in succinate and alpha-ketoglutarate levels in OPA1+/ - versus OPA1+/+ cells (Figure 6F), indicating that altered availability of these small metabolites is likely not the reason for the observed changes in DNA methylation.

When analyzing the mitochondrial energy metabolism in NPCs, we found a small but significant increase in basal respiration and ATP production levels in OPA1+/- compared with OPA1+/+ NPCs (Figures 6G and $6 \mathrm{H})$. In addition, flow cytometry analysis showed significantly increased ROS levels in OPA1+/ - NPCs (Figures 61 and $6 \mathrm{~J}$ ), further confirming our results in hESC and neurons. In line with the observed increased oxidative stress level in OPA1+/- NPCs, we found catalase (CAT) and FOXC1 mRNA expression levels significantly upregulated in day 11 OPA1+/- NPCs (Figure 5B and Table S2). Catalase is a peroxisomal enzyme protecting cells against oxidative stress, and FOXC1 is a stress-responsive TF that provides resistance to oxidative stress in the eye (Mirzayans et al., 2007). The upregulation of CAT expression was confirmed by qRT-PCR in NPCs derived from two independent OPA1+/- lines (Figures 6K and S6B).

\section{Patient-Derived iPSC Harboring a Pathogenic OPA1 Mutation Exhibit Increased ROS Levels and Downregulation of FOXG1}

Heterozygous mutations in OPA1 are a common cause of ADOA (Delettre et al., 2000; Alexander et al., 2000). To ensure that the changes detected in our genetically engineered hESCs were not caused by 


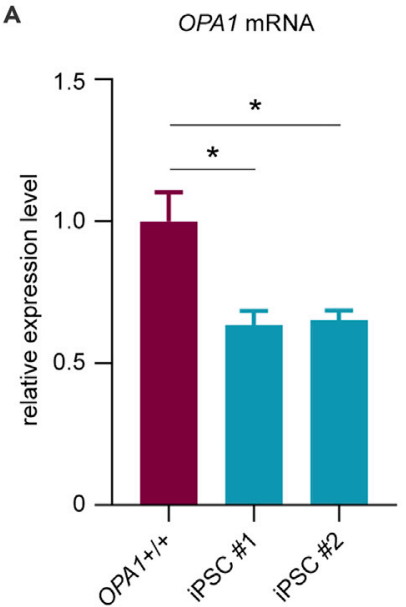

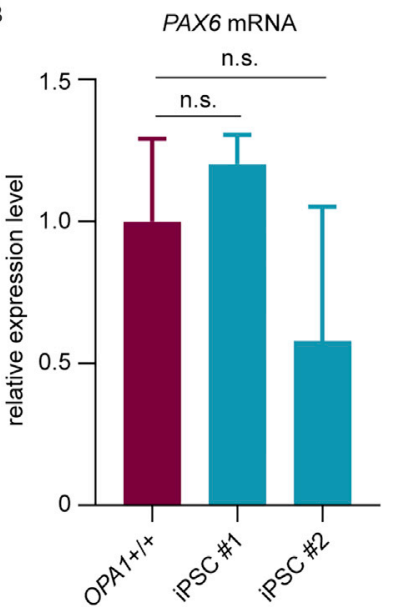

C

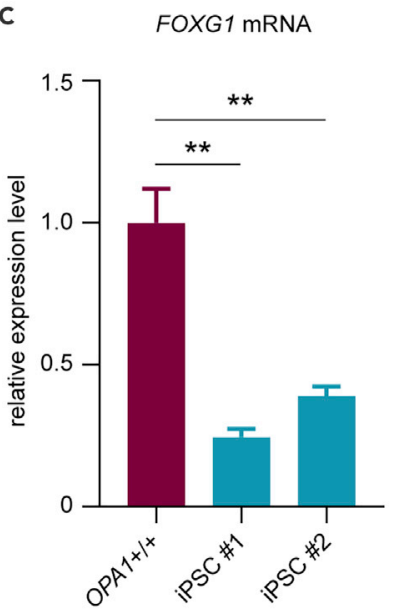

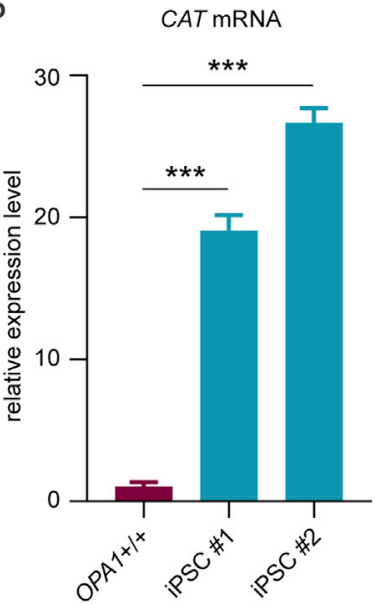

E
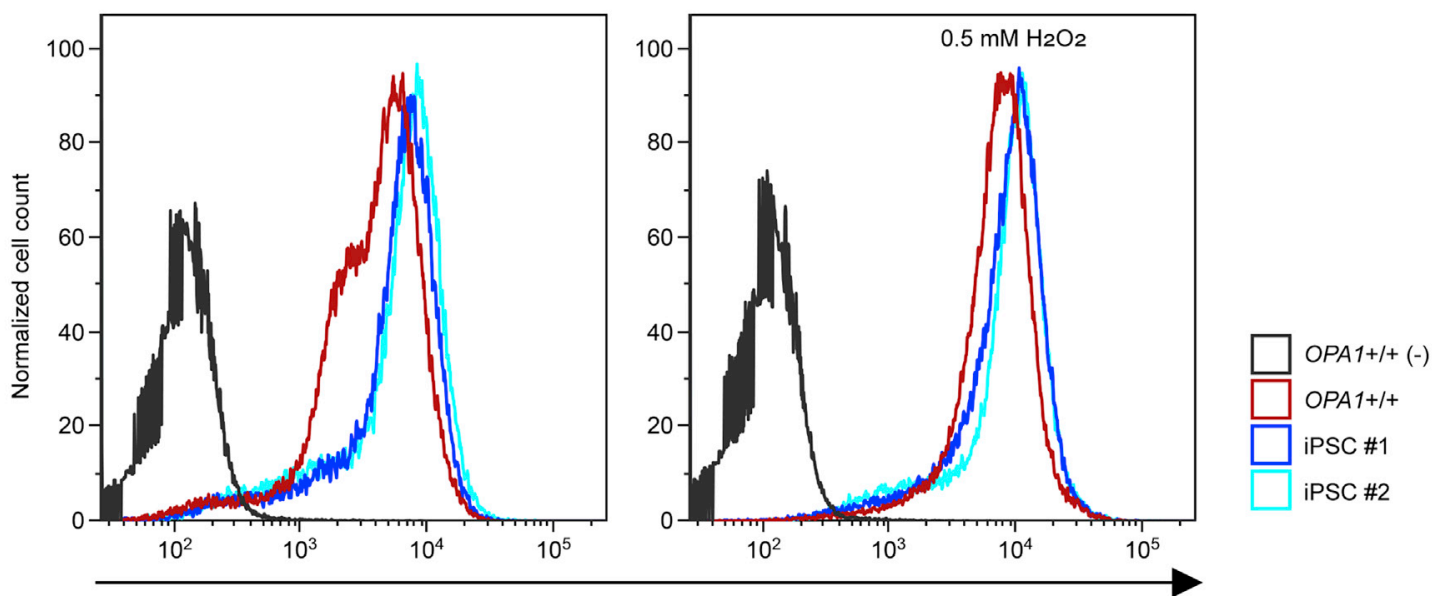

$\mathbf{F}$

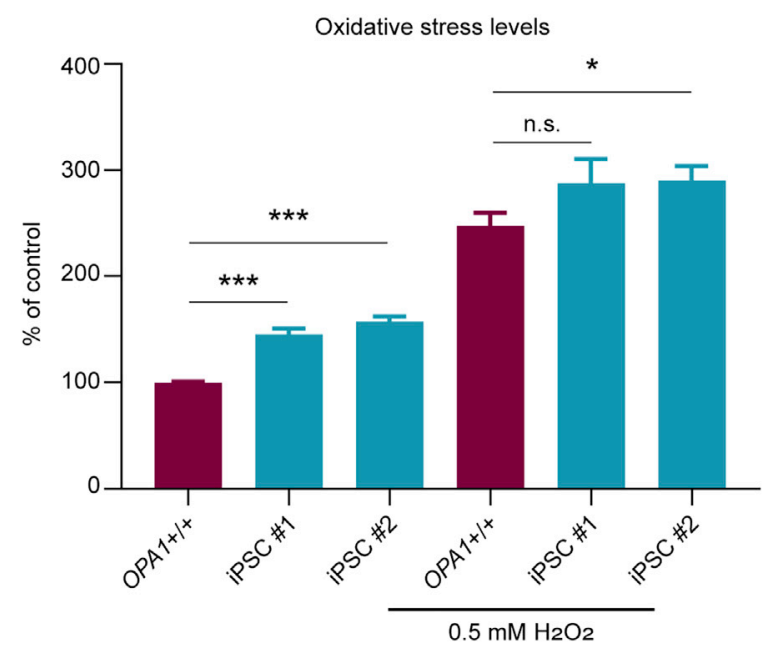

Figure 7. Patient-Derived iPSCs Harboring a Pathogenic OPA1 Mutation Exhibit Increased ROS Levels and Downregulation of FOXG1 (A-D) (A) OPA1 mRNA, (B) PAX6, (C) FOXG1, and (D) CAT mRNA expression levels in day 11 NPCs differentiated from iPSC lines derived from two donors diagnosed with ADOA

(E) Representative flow cytometry analysis of OPA1+/+ hESCs, iPSC \#1, and iPSC \#2 labeled with the oxidative stress indicator CM-H2DCFDA. Unlabeled hESCs (-) were used as negative control. hESCs and iPSCs that were incubated with $0.5 \mathrm{mM} \mathrm{H}_{2} \mathrm{O}_{2}$ were used as positive control. 
Figure 7. Continued

(F) Quantification of flow cytometry measurements in iPSC \#1 and iPSC \#2. Arithmetic mean values were normalized to the values of labeled OPA1+/+hESCs. Mean $\pm \mathrm{SEM}, \mathrm{N}=3$ independent experiments and $\mathrm{N}=2$ technical replicates. Student's test was used to analyze difference between two groups. $\mathrm{n}$.s. not significant. ${ }^{\star} p<0.05,{ }^{* \star} p<0.01,{ }^{* * *} p<0.001$

off-target effects by CRISPR-Cas9, we generated induced pluripotent stem cells (iPSCs) derived from two male donors who carry the inactivating OPA1 c.2873_2876delTTAG mutation. First, we assessed OPA1 mRNA expression and found that mRNA expression levels were reduced by $35 \%-40 \%$ compared with OPA1+/+ NPCs (Figure 7A). Next, we found that PAX6 mRNA expression levels were similar between patient iPSC- and hESC-derived NPCs (Figure 7B). Moreover, FOXG1 (Figure 7C) and CAT (Figure 7D) mRNA expression levels were down- and upregulated in patient iPSC-derived NPCs, respectively. Next, we used the ROS indicator CM-H2DCFDA and further demonstrated that ROS levels were markedly increased in patient-derived iPSCs (Figures 7E and 7F). Taken together, these results indicate that OPA1 haploinsufficiency is accompanied by aberrant nuclear DNA methylation and changed transcriptional circuitry.

\section{DISCUSSION}

Our study shows for the first time that OPA1 haploinsufficiency correlates with aberrant nuclear DNA methylation patterns as well as a significantly altered transcriptional circuitry and differentiation potential of NPCs (Figure 8).

The role of OPA1 during physiologic and pathologic neural development remains incompletely understood. We observed a severe loss of DLX1/DLX2-positive neurons when OPA1 protein levels were reduced by $50 \%$. Studies using mouse models have previously shown that DIx1 and DIx2 are expressed in the developing retinal neuroepithelium (Eisenstat et al., 1999) and are needed for the terminal differentiation of lateborn RGCs (de Melo et al., 2005). Moreover, D/x1/D/x2 double-null mice exhibit up to $30 \%$ reduced numbers of RGCs, whereas formation of other retinal neuronal cell types is not affected. In addition to Dlx1/2, loss of Sfrp1 expression (Esteve et al., 2003) and forced expression of NeuroD factors (Cherry et al., 2011) are known to dysregulate retinal cell diversity in mice and chicken. Strikingly, we find impaired expression of DLX1/2 and SFRP1 as well as an upregulation of NEUROD4 when OPA1 levels are reduced, which may explain the loss of RGCs observed in patients with ADOA.

Our transcriptome analysis in NPCs substantiates the relevance of balanced OPA1 levels for proper neural cell homeostasis. One TF that we find consistently repressed in our study is the pioneer factor FOXG1, which is predominantly expressed in the developing neuroepithelium, retina, and optic chiasm. A previous study found that FOXG1 is crucial for GABAergic neuronal formation and retinal development because Foxg1-/- mice exhibit eye abnormalities (Xuan et al., 1995). Importantly, Foxg1-/- telencephalic progenitors fail to generate Dlx1-and Dlx2-positive neurons (Xuan et al., 1995). Other evidence that links FOXG1 to the generation of $D L X 1 / 2+$ neurons stems from a recent report demonstrating that FOXG1 upregulation is responsible for the overproduction of GABAergic neurons in brain organoids derived from iPSCs obtained from patients with autism spectrum disorder (Mariani et al., 2015). In addition, studies in mice and chicken showed that loss of Foxg1 expression results in RGC axonal misprojection and pathfinding defects (Yuasa et al., 1996; Pratt et al., 2004). Our gene expression analyses in hESC-derived NPCs and neurons also suggest that FOXG1 is a direct regulator of human GABAergic neuronal formation and retinal development.

Recently, iPSCs derived from patients carrying heterozygous mutations in OPA1 and having an inherited form of Parkinson disease (PD) were generated and used to investigate dopaminergic neuronal development. The authors found fragmented mitochondria, a decrease in respiration, and a decrease in neuronal viability, as well as an increase in ROS (lannielli et al., 2018). Although we also observe an increase in ROS and sensitivity to apoptotic stimuli, we only find subtle changes in mitochondrial morphology including increased intercristae distance, reduced circumference, and a trend to more fragmented mitochondria in OPA $1+/-$ hESCs. Overall, the phenotype in PD patient-derived iPSCs seems more severe than the one observed in OPA1 haploinsufficient hESCs and ADOA patient-derived iPSCs. It is possible that this is because PD is a more complex disease with potential additional epigenetic and genetic changes in contrast to familial optic atrophy with a single-gene mutation. Other studies also show significant mitochondrial fragmentation in mouse cerebellar granule (Jahani-Asl et al., 2011) and HeLa cells (Arnoult et al., 2005). In these studies OPA1 expression was almost completely abolished, whereas in our cells $50 \%$ OPA 1 proteins remain, which might explain the mild changes in mitochondrial morphology in our 


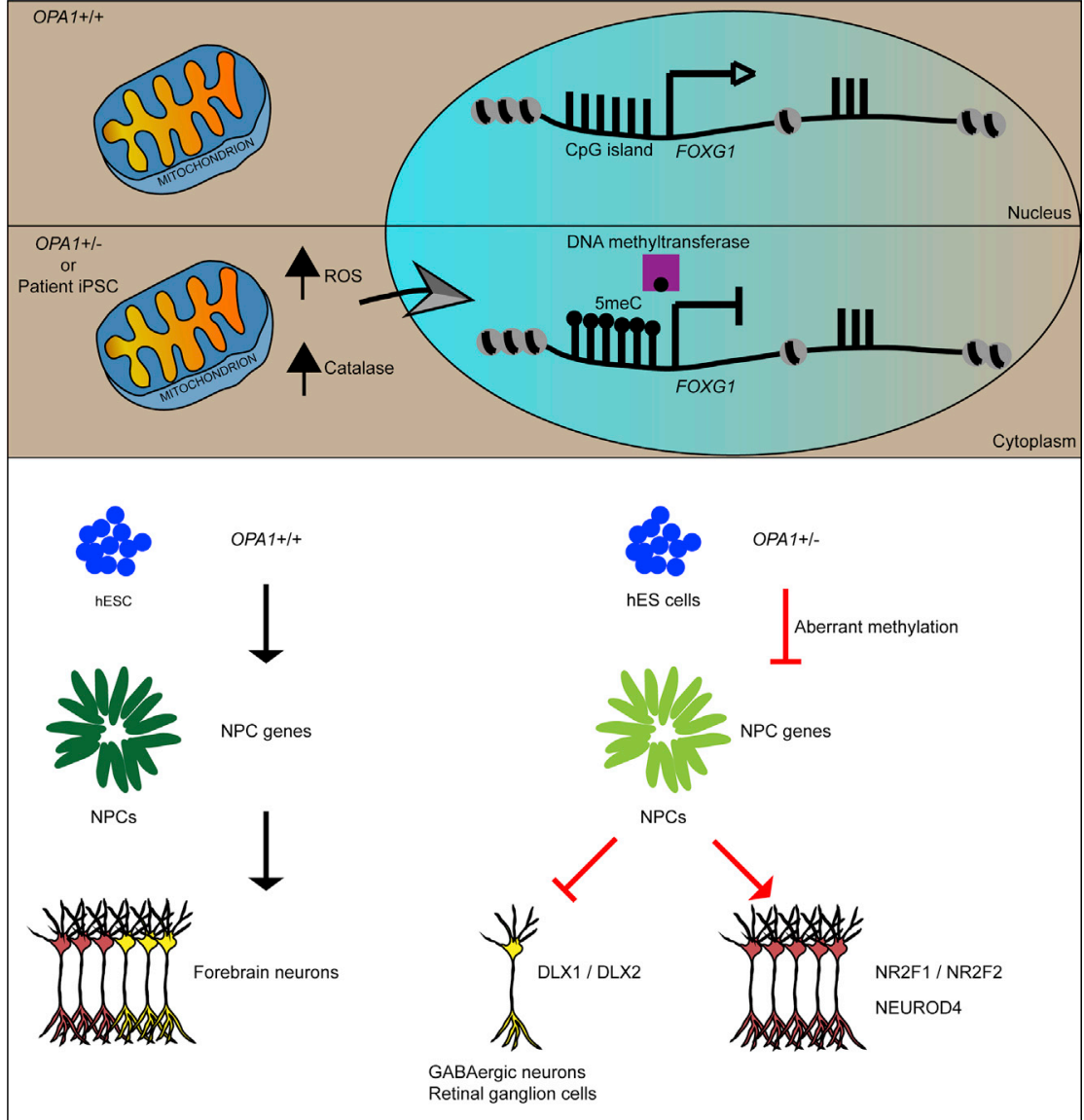

Figure 8. Schematics Illustrating the Metabolic and Epigenetic Changes in OPA1 Haploinsufficient Cells, Which May Contribute to Altered Neuronal Differentiation

cells. In line with this, a recent study using OPA1 haploinsufficient iPSCs derived from patients with syndromic parkinsonism also shows only subtle changes in the mitochondrial ultrastructure in iPSC-derived dopaminergic neurons (Jonikas et al., 2018).

A fascinating question is how OPA1 haploinsufficiency impacts on nuclear DNA methylation at specific sites, such as the FOXG1 and ATP10D promoters. Epigenetic pathways and transcriptional regulation are influenced by non-protein-coding RNA molecules. For instance, the murine long non-coding RNA Kcnq1ot1 recruits the DNA methyltransferase Dnmt1 to CpG islands of imprinted genes (Mohammad et al., 2010), and the natural antisense transcript AS1DHRS4 controls localization of DNMTs to the promoter regions of transcripts DHRS4L1 and DHRS4L2 (Li et al., 2012). Given that the DNA methylation changes we observe in OPA1+/- NPCs are loci specific, it is possible that expression and availability of non-coding RNAs that regulate DNA methylation is affected in OPA1+/- NPCs.

Enzymes catalyzing epigenetic changes require specific metabolites as sources of acetyl or methyl groups (Katada et al., 2012; Lu and Thompson, 2012). Small metabolites such as SAM, SAH, and alpha-ketoglutarate establish a link between mitochondrial metabolism and epigenetics (Matilainen et al., 2017). We find similar SAM/SAH and succinate/alpha-ketoglutarate levels in OPA1+/- and OPA1+/+ cells, suggesting that the observed changes in DNA methylation are not caused by alterations of these small metabolites. Previously, it has been shown that oxidative stress causes aberrant DNA methylation and transcriptional silencing (O'Hagan et al., 2011). The authors reveal that DNMT1 is targeted to damaged chromatin, forming a complex with DNMT3B and polycomb repressive complex 4 ( $\mathrm{O}^{\prime}$ Hagan et al., 2011), and show that hydrogen peroxide treatment causes a relocalization of these proteins to promoter CpG islands. Importantly, we find that OPA1 haploinsufficiency results in increased oxidative stress and ROS accumulation 
in OPA1+/- hESCs and ADOA patient-derived iPSCs. It is well possible that altered ROS levels play a role in the DNA methylation changes we described. Supporting this notion, mitochondrial dysfunction and increased ROS levels have recently been described to cause specific promoter hypermethylation and decreased expression of microRNA-663 in cancer cell lines (Carden et al., 2017). Exogenous and endogenous ROS are also known to affect neural stem cell renewal and proliferation. For instance, Le Belle et al. show that brain-derived neural progenitors maintain a high ROS status and are responsive to ROS stimulation leading to posttranslational oxidative inactivation of PTEN, and activation of PI3K/Akt signaling pathway, which is required for NPC self-renewal (Le Belle et al., 2011). Another study by Chui et al. demonstrated that selective removal of histone-lysine N-methyltransferase PRDM16 in NPCs leads to abnormal neuronal composition and organization in the mouse brain by inducing excessive ROS (Chui et al., 2020). Our results together with these studies suggest that activation of specific pathways by ROS signaling is context dependent and may influence various pathways during cell fate specification.

In conclusion, we provide evidence that OPA1 haploinsufficient cells exhibit increased oxidative stress, aberrant nuclear DNA methylation, and a significantly altered transcriptional circuitry.

\section{Limitations of the Study}

Although we addressed DNA methylation changes in the promoter regions of selected down-regulated genes, we did not explore this for up-regulated genes or in a global context. Moreover, we did not assess cell death under stress conditions in iPSC-derived neurons, and performed the mitochondrial respiration assay in two not three independent experiments, although each with 10-40 replicates. In addition, although we observed an increase in ROS that might contribute to the aberrant DNA methylation, we were not able to identify the exact mechanism by which OPA1 haploinsufficiency contributes to these changes in nuclear DNA methylation. Defining primary versus secondary changes related to OPA1 will be exciting to explore in the future.

\section{Resource Availability}

Lead Contact

Further information and requests for resources and reagents should be directed to and will be fulfilled by the Lead Contact, Judith Staerk (judith.staerk@ncmm.uio.no).

Materials Availability

There may be restrictions or delays to the availability of hESC and iPSC lines due to our need to maintain the stock and patient consent. Availability of iPSC lines will require a Materials Transfer Agreement.

Data and Code Availability

Access to data that support the findings of this study are available from the authors on reasonable request. RNA-seq information including all raw data has been deposited at Gene Expression Omnibus under GSE117976: https://www.ncbi.nlm.nih.gov/geo/query/acc.cgi?acc=GSE117976.

\section{METHODS}

All methods can be found in the accompanying Transparent Methods supplemental file.

\section{SUPPLEMENTAL INFORMATION}

Supplemental Information can be found online at https://doi.org/10.1016/j.isci.2020.101154.

\section{ACKNOWLEDGMENTS}

This work was supported by the Research Council of Norway, The Regional Health Authority for SouthEastern Norway, Novo Nordisk Foundation, Denmark, the University of Oslo, Norway and the European Union Seventh Framework Programme (FP7-PEOPLE-2013-COFUND) under grant agreement $n^{\circ} 609020$ - Scientia Fellows. We thank the patients for agreeing to participate in this study. We thank Marianne Smestad and Ulrikke Brinch Dahl at the core facility for Advanced EM at OUS, which is funded by the South East Regional Health Authority, Norway, for technical assistance. The HPLC-MS/MS analyses of DNA modifications were performed by the Proteomics and Modomics Experimental Core Facility (PROMEC), Norwegian University of Science and Technology (NTNU), Trondheim, Norway. PROMEC is funded by the Faculty of 
Medicine and Health Sciences at NTNU and the Central Norway Regional Health Authority, Norway. We thank the Medical Bioinformatics Centre of Turku Centre for Biotechnology for the copy number data analysis. The Centre is supported by the University of Turku, Åbo Akademi University, and Biocenter Finland, Finland.

\section{AUTHOR CONTRIBUTIONS}

S.C. and J.S. designed the study. J.S. generated genetically modified hESC and patient iPSC lines. S.C., A.C.-P., V.J., C.P., S.B., B.T., D.T.C., M.R., A. Brech, and S.H.B. performed experiments, A.H. performed bioinformatics analysis. A. Boschi provided primary patient material. S.C., A.H., V.J., A. Brech, S.H.B., E.A.N., L.H., K.T., and J.S. interpreted results. S.C. and J.S. wrote the manuscript. All authors commented and approved the final version.

\section{DECLARATION OF INTERESTS}

The authors declare no competing interests.

Received: October 29, 2019

Revised: April 3, 2020

Accepted: May 6, 2020

Published: June 26, 2020

\section{REFERENCES}

Akepati, V.R., Muller, E.C., Otto, A., Strauss, H.M., Portwich, M., and Alexander, C. (2008). Characterization of OPA1 isoforms isolated from mouse tissues. J. Neurochem. 106, 372-383.

Alavi, M.V., Bette, S., Schimpf, S., Schuettauf, F., Schraermeyer, U., Wehrl, H.F., Ruttiger, L., Beck S.C., Tonagel, F., Pichler, B.J., et al. (2007). A splice site mutation in the murine Opal gene features pathology of autosomal dominant optic atrophy. Brain 130, 1029-1042.

Alexander, C., Votruba, M., Pesch, U.E., Thiselton, D.L., Mayer, S., Moore, A., Rodriguez, M., Kellner, U., Leo-Kottler, B., Auburger, G., et al. (2000). OPA 1 , encoding a dynamin-related GTPase, is mutated in autosomal dominant optic atrophy linked to chromosome 3q28. Nat. Genet. 26, 211-215.

Anderson, S.A., Eisenstat, D.D., Shi, L., and Rubenstein, J.L. (1997). Interneuron migration from basal forebrain to neocortex: dependence on Dlx genes. Science 278, 474-476.

Arnoult, D., Grodet, A., Lee, Y.J., Estaquier, J., and Blackstone, C. (2005). Release of OPA1 during apoptosis participates in the rapid and complete release of cytochrome $\mathrm{c}$ and subsequent mitochondrial fragmentation. J. Biol. Chem. 280, 35742-35750.

Beaubien, F., and Cloutier, J.-F. (2009).

Differential expression of slitrk family members in the mouse nervous system. Dev. Dyn. 238, 32853296.

Carden, T., Singh, B., Mooga, V., Bajpai, P., and Singh, K.K. (2017). Epigenetic modification of miR-663 controls mitochondria-to-nucleus retrograde signaling and tumor progression. J. Biol. Chem. 292, 20694-20706.

Chambers, S.M., Fasano, C.A., Papapetrou, E.P., Tomishima, M., Sadelain, M., and Studer, L. (2009). Highly efficient neural conversion of human ES and iPS cells by dual inhibition of SMAD signaling. Nat. Biotechnol. 27, 275-280.

Chan, D.C. (2012). Fusion and fission: interlinked processes critical for mitochondrial health. Annu. Rev. Genet. 46, 265-287.

Cherry, T.J., Wang, S., Bormuth, I. Schwab, M., Olson, J., and Cepko, C.L. (2011). NeuroD factors regulate cell fate and neurite stratification in the developing retina. J. Neurosci. 31, 7365-7379.

Chui, A, Zhang, O., Dai, O, and Shi, S.H. (2020). Oxidative stress regulates progenitor behavior and cortical neurogenesis. Development 147, dev184150.

Davies, V.J., Hollins, A.J., Piechota, M.J., Yip, W. Davies, J.R., White, K.E., Nicols, P.P., Boulton, M.E., and Votruba, M. (2007). Opa1 deficiency in a mouse model of autosomal dominant optic atrophy impairs mitochondrial morphology, optic nerve structure and visual function. Hum. Mol. Genet. 16, 1307-1318.

de Melo, J., Du, G., Fonseca, M., Gillespie, L.-A. Turk, W.J., Rubenstein, J.L.R., and Eisenstat, D.D. (2005). Dlx1 and Dlx2 function is necessary for terminal differentiation and survival of late-born retinal ganglion cells in the developing mouse retina. Development 132, 311-322.

Deglincerti, A., Etoc, F., Ozair, M.Z., and Brivanlou, A.H. (2016). Chapter six - selforganization of spatial patterning in human embryonic stem cells. In Current Topics in Developmental Biology, 116, P.M. Wassarman ed (Academic Press), pp. 99-113.

Del Dotto, V., Mishra, P., Vidoni, S., Fogazza, M. Maresca, A., Caporali, L., McCaffery, J.M. Cappelletti, M., Baruffini, E., Lenaers, G., et al. (2017). OPA1 isoforms in the hierarchical organization of mitochondrial functions. Cell Rep. $19,2557-2571$.
Delettre, C. Griffoin, J.M., Kaplan, J., Dollfus، H., Lorenz, B., Faivre, L., Lenaers, G., Belenguer, P., and Hamel, C.P. (2001). Mutation spectrum and splicing variants in the OPA1 gene. Hum. Genet. 109, 584-591.

Delettre, C., Lenaers, G., Griffoin, J.M., Gigarel, N., Lorenzo, C., Belenguer, P., Pelloquin, L., Grosgeorge, J., Turc-Carel, C., Perret, E., et al. (2000). Nuclear gene OPA1, encoding a mitochondrial dynamin-related protein, is mutated in dominant optic atrophy. Nat. Genet. 26, 207-210

Eisenstat, D.D., Liu, J.K., Mione, M., Zhong, W. Yu, G., Anderson, S.A., Ghattas, I., Puelles, L., and Rubenstein, J.L.R. (1999). DLX-1, DLX-2, and DLX5 expression define distinct stages of basal forebrain differentiation. J. Comp. Neurol. 414, 217-237.

Esteve, P., Trousse, F., Rodríguez, J., and Bovolenta, P. (2003). SFRP1 modulates retina cell differentiation through a $\beta$-catenin-independent mechanism. J. Cell Sci. 116, 2471-2481.

Herrera, F.J., Yamaguchi, T., Roelink, H., and Tjian, R. (2014). Core promoter factor TAF9B regulates neuronal gene expression. Elife 3 , e02559.

Hevner, R.F., Shi, L., Justice, N., Hsueh, Y., Sheng, M., Smiga, S., Bulfone, A., Goffinet, A.M., Campagnoni, A.T., and Rubenstein, J.L. (2001). Tbr1 regulates differentiation of the preplate and layer 6. Neuron 29, 353-366.

Ho, T.T., Warr, M.R., Adelman, E.R., Lansinger, O.M., Flach, J., Verovskaya, E.V., Figueroa, M.E. and Passegue, E. (2017). Autophagy maintains the metabolism and function of young and old stem cells. Nature 543, 205-210.

lannielli, A., Bido, S., Folladori, L., Segnali, A. Cancellieri, C., Maresca, A., Massimino, L., Rubio, A., Morabito, G., Caporali, L., et al. (2018)

Pharmacological inhibition of necroptosis 
protects from dopaminergic neuronal cell death in Parkinson's disease models. Cell Rep. 22, 20662079

Jahani-Asl, A , Pilon-Larose, K, Xu, W., Maclaurin J.G., Park, D.S., Mcbride, H.M., and Slack, R.S. (2011). The mitochondrial inner membrane GTPase, optic atrophy 1 (Opa1), restores mitochondrial morphology and promotes neuronal survival following excitotoxicity. J. Biol. Chem. 286, 4772-4782

Jonikas, M., Madill, M., Mathy, A., Zekoll, T., Zois, C.E., Wigfield, S., Kurzawa-Akanbi, M., Browne, C. Sims, D. Chinnery, P.F., et al. (2018). Stem cell modeling of mitochondrial parkinsonism reveals key functions of OPA1. Ann. Neurol. 83, 915-925.

Katada, S., Imhof, A., and Sassone-Corsi, P. (2012). Connecting threads: epigenetics and metabolism. Cell 148, 24-28.

Le Belle, J.E., Orozco, N.M., Paucar, A.A., Saxe, J.P., Mottahedeh, J., Pyle, A.D., Wu, H., and Kornblum, H.I. (2011). Proliferative neural stem cells have high endogenous ROS levels that regulate self-renewal and neurogenesis in a PI3K/ Akt-dependant manner. Cell Stem Cell 8, 59-71.

Letinic, K., Zoncu, R., and Rakic, P. (2002). Origin of GABAergic neurons in the human neocortex. Nature 417, 645-649.

Li, Q., Su, Z., Xu, X., Liu, G., Song, X., Wang, R. Sui, X. Liu, T. Chang, $X$. and Huang, D. (2012). AS1DHRS4, a head-to-head natural antisense transcript, silences the DHRS4 gene cluster in cis and trans. Proc. Natl. Acad. Sci. U S A 109,14110 14115.
Lu, C., and Thompson, C.B. (2012). Metabolic regulation of epigenetics. Cell Metab. 16, 9-17.

MacVicar, T., and Langer, T. (2016). OPA1 processing in cell death and disease - the long and short of it. J. Cell Sci. 129, 2297-2306.

Mariani, J., Coppola, G., Zhang, P., Abyzov, A., Provini, L., Tomasini, L., Amenduni, M., Szekely, A., Palejev, D., Wilson, M., et al. (2015). FOXG1 Dependent dysregulation of GABA/glutamate neuron differentiation in autism spectrum disorders. Cell 162, 375-390.

Matilainen, O., Quiros, P.M., and Auwerx, J. (2017). Mitochondria and epigenetics - crosstalk in homeostasis and stress. Trends Cell Biol. 27 453-463.

Mirzayans, F., Walter, M.A., Berry, F.B., Link, B.A. Skarie, J.M., Hudson, T.J., Fortin, Y., and Raymond, V. (2007). FOXC1 is required for cell viability and resistance to oxidative stress in the eye through the transcriptional regulation of FOX01A. Hum. Mol. Genet. 17, 490-505.

Mohammad, F., Mondal, T., Guseva, N., Pandey G.K. and Kanduri, C. (2010). Kcnq1ot1 noncoding RNA mediates transcriptional gene silencing by interacting with Dnmt1. Development 137, 24932499

Nasca, A., Rizza, T., Doimo, M., Legati, A., Ciolfi, A. Diodato, D. Calderan, C. Carrara, G.. Lamantea, E., Aiello, C., et al. (2017). Not only dominant, not only optic atrophy: expanding the clinical spectrum associated with OPA1 mutations. Orphanet J. Rare Dis. 12, 89.
O'Hagan, Heather, M., Wang, W., Sen, S., Destefano Shields, C., Lee, S.S., Zhang, Y.W. Clements, E.G., Cai, Y., van Neste, L., et al. (2011) Oxidative damage targets complexes containing DNA methyltransferases, SIRT1, and polycomb members to promoter $\mathrm{CpG}$ islands. Cancer Cell 20, 606-619.

Pratt, T., Tian, N.M.M.-L., Simpson, T.I., Mason, J.O., and Price, D.J. (2004). The winged helix transcription factor Foxg1 facilitates retinal ganglion cell axon crossing of the ventral midline in the mouse. Development 131, 37733784.

Shi, Y., Kirwan, P., and Livesey, F.J. (2012). Directed differentiation of human pluripotent stem cells to cerebral cortex neurons and neural networks. Nat. Protoc. 7, 1836-1846.

Spiegel, R., Saada, A., Flannery, P.J., Burte, F., Soiferman, D., Khayat, M., Eisner, V., Vladovski, E., Taylor, R.W., Bindoff, L.A., et al. (2016). Fatal infantile mitochondrial encephalomyopathy, hypertrophic cardiomyopathy and optic atrophy associated with a homozygous OPA1 mutation. J. Med. Genet. 53, 127-131.

Xuan, S.H., Baptista, C.A., Balas, G., Tao, W.F., Soares, V.C., and Lai, E. (1995). Winged helix transcription factor $\mathrm{Bf}-1$ is essential for the development of the cerebral hemispheres. Neuron 14, 1141-1152.

Yuasa, J., Hirano, S., Yamagata, M., and Noda, M. (1996). Visual projection map specified by topographic expression of transcription factors in the retina. Nature 382, 632-635. 
iScience, Volume 23

Supplemental Information

Optic Atrophy 1 Controls Human Neuronal

Development by Preventing Aberrant

Nuclear DNA Methylation

Safak Caglayan, Adnan Hashim, Artur Cieslar-Pobuda, Vidar Jensen, Sidney Behringer, Burcu Talug, Dinh Toi Chu, Christian Pecquet, Marie Rogne, Andreas Brech, Sverre Henning Brorson, Erlend Arnulf Nagelhus, Luciana Hannibal, Antonella Boschi, Kjetil Taskén, and Judith Staerk 


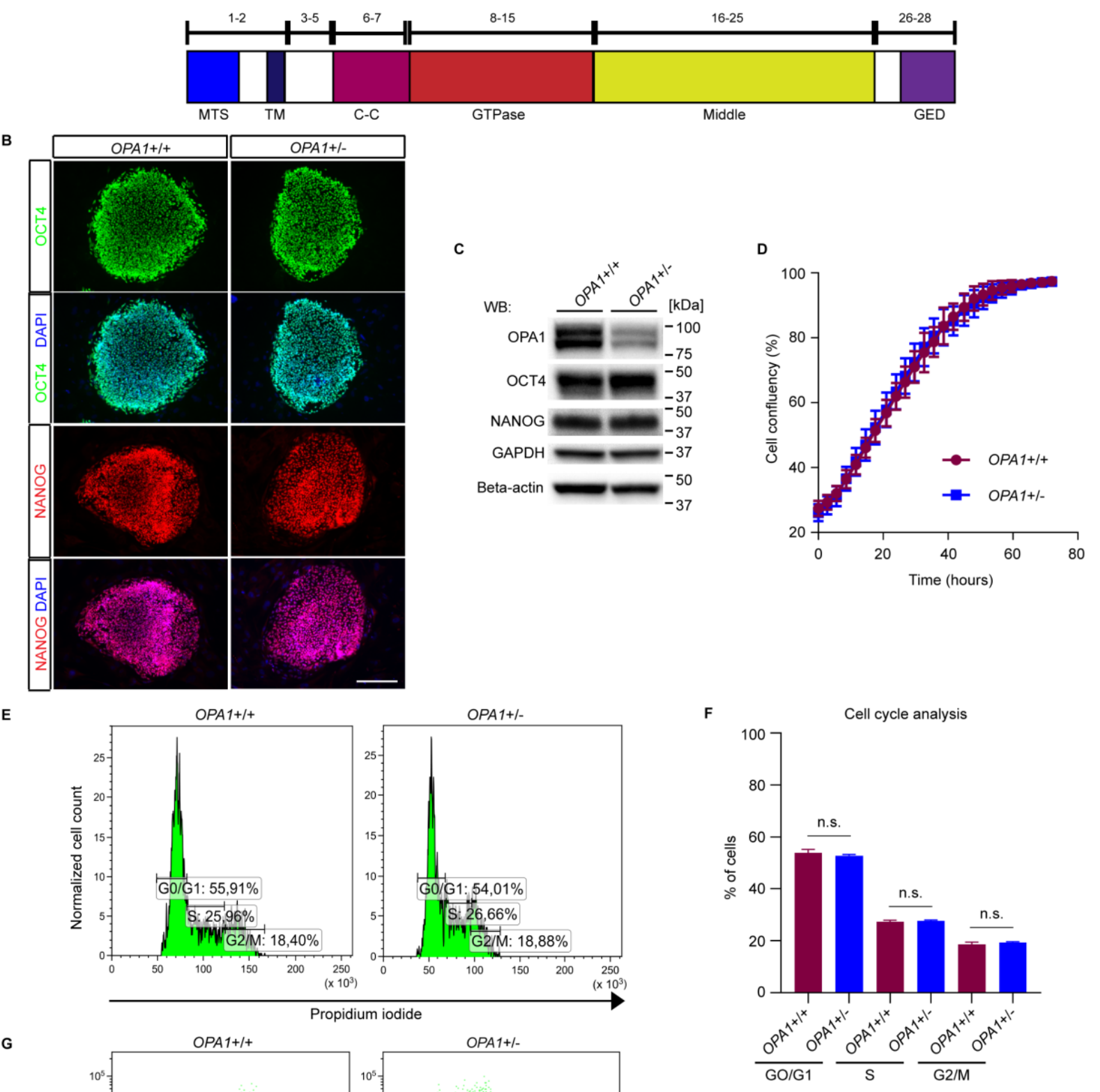


Figure S1. Schematic representation of OPA1 and characterization of OPA1+/- hESCs. Related to Figure 1. A) Genomic and protein domain organization of OPA1. Exon 4b and 5b are alternatively spliced exons in OPA1 isoform 1. Numbers above protein domains denote exons coding for the respective domains. MTS: mitochondria transport sequence; TM: transmembrane domain; C-C: coiled-coiled domain; GED: GTPase effector domain. Protein domains identified based on (Delettre et al., 2000) and RNA isoforms are depicted according to (Delettre et al., 2001, Akepati et al., 2008). B) Immunostainings of OPA1+/+ and OPA1+/- hESCs using OCT4 and NANOG antibodies. DAPI was used to stain nuclei. Scale bar $200 \mu \mathrm{m}$. C) Representative immunoblotting images showing OPA1, OCT4, and NANOG protein levels. GAPDH and betaactin were used as loading controls. D) Proliferation rate of hESCs quantified using the Incucyte live cell imaging instrument. E) Representative flow cytometry analysis of $O P A 1+/+$ and $O P A 1+/-$ hESCs labeled with fluorescent DNA stain propidium iodide (PI). F) Quantification of different cell cycle stages using PI labeling in OPA1+/+ and OPA1+/- hESCs. Mean $\pm \mathrm{SEM}, \mathrm{N}=4$ independent experiments and $\mathrm{N}=2$ technical replicates. G) Representative FACS analysis of $O P A 1+/+$ and OPA1+/- hESCs labeled with proliferation indicator Ki67 antibody and PI. Similar results were obtained in $\mathrm{N} \geq 3$ independent experiments. Student's $t$ test was used to analyze difference between two groups. n.s. not significant. 
A

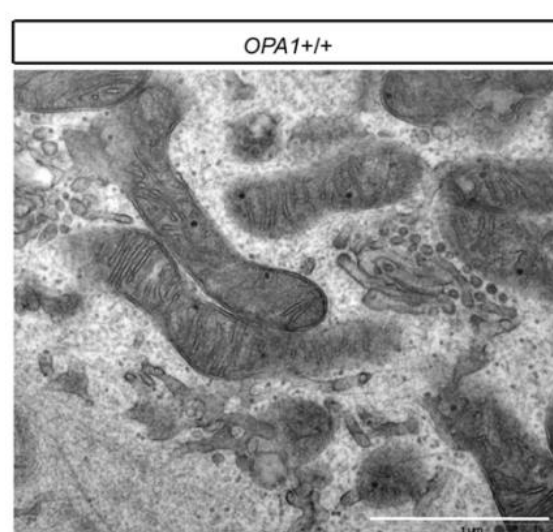

B

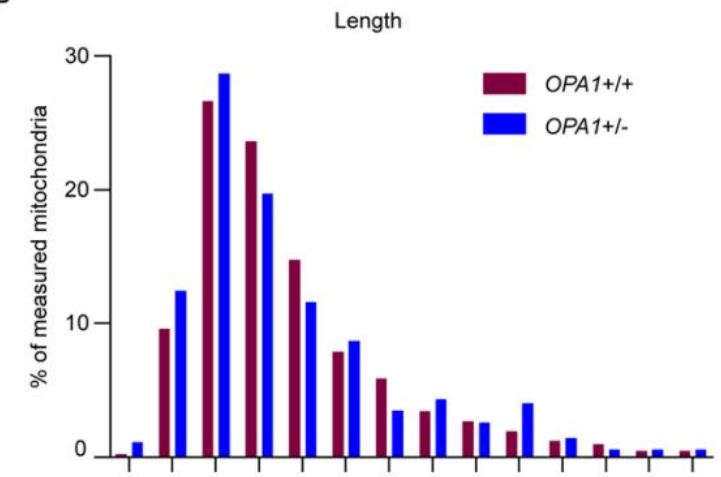

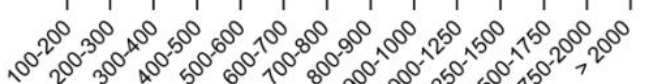

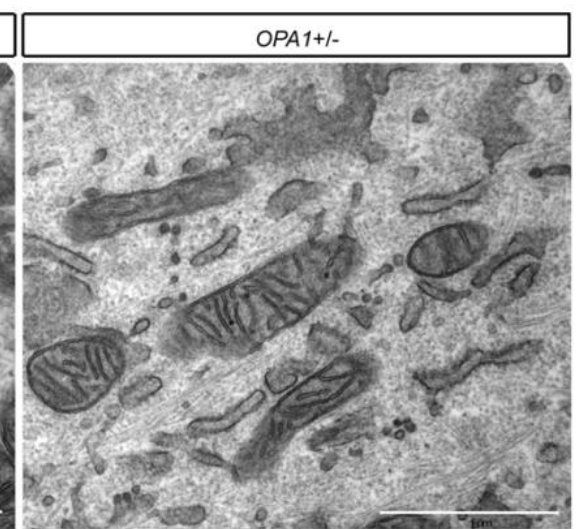

D

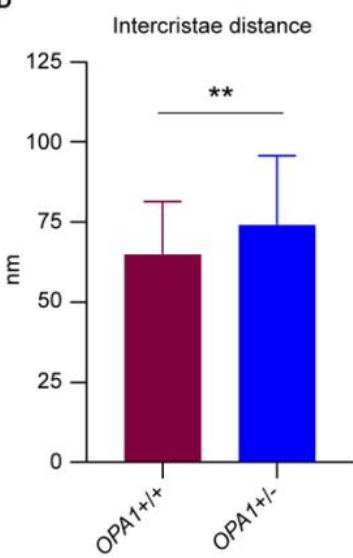

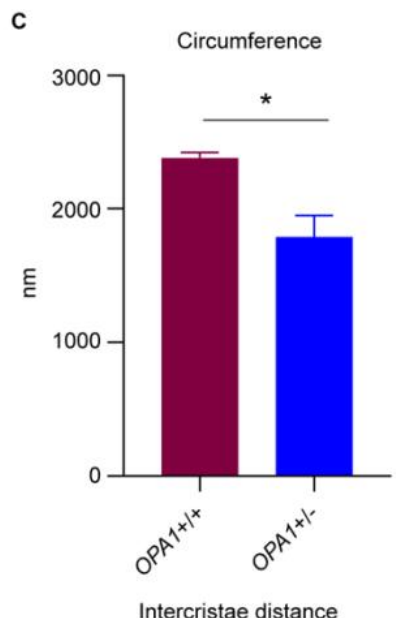

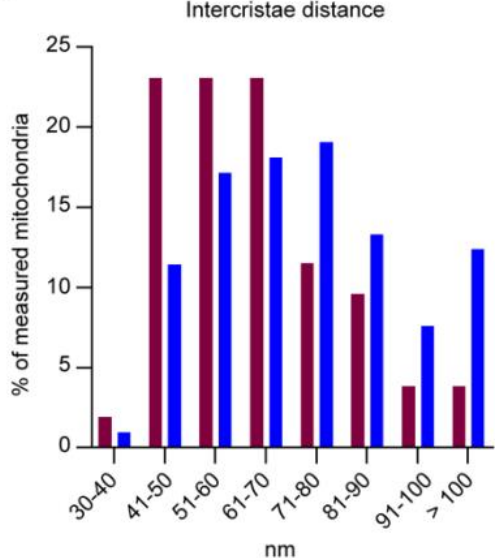

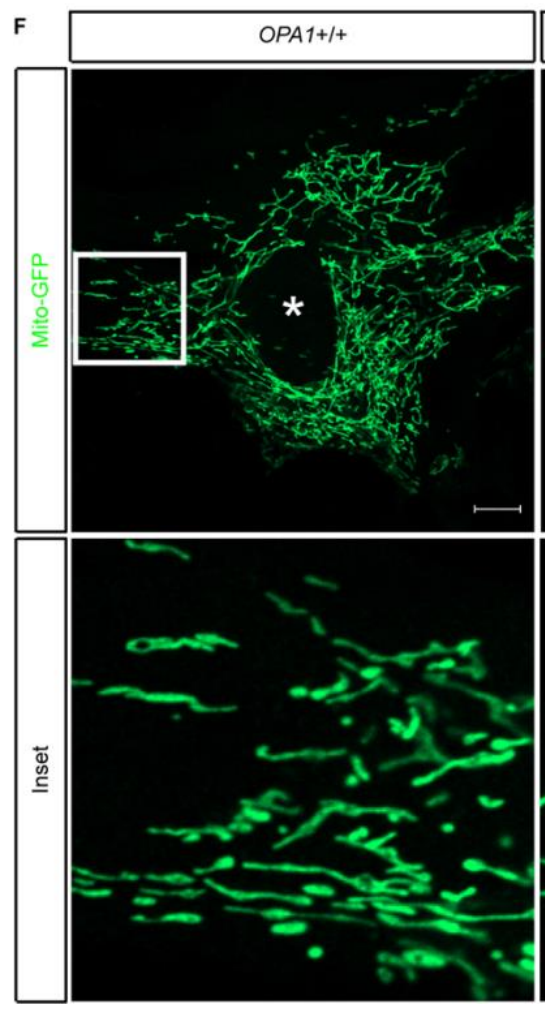

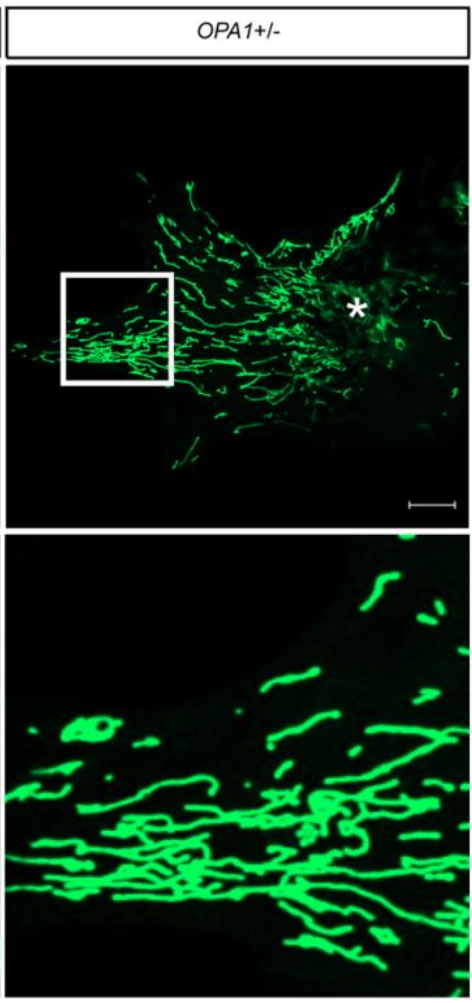

G

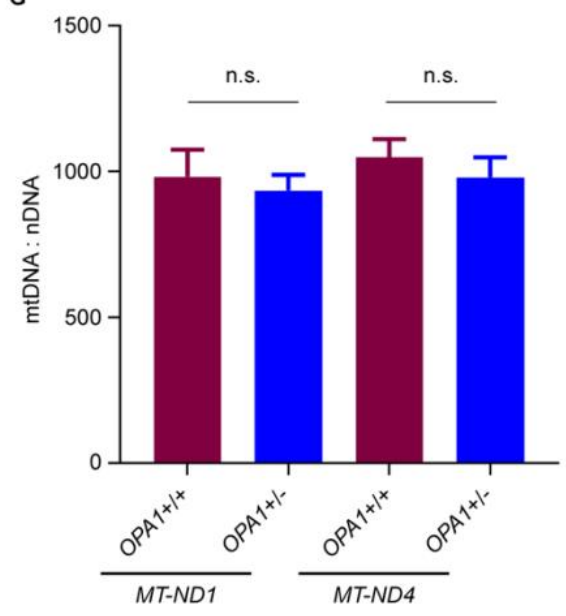


Figure S2. Subtle changes in mitochondrial morphology in OPA1+/- hESCs. Related to Figure 1. A) Representative transmission electron microscopy images of $O P A 1+/+$ and $O P A 1+/-$ hESCs. Scale bar $1 \mu \mathrm{m}$. B) Circumference was reduced in OPA1+/- compared to OPA1+/+ hESCs. C) Mitochondria measurements were binned into categories of length intervals. D) Intercristae distances were significantly increased in OPA1+/- compared to OPA1+/+ hESCs. E) Binning of intercristae distances into categories of distance intervals. F) Confocal microscopy images of mito-GFP transduced hESCs. * denotes nucleus. Scale bar $10 \mu \mathrm{m}$. G) Quantification of mitochondrial DNA content relative to nuclear DNA amount by qPCR. Mean $\pm S E M, N \geq 3$ independent experiments. Student's test was used to analyze difference between two groups. n.s. not significant, ${ }^{*}=p<0.05,{ }^{* *}=p<0.01$. 

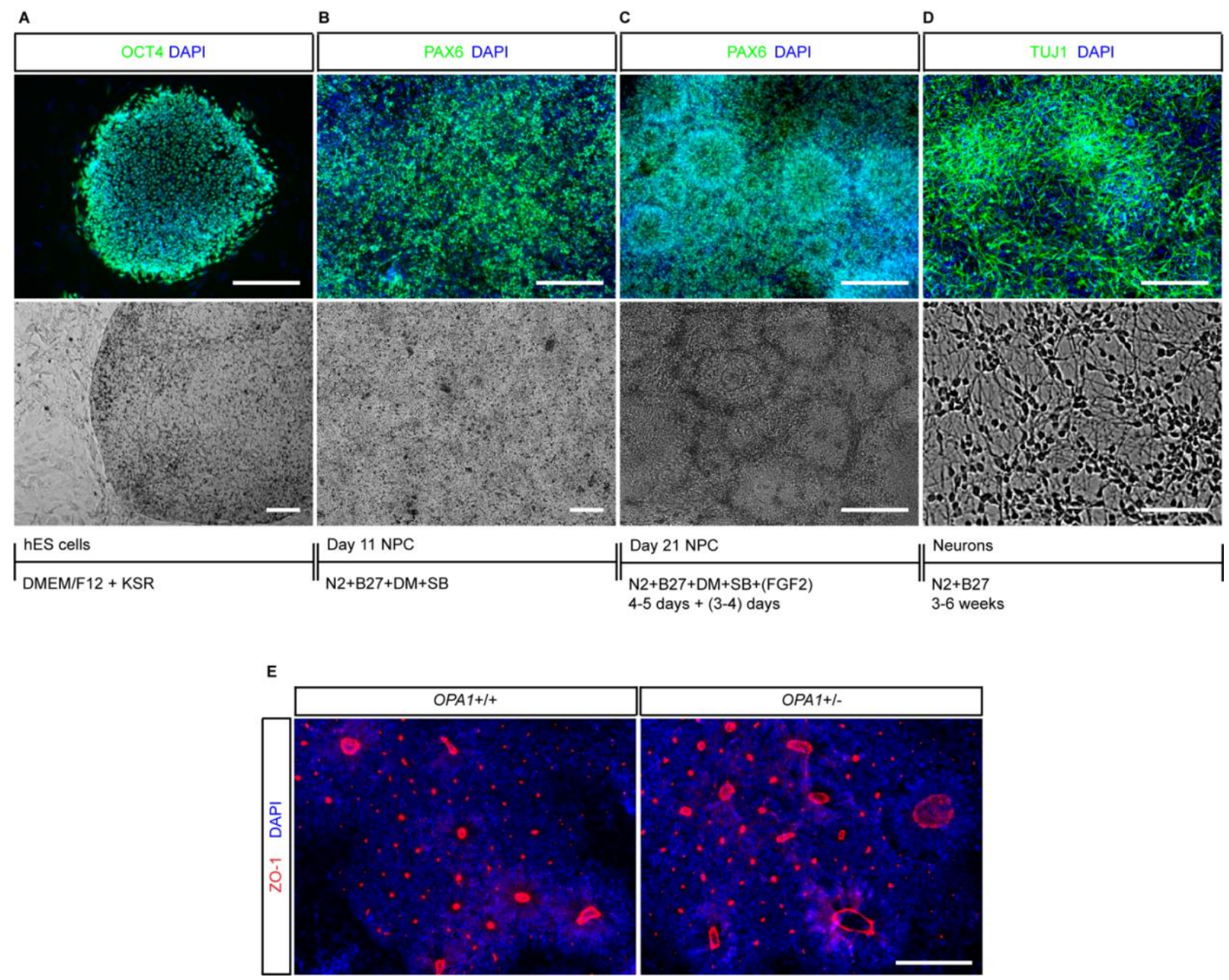

Figure S3. NPC derivation and neuronal differentiation. Related to Figures 2, 3, 4, and 5. A) OCT4 positive hESCs were maintained in DMEM/F12 media containing FGF2. B) Neural differentiation was induced with media containing N2/B27 together with dual SMAD inhibitors. Bright-field and immunostaining images of day 11 NPCs. C) PAX6-positive NPCs forming rosettes as shown by bright-field and fluorescence microscopy in week 3 NPCs. D) Neuronal layers as shown by bright-field and immunostaining images were derived 3-6 weeks after NPC passaging. E) Rosette formation by NPCs shown by ZO-1 marker. DAPI was used to stain nuclei. Scale bar $50 \mu \mathrm{m}$ in panel $\mathrm{D}$ and $200 \mu \mathrm{m}$ in panels $\mathrm{A}, \mathrm{B}, \mathrm{C}$ and $\mathrm{E}$. 
A

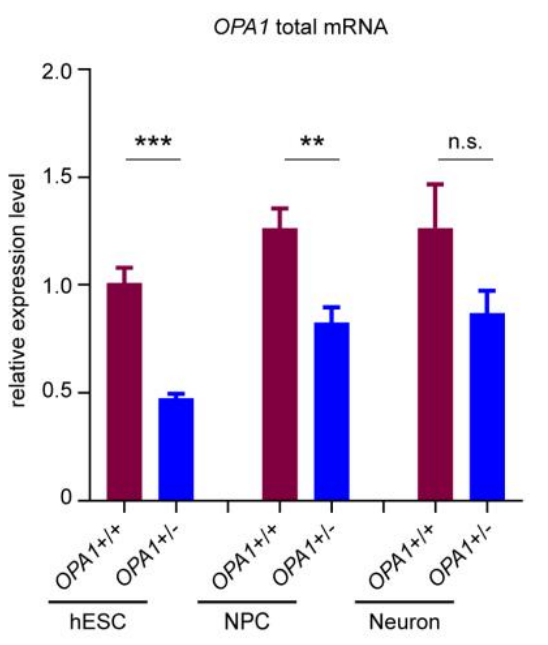

B

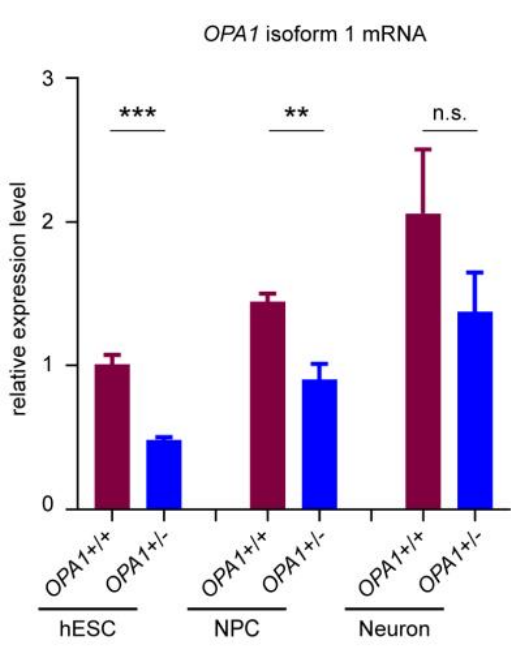

C

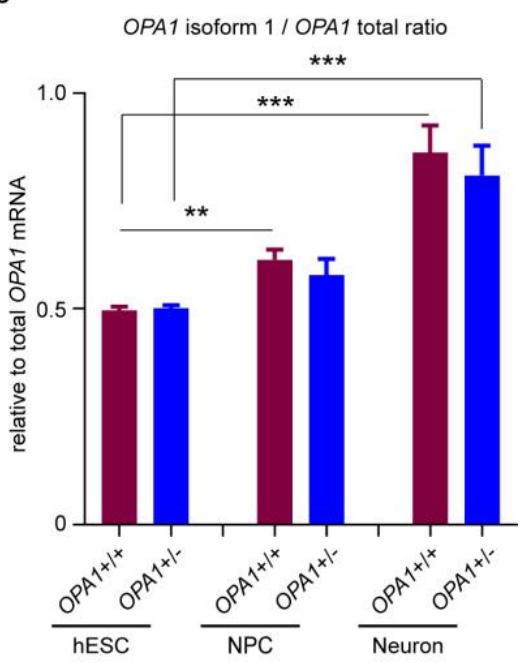

D
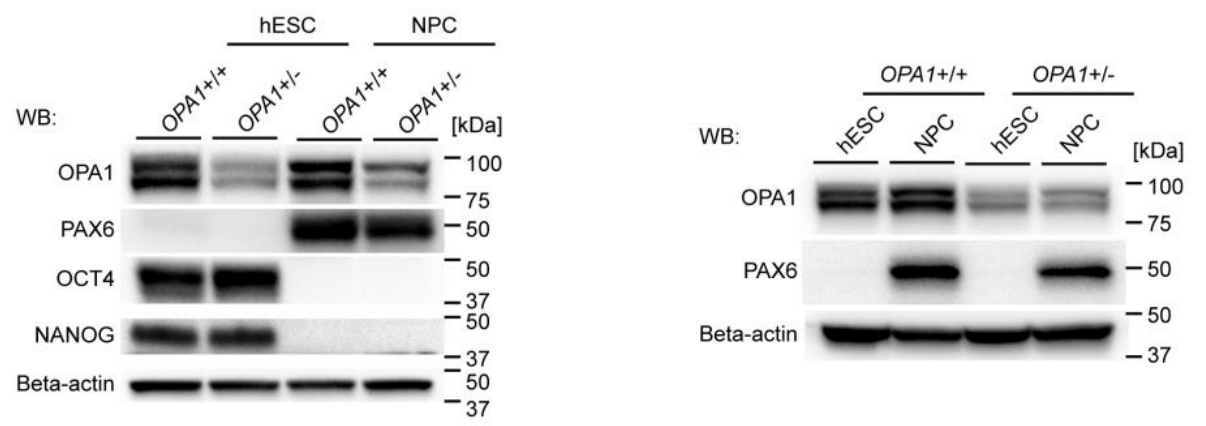

$E$

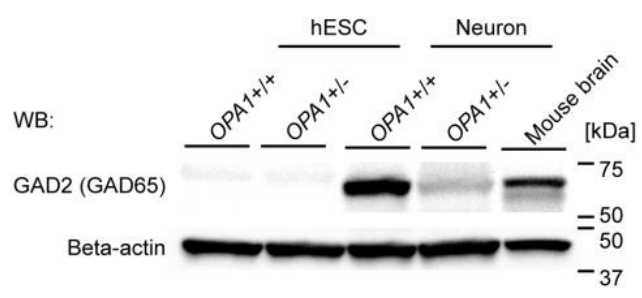

Figure S4. Characterization of OPA1+/- and OPA1+/+ hESCs, NPCs and neurons. Related to Figures 1, 2, 3, and 4. A) QRT-PCR analysis of OPA1 mRNA expression levels in hESCs, week 3 NPCs and neurons. B) OPA1 isoform 1 transcript levels quantified by qRT-PCR. C) Ratio of isoform 1 to total OPA1 transcript levels. Mean \pm SEM, $N \geq 3$ independent experiments. D) Immunoblotting validating NPC derivation and OPA1 upregulation in NPCs derived from hESCs. OCT4 and NANOG were used as pluripotency marker, PAX6 was used as neural differentiation marker. Beta-actin was used as loading control. E) Immunoblotting image of GAD2/GAD65 in $O P A 1+/+$ and OPA1+/- hESCs and neurons. Beta actin was used as loading control. Student's t test was used to analyze difference between two groups. n.s. not significant. ${ }^{* *}=p<0.01,{ }^{* * *}=$ $\mathrm{p}<0.001$. 
A

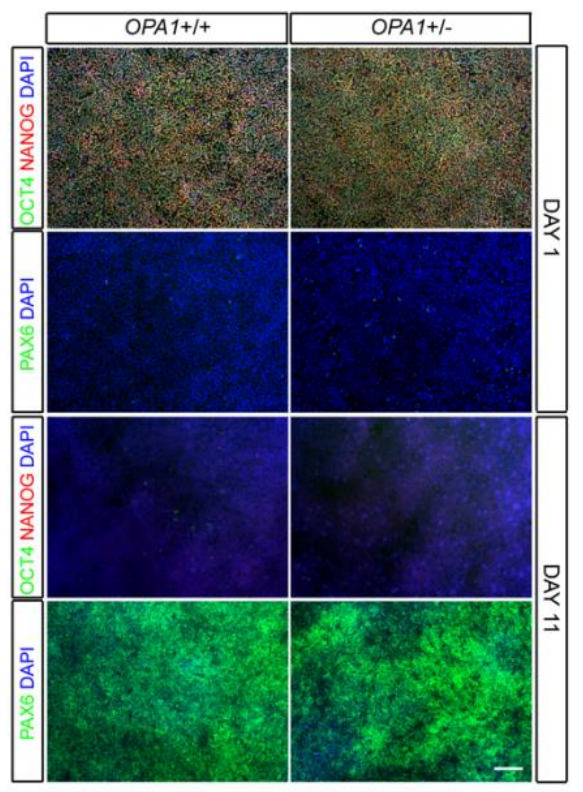

C

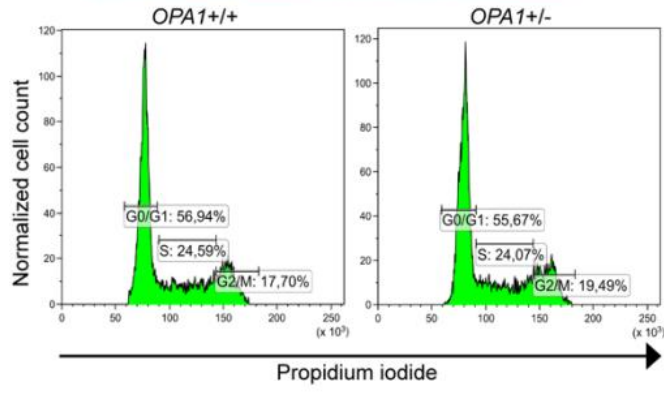

E

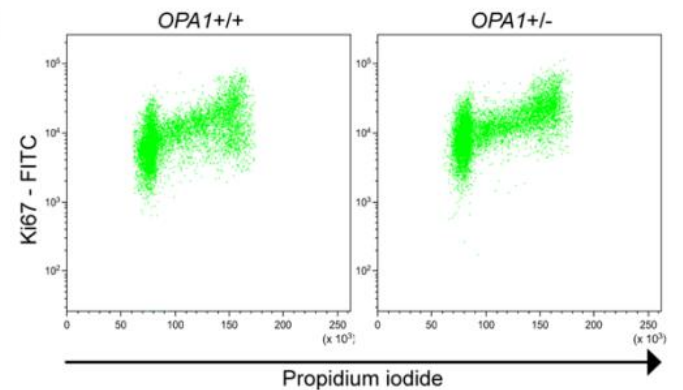

G

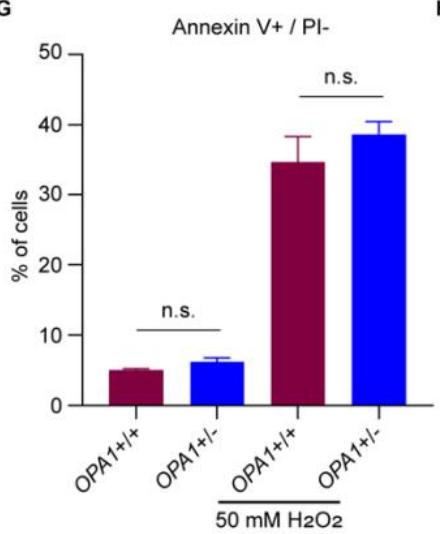

B
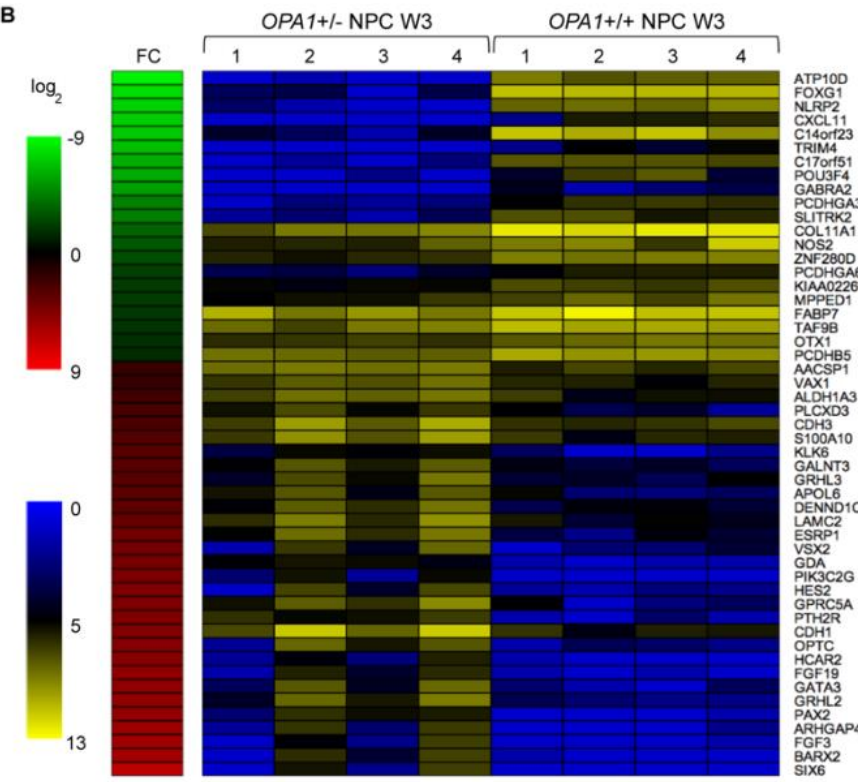

D
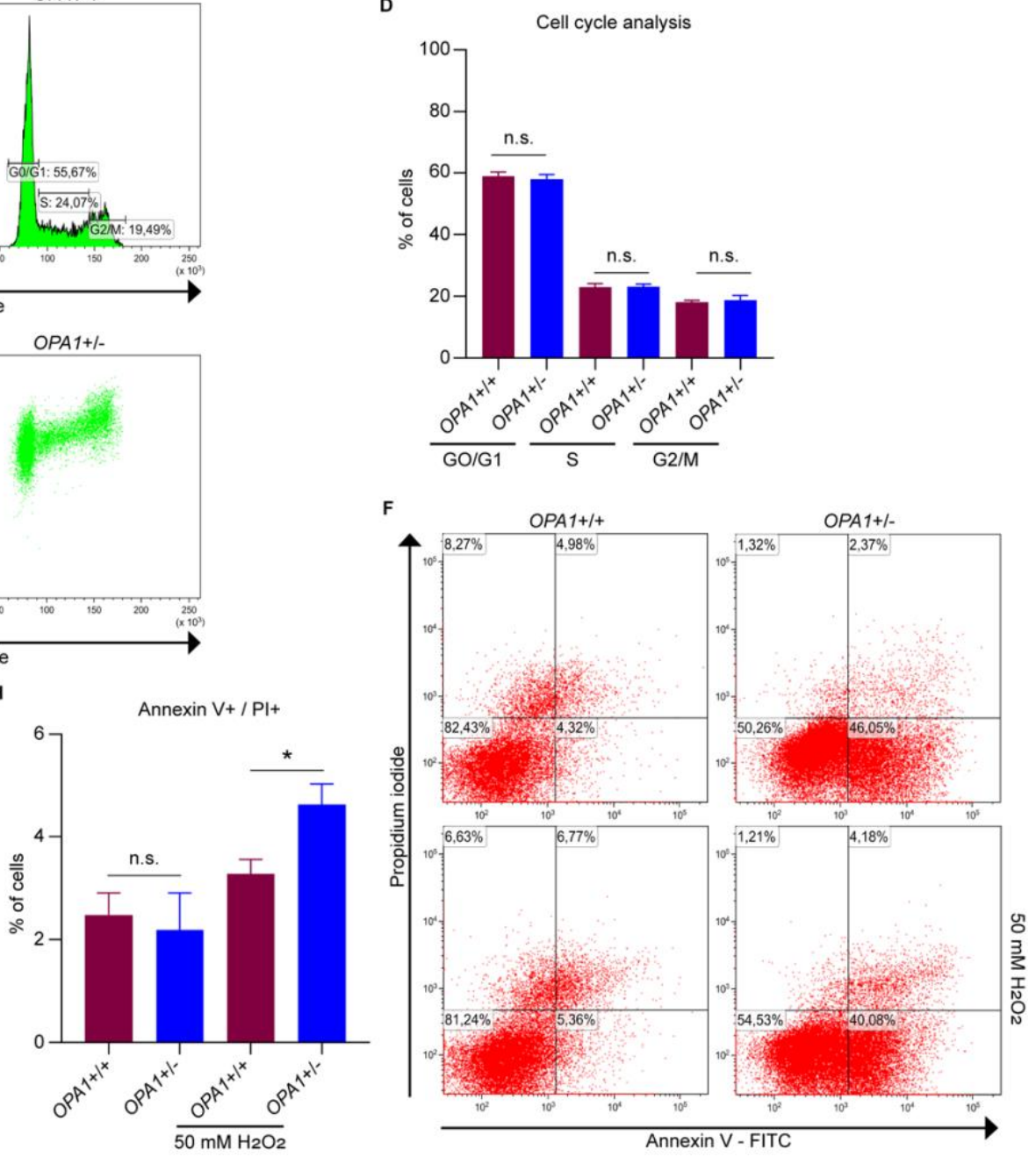
Figure S5. Transcriptional analysis of OPA1+/+ and OPA1+/- week 3 NPCs. Related to Figure 5 and Table S3. A) Immunostaining of hESC layers on day 1 before induction of neural differentiation and of NPC layers at day 11 of differentiation. OCT4 and NANOG were used as pluripotency marker, PAX6 was used as neural differentiation marker. Scale bar $200 \mu \mathrm{m}$. B) Log2 fold-change and expression heat-maps of selected down- (green) and up- (red) regulated genes in OPA1+/- compared to OPA1+/+ week 3 NPCs. FC = fold change. Expression intensities are displayed from blue (low expression) to yellow (high expression). C) Representative flow cytometry analysis of $O P A 1+/+$ and OPA1+/- NPCs labeled with fluorescent DNA stain propidium iodide (PI). D) Quantification of cell cycle stages using PI labeling in OPA1+/+ and OPA1+/-NPCs. Mean \pm SEM, $N=3$ independent experiments and $\mathrm{N}=2$ technical replicates. E) Representative FACS analysis of OPA1+/+ and OPA1+/- NPCs labeled with the proliferation indicator Ki67 antibody and $\mathrm{Pl}$. Similar results were obtained in $\mathrm{N}=3$ independent experiments. F) Representative flow cytometry analysis of OPA1+/+ and OPA1+/- NPCs labeled with the earlyapoptosis indicator Annexin V and late-apoptosis indicator PI. NPCs were incubated with $50 \mathrm{mM}$ $\mathrm{H}_{2} \mathrm{O}_{2}$ to assess sensitivity to apoptotic stimuli. G) Quantification of early-apoptotic Annexin $V_{+} /$ PI- NPCs. Percentage of labeled cells in total gated cells are shown. H) Quantification of lateapoptotic Annexin $\mathrm{V}_{+} / \mathrm{Pl}+\mathrm{NPCs}$. Percentage of labeled cells in total gated cells are shown. Mean \pm SEM, $N \geq 4$ independent experiments and $N=2$ technical replicates. Student's $t$ test was used to analyze difference between two groups. n.s. not significant, ${ }^{*}=p<0.05$. 
A

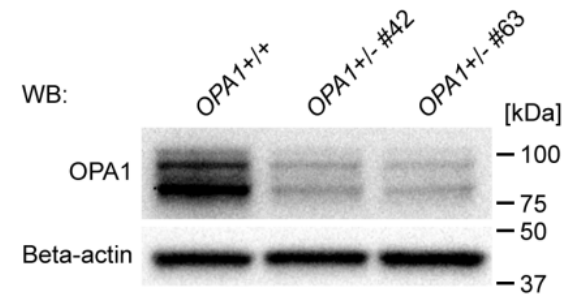

B
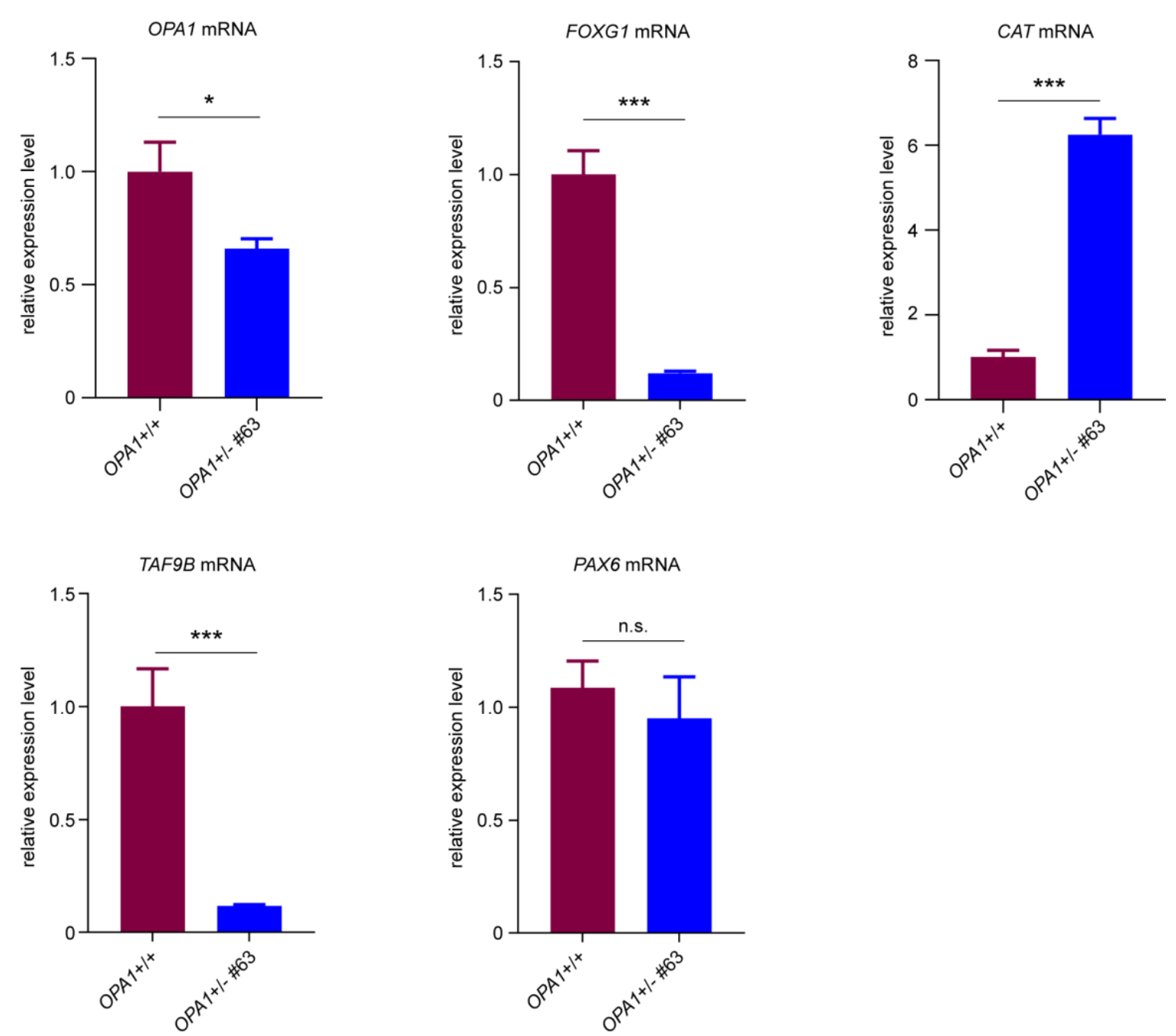

Figure S6. Confirmation of key findings in the second, independently derived OPA1+/hESC line \#63. Related to Figures 5 and 6. A) Immunoblotting of OPA1 in OPA1+/+ and OPA1+/- hESC lines \#42 and \#63. Beta-actin was used as loading control. B) OPA1, PAX6, FOXG1, TAF9B and CAT mRNA expression levels in NPCs derived from OPA1+/+ and OPA1+/\#63 hESCs. mRNA levels were normalized to transcript levels in OPA1+/+ NPCs. Mean \pm SEM, $\mathrm{N} \geq 3$ independent samples were analyzed for each genotype. Student's $t$ test was used to analyze the differences between two groups. n.s. not significant, ${ }^{*}=p<0.05,{ }^{* * *}=p<0.001$. 
A

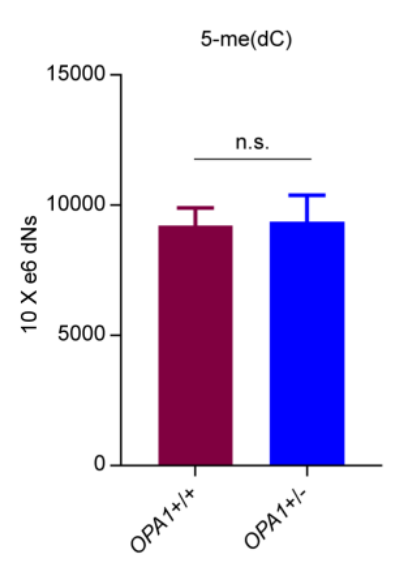

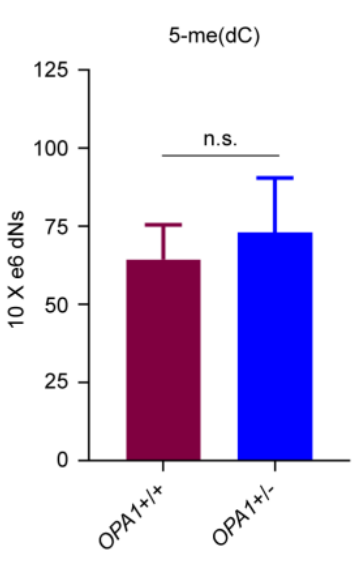

B

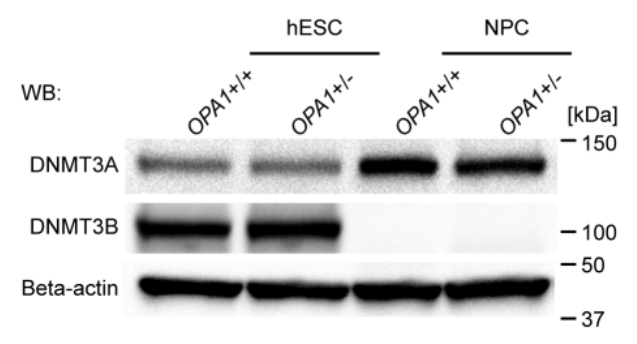

c

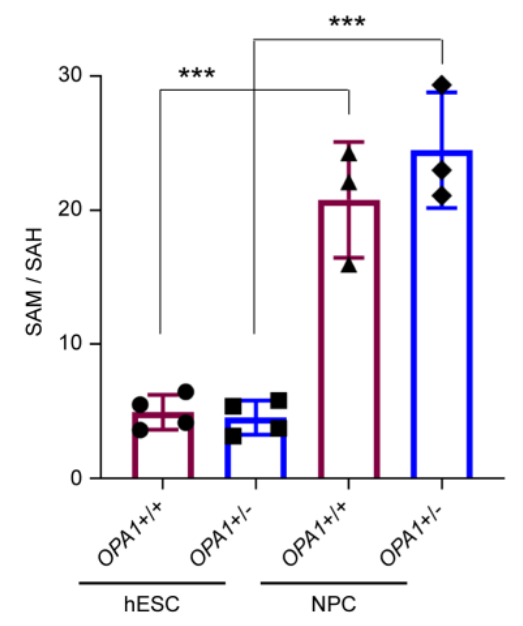

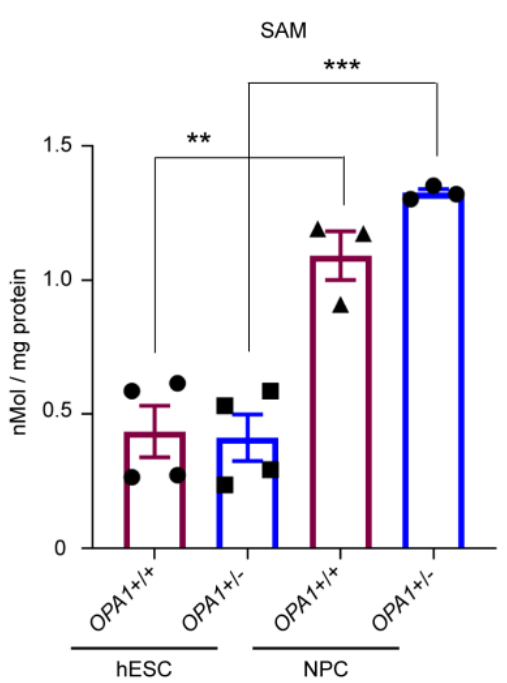

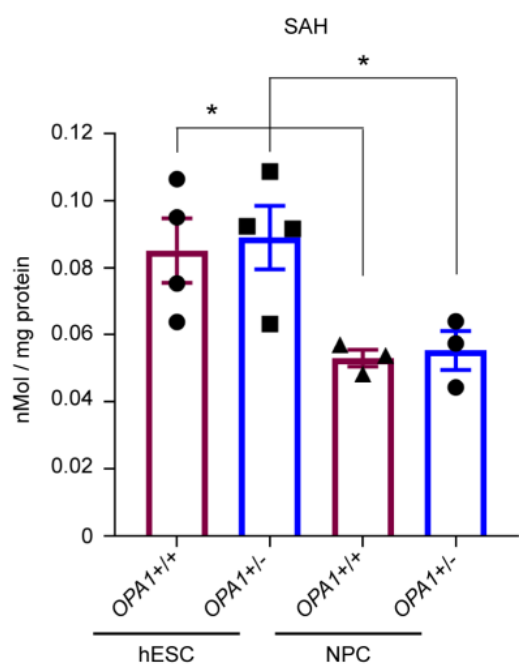

Figure S7. Mass spectrometry and immunoblotting analysis of epigenetic and metabolic changes. Related to Figure 6. A) Total methylation and hydroxymethylation levels were analyzed in OPA1+/+ and OPA1+/- week 3 NPCs. Quantification of 5-me $(\mathrm{dC})$ and 5-hm(dC) levels as determined by mass spectrometry. Mean \pm SEM, $N=3$ independent samples were analyzed for each genotype. B) Immunoblotting of DNMT3A and DNMT3B in OPA1+/+ and OPA1+/hESCs and NPCs. C) SAM / SAH ratio in OPA1+/+ and OPA1+/- hESCs and NPCs measured by mass spectrometry analysis. SAM and SAH levels were quantified in control and OPA1+/- hESCs and NPCs. Mean \pm SEM, $N \geq 3$ independent samples were assessed for each genotype and cell type. Student's t test was used to analyze the differences between two groups. n.s. not significant. ${ }^{*}=p<0.05,{ }^{* *}=p<0.01,{ }^{* * *}=p<0.001$. 


\section{Transparent Methods}

Table S4. Oligonucleotides used in this study. Related to Figures 1-7, and Figures S4 and S6.

\begin{tabular}{|c|c|}
\hline Oligonucleotide name & Sequence $\left(5^{\prime}\right.$-> $\left.3^{\prime}\right)$ \\
\hline ATP10D promoter bisulfite $\mathrm{FW}$ & TGAGATTGTAAGGGTTTTGGG \\
\hline ATP10D promoter bisulfite RV & TCACTTAAACATCTCCСAAACTC \\
\hline ATP10D RT-PCR FW & AAGTGGCAGACATTGGGATAG \\
\hline ATP10D RT-PCR RV & GAATCATGTTGGAAAGCCGTG \\
\hline B2M PCR FW & CACTGAAAAAGATGAGTATGCC \\
\hline B2M PCR RV & AACATTCCCTGACAATCCC \\
\hline CAT RT-PCR FW & CAGATAGCCTTCGACCCAAG \\
\hline CAT RT-PCR RV & GTAGGGACAGTTCACAGGTATATG \\
\hline$D L X 1 \mathrm{RT}-\mathrm{PCR} F \mathrm{~F}$ & CAGCCCCTACATCAGTTCG \\
\hline DLX1 RT-PCR RV & TCCCTTGCCATTGAAGCG \\
\hline$D L X 2$ RT-PCR FW & CTTACTCCGCCAAGAGCAG \\
\hline DLX2 RT-PCR RV & TCCCGTTCACTATCCGAATTTC \\
\hline FOXG1 RT-PCR FW & АССТАСТСССТСАAССССТ \\
\hline FOXG1 RT-PCR RV & AAAAGTAACTGGTCTGGCCC \\
\hline FOXG1 5'UTR bisulfite FW & TGGTTGTTGTTTTTGTTATATGATTT \\
\hline FOXG1 5'UTR bisulfite RV & ACTTAAAAATCATTTTCACCTCTTTC \\
\hline GABRA2 RT-PCR FW & CATTTGTGTTCTCTGCCCTAATTG \\
\hline GABRA2 RT-PCR RV & CTGTATCATAACGGAAGCCTTTTC \\
\hline GRHL2 RT-PCR FW & AAGTCCAGTTTCACCAGAGG \\
\hline GRHL2 RT-PCR RV & CTTCGGGTATTGAATGGAGG \\
\hline GRHL3 RT-PCR FW & GATATCCTGAAAACCTCCCCG \\
\hline GRHL3 RT-PCR RV & ACTCGCCTGACTTGATGTG \\
\hline MT-ND1 PCR FW & ACGCCATAAAACTCTTCACCAAAG \\
\hline MT-ND1 PCR RV & GGGTTCATAGTAGAAGAGCGATGG \\
\hline MT-ND4 PCR FW & ACCTTGGCTATCATCACCCGAT \\
\hline MT-ND4 PCR RV & AGTGCGATGAGTAGGGGAAGG \\
\hline OPA1 genotyping FW & GGCATCTCGAATCACCGTCTCTGACACC \\
\hline OPA1 genotyping RV & GGTGCCTGTCAGGCTCTTGCGG \\
\hline OPA1 gRNA1 top & CACCGCGACTACGTCGGGCCGCTG \\
\hline OPA1 gRNA1 bottom & AAACCAGCGGCCCGACGTAGTCGC \\
\hline OPA1 gRNA2 top & CACCGCTGGGCTTACGTTCGGAGC \\
\hline OPA1 gRNA2 bottom & AAACGCTCCGAACGTAAGCCCAGC \\
\hline OPA1 isoform $1 \mathrm{RT}$-PCR FW & AGACCTTGTAAAGTTAGCACCAG \\
\hline OPA1 isoform $1 \mathrm{RT}$-PCR RV & TGTCTGACACCTTTCTAAAATGC \\
\hline OPA1 total isoform RT-PCR FW & CCAGCAAGATTAGCTACGAGAC \\
\hline OPA1 total isoform RT-PCR RV & CACAATGTCAGGCACAATCC \\
\hline PAX6 RT-PCR FW & CTAAGGATGTTGAACGGGCA \\
\hline PAX6 RT-PCR RV & TTCTCCGTTGGAACTGATGG \\
\hline SIX6 RT-PCR FW & GACAAAGGGACCGAGCG \\
\hline SIX6 RT-PCR RV & CTTGCTGGATAGACTGGCG \\
\hline SLITRK2 RT-PCR FW & GGAGATGGGCAGGATTTAGT \\
\hline SLITRK2 RT-PCR RV & ACACTGAGGAACCAAACGC \\
\hline TAF9B RT-PCR FW & CGATTAAGTGTTGGTGCTGTTAG \\
\hline TAF9B RT-PCR RV & GTGGACTGAGAAGGTGGAATC \\
\hline
\end{tabular}




\begin{tabular}{|l|l|}
\hline TBR1 RT-PCR FW & AACAATGGGCAGATGGTGGTT \\
\hline TBR1 RT-PCR RV & GTTCACTTCCACCACATGCA \\
\hline THUMPD2 RT-PCR FW & GTCAGCGACTCACAGTTACTAG \\
\hline THUMPD2 RT-PCR RV & CACTTTCTGAAGGCAATGGC \\
\hline VAX1 RT-PCR FW & TCTATCGGCTGGAGATGGAG \\
\hline VAX1 RT-PCR RV & CCTGGTCCTTCTTCTGCTTG \\
\hline VSX2 RT-PCR FW & ACCAGACCAAGAAACGGAAG \\
\hline VSX2 RT-PCR RV & TTGAATGCCTTCTCCAGCTC \\
\hline ZNF280D RT-PCR FW & TGTATCCCAACTTCTGAGCAC \\
\hline ZNF280D RT-PCR RV & TCAGCCATGTTAGGTCTTGC \\
\hline $18 S R R N A$ RT-PCR FW & CTACCACATCCAAGGAAGGCA \\
\hline $18 S R R N A$ RT-PCR RV & TTTTTCGTCACTACCTCCCCG \\
\hline
\end{tabular}


Table S5. Antibodies used in this study. Related to Figures 1-6, Figure S1, and Figures S3-7.

\begin{tabular}{|l|l|l|}
\hline Antibody name (Clone) & Company & Catalog \# \\
\hline Mouse anti-beta Actin (C4) & Santa Cruz & SC-47778 \\
\hline Mouse anti-DLX2 (B-5) & Santa Cruz & SC-393879 \\
\hline Rabbit anti-DNMT3A & Cell Signaling Technologies & 2160 S \\
\hline Rabbit anti-DNMT3B & Sigma & HPA001595 \\
\hline Rabbit anti-DNM1L (D6C7) & Cell Signaling Technologies & 8570 S \\
\hline Rabbit anti-FOXG1 & Active Motif & 61211 \\
\hline Rabbit anti-GAD1 (1G10.2) & Millipore & MAB5406 \\
\hline Mouse anti-GAD2 (A-3) & Santa Cruz & SC-377145 \\
\hline Rabbit anti-NANOG (D73G4) & Cell Signaling Technologies & 4903 S \\
\hline Mouse anti-OCT3/4 (C-10) & Santa Cruz & SC-5279 \\
\hline Mouse anti-OPA1 (18/OPA1) & BD Biosciences & 612606 \\
\hline Rabbit anti-PAX6 (D3A9V) & Cell Signaling Technologies & $60433 S$ \\
\hline Sheep anti-PAX6 & R\&D Systems & AF8150 \\
\hline Rabbit anti-PKC (C24E6) & Cell Signaling Technologies & $9368 S$ \\
\hline Mouse anti-TBR1 (G-5) & Santa Cruz & SC-376258 \\
\hline Mouse anti-ZO1 (ZO1-1A12) & Thermo Fisher Scientific & $33-9100$ \\
\hline Alexa-488 Donkey anti-mouse IgG & Thermo Fisher Scientific & A21202 \\
\hline Alexa-488 Donkey anti-sheep IgG & Thermo Fisher Scientific & A11015 \\
\hline Alexa-488 Goat anti-rabbit IgG & Thermo Fisher Scientific & A11034 \\
\hline Alexa-488 Rabbit anti-Ki67 & Cell Signaling Technologies & $11882 S$ \\
\hline Alexa-546 Donkey anti-mouse IgG & Thermo Fisher Scientific & A10036 \\
\hline Alexa-546 Donkey anti-rabbit IgG & Thermo Fisher Scientific & A10040 \\
\hline HRP Goat anti-mouse IgG & Jackson Immunoresearch & $115-035-146$ \\
\hline HRP Goat anti-rabbit IgG & Jackson Immunoresearch & $111-035-144$ \\
\hline
\end{tabular}




\section{Human ESC culture}

The female hESC line WA\#22 was obtained from the WiCell Research Institute (Madison, WI, USA) and maintained on mitomycin inactivated mouse embryonic fibroblast (MEF) layers. HESC media contained DMEM/F12, 15\% human ESC-qualified fetal bovine serum (Biological Industries), 5\% KnockOut Serum replacement, 1x non-essential aminoacids, 1x Glutamax, $50 \mu \mathrm{M}$ Beta-mercaptoethanol (all from Thermo Fisher Scientific) supplemented with $5 \mathrm{ng} / \mathrm{mL}$ basic FGF (Miltenyi). $1 \mathrm{mg} / \mathrm{mL}$ Collagenase (Thermo Fisher Scientific) was used to passage hESCs.

\section{Mouse experiments}

MEFs and brain samples were isolated from embryonic day 13.5 old mouse embryos. Animal experiments were performed in accordance with protocols approved by the Animal Care Committee of the University of Oslo.

\section{Generation of genetically modified WA\#22 hESCs}

Targeted knock-out of the OPA1 gene was achieved using CRISPR-Cas9 technology. Human codon optimized SpCas9-expressing px330-U6-Chimeric_BB-CBh-hSpCas9 plasmid was a gift from Feng Zhang (Addgene plasmid \# 42230). Oligonucleotides were designed using the http://crispr.mit.edu/ webtool, and annealed oligos were cloned into px330 plasmid according to the published protocol (Cong et al., 2013). Guide RNAs (sg1: 5'GCGACTACGTCGGGCCGCTG-3'; sg2: 5'-GCTGGGCTTACGTTCGGAGC-3') were used to target the human OPA1 locus. hESCs were cultured in Rho Kinase (ROCK)-inhibitor (Y-27632; ATCC) 24 hours prior to electroporation. Cells were harvested using $0.25 \%$ trypsin/ EDTA solution (Thermo Fisher Scientific) and $1 \times 107$ cells resuspended in $500 \mu$ l Opti-MEM (Thermo Fisher Scientific) were electroporated with $15 \mu \mathrm{g}$ of each CRISPR plasmid (200 V, $500 \mu \mathrm{F}, 0.4$ $\mathrm{cm}$ cuvettes). Cells were subsequently plated on MEF feeder layers in hESC medium supplemented with ROCK-inhibitor for 48 hours. One week after electroporation, individual colonies were picked and expanded. OPA1 deficient hESC clones were verified by PCR analysis and sequencing as described below. Two independent lines that slightly differed in their cut and repair mutation (OPA1+/- hESC \#42 and \#63) were derived. The majority of experiments were performed using clone \#42. Key results were confirmed using clone \#63 as indicated.

\section{Genotyping}

WA\#22 cells were washed in PBS and lysed in $500 \mu$ INA lysis buffer (100 mM Tris pH 8.0, 5 $\mathrm{mM}$ EDTA, $0.2 \%$ SDS, $200 \mathrm{mM} \mathrm{NaCl}, 200 \mu \mathrm{g} / \mathrm{ml}$ proteinase $\mathrm{K}$ ) overnight at $55^{\circ} \mathrm{C}$. DNA was precipitated using 100\% isopropanol and washed with $70 \%$ ethanol. Genomic DNA was resuspended in $10 \mathrm{mM}$ Tris $\mathrm{pH} 8.0,0.1 \mathrm{mM}$ EDTA and incubated at $55^{\circ} \mathrm{C}$ overnight before subjected to PCR analysis. To verify deletion of OPA1 exon 1, forward (FW) primer (5'-GGC ATC TCG AAT CAC CGT CTC TGA CAC C-3') and reverse (RV) primer (5'-GGT GCC TGT CAG GCT CTT GCG G-3') were used (wt: 750 bp; targeted allele: 450 bp).

\section{Reprogramming of patient blood samples}

Mononuclear cells were isolated from $20 \mathrm{~mL}$ of peripheral blood using Ficoll-Hypaque density gradient centrifugation as described (Ferrante and Thong, 1980). Blood was isolated from two male patients heterozygous for the OPA1 c.2873_2876delTTAG deletion (transcript ID: ENST00000361510.7), which causes a V958G/R959D substitution followed by a premature stop codon. 1x 106 MNC were cultured in alpha-MEM-10\% FBS containing $20 \mathrm{ng} / \mathrm{mL}$ of each, thrombopoietin, stem cell factor, Flt3 ligand and interleukin- 6 for 2 days before being transduced using OKSM Sendai virus (CytoTune ${ }^{\mathrm{TM}}$-iPS 2.0 (Thermo Fisher Scientific)) and spin infection (1650 rpm, $45 \mathrm{~min}$ ). After centrifugation, cells were added to mitomycin-C inactivated MEFs, and hESC media was changed every 2 days. Up to five individual iPSC colonies were picked and 
expanded from each of the two donors. The presence of the OPA1 c.2873_2876delTTAG deletion was confirmed by sequencing. Samples were obtained following informed consent using protocols conformed to the principles set out in the WMA Declaration of Helsinki, and approved by the Regional Medical and Health Research Ethics Committee of South-East Norway.

\section{Copy number analysis}

High resolution sequencing was performed to detect genome wide copy number alterations (CNAs) in DNA samples isolated from hESCs. Quality control of the raw sequence data was performed using FastQC v0.11.3. The reads were aligned with Bowtie v2.2.6 against the human reference genome hg19 using the --sensitive preset option (Nasca et al., 2017). Concordantly aligned read pairs and reads with the mapping quality of at least 30 were extracted using SAMtools v1.5 ( $\mathrm{Li}$ et al., 2009). Copy number alterations (CNAs) were detected using QDNAseq v.1.10.0 with a window size of $100 \mathrm{kbp}$ (Scheinin et al., 2014), excluding the sex chromosomes $X$ and Y. No high confidence CNAs were detected in OPA1+/+ and OPA1+/- hESCs (data not shown).

\section{Neural differentiation}

Neural differentiation was performed using dual-SMAD inhibition as described previously (Chambers et al., 2009, Shi et al., 2012). Briefly, single cell suspensions of hESCs were prepared using Accutase (Thermo Fisher Scientific) and 1x106 cells were seeded into Matrigel (Corning) coated 12-well tissue-culture plates (Falcon) in MEF-conditioned hESC media supplemented with $20 \mathrm{ng} / \mathrm{mL}$ basic FGF and $10 \mu \mathrm{M}$ Rock inhibitor. Next day media was changed to neural induction media supplemented with $10 \mu \mathrm{M}$ SB431542 and $1 \mu \mathrm{M}$ Dorsomorphin (both from Sigma). Rock inhibitor was withdrawn at day 2. At day 11, neuroepithelial layers were either harvested for analysis or dissociated into small clusters with cell scraper/P1000 tips, and passaged into poly-LLysine (PLL)/Laminin (both from Sigma) coated plates. NPCs were cultured in neural induction media supplemented with SB431542/Dorsomorphin until observance of neural rosettes with everyday media change. At day 15-16, when rosette structures started to appear, $20 \mathrm{ng} / \mathrm{mL}$ basic FGF was also added to neural induction media for 3 days to promote NPC survival and proliferation. At day 21, NPCs were harvested for downstream analysis or used for neuronal differentiation. For the latter, day 21 NPCs were harvested using Accutase (Life technologies) and seeded at 20,000 cells / $\mathrm{cm}_{2}$ density on PLL / Laminin coated 6 well plates (Corning). Following a total of 3-6 weeks, N2/B27 neural differentiation media was changed every 2-4 days and successful terminal neuronal differentiation was determined by morphological changes under bright-field microscope or immunostainings with neuronal markers.

Rescue experiments were performed using the DNA methyltransferase inhibitor RG108 (Sigma). Before neural differentiation, hESCs were incubated for 7 days with different concentration of RG108 in hESC media, and the RG108 was also added during neural differentiation. Media was changed every day.

\section{Protein extraction and immunoblotting}

Total cell lysates were prepared with cell lysis buffer $(10 \mathrm{mM}$ Tris $\mathrm{pH} 7.5,150 \mathrm{mM} \mathrm{NaCl}, 1 \mathrm{mM}$ EDTA, $1 \%$ SDS, $0.5 \%$ Na-deoxycholate) supplemented with protease inhibitor (Thermo Fisher Scientific) and samples were sonicated at $4^{\circ} \mathrm{C}$ to shear DNA. Protein concentration was measured using BCA (Thermo Fisher Scientific) and equal amounts of proteins were loaded on 4-20\% gradient gels (Bio-Rad) in SDS running buffer (25 mM Tris-base, $192 \mathrm{mM}$ glycine, 0.1\% SDS). Proteins were transferred into Immobilon-P PVDF membranes in transfer buffer $(25 \mathrm{mM}$ Trisbase, $192 \mathrm{mM}$ glycine, 10\% methanol). Membranes were blocked with 5\% milk / 0.1\% Tween-20 in TBS buffer (25 mM Tris-base, $137 \mathrm{mM} \mathrm{NaCl}, 2.7 \mathrm{mM} \mathrm{KCl}, \mathrm{pH}$ was adjusted to 7.4) and incubated overnight with primary antibodies $(1 \mu \mathrm{g} / \mathrm{mL})$ at $4^{\circ} \mathrm{C}$. Respective HRP-linked secondary antibodies (Jackson Immunoresearch) were used at $1 \mu \mathrm{g} / \mathrm{mL}$ concentration in blocking buffer for 
4 hours at room temperature. Chemiluminescent imaging was performed using Bio-Rad Imaging system. Quantification of western blots was done using Bio-Rad software. Primary and secondary antibodies are listed in Table S5.

\section{Analysis of mitochondrial morphology and DNA content}

To analyze cellular mitochondrial distribution, hESCs were dissociated as single cells using Accutase (Thermo Fisher Scientific) and seeded on Matrigel coated plates in MEF-conditioned hESC media supplemented with $20 \mathrm{ng} / \mathrm{mL}$ basic FGF and $10 \mu \mathrm{M}$ ROCK-inhibitor. Next day, cells were incubated overnight with $2 \mu \mathrm{L}$ of Celllight mitochondria-GFP, BacMam 2.0 (mito-GFP) reagent per 10.000 cells according to the manufacturer's instructions. After 18 hours, cells were fixed with $2 \%$ methanol-free formaldehyde (Thermo Fisher Scientific) for 15 min. After washing twice with PBS, coverslips were mounted with ProLong Diamond antifade mountant (Thermo Fisher Scientific). Photographs were taken using a Zeiss LSM 800 confocal laser scanning microscope. Images were processed using Zeiss Zen software Blue edition.

To measure mitochondrial DNA content of cells, DNA was extracted using DNeasy Blood \& Tissue kit (Qiagen) according to the manufacturer's instructions. DNA concentration was measured using Nanodrop instrument. Real-time PCR was performed as duplicates using $10 \mathrm{ng}$ DNA for each reaction. Mitochondrial genes NADH-ubiquinone oxidoreductase chain 1 (MT-ND1) and MT-ND4 genes were quantified and normalized to nuclear gene beta-2-microglobulin (B2M) to measure mitochondrial:nuclear DNA copy number ratio.

\section{Mitochondrial Respiration and ROS Measurements}

Oxygen consumption rate (OCR) was determined using the Seahorse XF Cell Mito Stress Test and Seahorse XFe96 Analyzer (Agilent). In brief, hESCs were seeded onto a Matrigel-coated XF96 cell culture microplate (Agilent) at a density of 20,000 cells per well and incubated at $37{ }^{\circ} \mathrm{C}$ overnight in hESC medium supplemented with $10 \mu \mathrm{M}$ ROCK inhibitor. One hour before the analysis, culture medium was changed to Seahorse XF DMEM Medium, pH 7.4 (Agilent) supplemented with $2 \mathrm{mM}$ glutamine, $1 \mathrm{mM}$ pyruvate and $10 \mathrm{mM}$ glucose.

Week 3 NPCs were harvested using Accutase and seeded onto a Matrigel-coated XF96 cell culture microplate at a density of 20,000 cells per well. Cells were cultured overnight in N2/B27 media before OCR measurements were performed as described above. To assess mitochondrial respiration in neurons, NPCs were initially seeded at 20,000 cells / $\mathrm{cm}_{2}$ density in 6 well plates and differentiated into immature neurons for 7 - 10 days in N2/B27 media. Immature neurons were harvested using Accutase and seeded onto a Matrigel-coated XF96 cell culture microplate at a density of 10,000 cells per 96 well. Neurons were further differentiated for 2 weeks in N2 /B27 media in XF96 cell culture microplates before measuring oxygen consumption rates.

Selective inhibitors of mitochondrial electron transport chain: Oligomycin $(1 \mu \mathrm{M})$, carbonyl cyanide $\mathrm{p}$-(trifluoromethoxy) phenylhydrazone $(\mathrm{FCCP})(2 \mu \mathrm{M})$, and rotenone/antimycin $\mathrm{A}(0.5 \mu \mathrm{M})$ were injected sequentially during the measurements at indicated times to assess different parameters of mitochondrial functions. Basal respiration was determined as OCR before injection of oligomycin minus OCR after injection of rotenone/antimycin A. ATP-linked respiration (here noted as ATP production) was determined as OCR before oligomycin injection minus OCR after oligomycin treatment. Maximal respiration was determined as the OCR after FCCP minus nonmitochondrial OCR (OCR after rotenone/antimycin A treatment). Values were normalized to protein concentrations, which were determined using the BCA protein assay kit (Thermo Fisher Scientific).

ROS levels were measured using the general oxidative stress indicator 5-(and 6-) chloromethyl2',7'-dichlorodihydrofluorescein diacetate, acetyl ester (CM-H2DCFDA) (Thermo Fisher Scientific) 
according to the manufacturer's instructions. Single cell suspensions were loaded with $2 \mu \mathrm{M} C \mathrm{CM}$ H2DCFDA for 20 min in a tissue culture incubator. After washing with PBS, cells were analyzed immediately using the BD FACSCanto flow cytometer and Kaluza software (Beckman Coulter). Arithmetic mean values of fluorescence intensity were used to quantify ROS levels.

\section{Flow cytometry analysis of cell cycle and proliferation}

Cell cycle analysis and proliferation were assessed using fluorescent DNA intercalating agent propidium iodide (PI) and Alexa Flour 488 - conjugated Ki-67 antibody. Single cell suspensions were fixed overnight at $-80{ }^{\circ} \mathrm{C}$ with $90 \%$ ethanol / $\mathrm{dH} 20$. Cells were permeabilized with $0.25 \%$ Triton / PBS for 15 minutes and incubated with blocking solution 0.5\% BSA / 0.1\% Tween / PBS. Cells were incubated with Ki-67 antibody for 1 hour in dark at room temperature followed by incubation with $100 \mu \mathrm{g} / \mathrm{mL}$ RNase A for $15 \mathrm{~min}$ at $37^{\circ} \mathrm{C}$ to block non-specific PI binding. Subsequently, DNA was labeled with $100 \mu \mathrm{g} / \mathrm{mL}$ PI for $15 \mathrm{~min}$ in dark at room temperature. After washing with PBS, cells were analyzed immediately using the BD FACSCanto flow cytometer. Kaluza Analysis Software (Beckman Coulter) was used for analysis and illustration of flow cytometer data.

\section{Flow cytometry analysis of apoptosis}

Analysis of early and late apoptosis was carried out using FITC Annexin V Apoptosis Detection Kit (BD Pharmingen) according to the manufacturer's instructions. Single cell suspensions in binding buffer were loaded with $5 \mu \mathrm{l}$ Annexin $\mathrm{V}$ and $5 \mu \mathrm{l}$ PI solutions for $15 \mathrm{~min}$ in dark at room temperature. To address sensitivity to apoptotic stimuli, cells were incubated with $50 \mathrm{mM}$ hydrogen peroxide solution for 30 min (neurons) or 45 min (NPCs). Cells were washed with binding buffer before dye loading is performed. After washing with binding buffer, cells were analyzed immediately using the BD FACSCanto flow cytometer. Kaluza Analysis Software (Beckman Coulter) was used for analysis and illustration of flow cytometer data.

\section{Live cell automated imaging}

To measure the cell proliferation rate, hESCs were dissociated to single cells using Accutase (Thermo Fisher Scientific). After, an equal number of cells was seeded into Matrigel (Corning) coated 24-well tissue-culture plates (Falcon) in MEF-conditioned hESC media supplemented with $20 \mathrm{ng} / \mathrm{mL}$ basic FGF and $10 \mu \mathrm{M}$ Rock inhibitor. Next day, the plate was placed in a tissue culture incubator equipped with an IncuCyte Live Cell Analysis System. The proliferation rate was analyzed using the IncuCyte Zoom software.

\section{Immunostaining}

Cells were fixed with $2 \%$ methanol-free formaldehyde (Thermo Fisher Scientific) for 15 min and permeabilized with $0.3 \%$ Triton/PBS for $10 \mathrm{~min}$ at room temperature. Cells were blocked in $1 \%$ BSA $2 \%$ FBS/ $0.1 \%$ Tween/ PBS solution for 30 minutes at room temperature and incubated with primary antibodies $\left(5-8 \mu \mathrm{g} / \mathrm{mL}\right.$ in PBS) overnight at $4^{\circ} \mathrm{C}$ or for 3 hours at room temperature. After PBS washings, samples were incubated with the respective Alexa-488 or Alexa-546 conjugated secondary antibodies (Thermo Fisher Scientific) used at $5 \mu \mathrm{g} / \mathrm{mL}$ concentration for 3 hours at room temperature and nuclei were stained with DAPI. Samples were mounted with ProLong Diamond antifade mountant (Thermo Fisher Scientific). Photographs were taken with Zeiss Axio Vert.A1 microscope equipped with an AxioCam MRm camera or Zeiss LSM 800 confocal laser scanning microscope. Images were processed using Zeiss Zen software Blue edition. Primary and secondary antibodies are listed in Table S5.

\section{RNA isolation and $q R T-P C R$}


Cells were lysed with Trizol reagent (Qiagen) and RNA was extracted with chloroform. Extracted RNA was further cleaned using the RNAeasy kit (Qiagen) and on-column DNA digestion according to the manufacturer's instructions. The ABI cDNA synthesis kit was used to transcribe $1 \mu \mathrm{g}$ of RNA into cDNA. Real-time PCR reactions were prepared with $10 \mathrm{ng}$ of cDNA, $1 \times$ SYBR green, and $1 \mu \mathrm{M}$ of each primer. Samples were run as technical replicates in an ABI9600 Instrument or BioRAD instrument. Specificity of amplification was shown using Melt-Curve, and samples were analyzed using $2.5 \%$ agarose gels. The expression value was calculated based on the Delta-Delta-CT method and normalized to expression of the housekeeping gene 18S rRNA. Primer sequences are listed in Table S4.

\section{Statistical analysis}

Visualization and statistical analysis of data was performed using GraphPad Prism. Data is presented as mean \pm standard error of mean (SEM). Student's t-test was used to analyze the differences between groups. ${ }^{*}=p<0.05,{ }^{* *}=p<0.01,{ }^{* * *}=p<0.001$

\section{Patch clamp experiments}

One coverslip with hESC-derived neurons was taken out of the incubator and transferred to a recording chamber where the cells were submerged in artificial cerebrospinal fluid (aCSF) (4.5 $\mathrm{ml} / \mathrm{min}, 32 \pm 1^{\circ} \mathrm{C}$ ). The aCSF contained (in $\mathrm{mM}$ ): $124 \mathrm{NaCl}, 2 \mathrm{KCl}, 1.25 \mathrm{KH}_{2} \mathrm{PO}_{4}, 2 \mathrm{MgSO}_{4}, 2$ $\mathrm{CaCl}_{2}, 26 \mathrm{NaHCO}_{3}, 12$ glucose (all from Sigma-Aldrich) and bubbled with a mixture of $95 \%$ $\mathrm{O}_{2}$ and $5 \% \mathrm{CO}_{2}$. Visual guided patch clamping was performed with an Olympus microscope (BX51WI) with DIC optics and fitted with recording chamber and manipulators from Luigs and Neumann. Recording electrodes (Borosilicate 6-9 Mohm, O.D. $1.5 \mathrm{~mm}$ I.D. $0.86 \mathrm{~mm}$ with filament, Sutter Instrument) were filled with intracellular solution (120 mM potassium gluconate, $20 \mathrm{mM}$ $\mathrm{KCl}, 10 \mathrm{mM}$ EGTA, $2 \mathrm{mM} \mathrm{MgCl}$, $2 \mathrm{mM} \mathrm{Na} 2 \mathrm{ATP}, 10 \mathrm{mM}$ HEPES, pH adjusted to 7.3 with $\mathrm{KOH}$ ). Axon Multiclamp 700B amplifier, Digidata 1440 and pClamp10 software (Molecular Devices) were used for recording and digitalizing the electrophysiological signals.

\section{Transmission electron microscopy}

hESC pellets were fixed in $2.5 \%$ glutaraldehyde in $0.1 \mathrm{M}$ phosphate buffer overnight, and the pellets were subsequently post-fixed with $2 \%$ osmium tetroxide in $0.2 \mathrm{M}$ cacodylate buffer. The cells were dehydrated with increasing concentration of ethanol, stained en bloc with $2 \%$ uranyl acetate in absolute ethanol. The final dehydration was carried out by two shifts of acetone. Then the pellets were infiltrated with mixtures of acetone and epoxy resin (Agar 100, Agar Scientific Itd): $33 \%$ epoxy, 50\% epoxy, 67\% epoxy and finally pure epoxy resin. The cell pellets were embedded in their Eppendorf tubes and polymerized overnight at $60^{\circ} \mathrm{C}$.

Ultrathin sections of $70 \mathrm{~nm}$ were cut with an ultramicrotome (EMUC6, Leica), and mounted onto formvar-coated $0.75 \times 2 \mathrm{~mm}$ slot grids, and subsequently stained with $4 \%$ uranyl acetate in $40 \%$ ethanol and Reynolds lead citrate. The sections were examined in an electron microscope (Tecnai Spirit Biotwin). For each cell population mitochondria from 10 cells were digitally photographed at a magnification of 11000 . The average length of the mitochondria was measured using analySIS $\circledast$ (Olympus) software.

Samples for measurements of mitochondrial circumference and intercristae distance were fixed in $2 \%$ glutaraldehyde in $0.1 \mathrm{M}$ PHEM buffer, followed by post-fixation in $2 \% \mathrm{OsO}_{4}$ and $1 \%$ potassium ferricyanide, and en bloc staining with $0.5 \%$ tannic acid and $1 \%$ Uranyl acetat. Dehydration was performed in ascending ethanol series followed by embedding in Epon. Ultrathin sections (80-100 nm) were cut on a Leica Ultracut and collected onto formvar-coated 100 mesh hexagonal grids. Images were acquired in a JEOL-JEM 1230 at $80 \mathrm{kV}$ and recorded with a Morada camera (Olympus, Germany). For measuring mean circumference of mitochondria, images were taken at $15 \mathrm{kx}$ in a systematic, uniform random fashion. Line intersections with the mitochondrial membrane were counted using a test lattice of $300 \mathrm{~nm}$ sidelength. 65 and 57 mitochondria were 
counted from $O P A 1+/+$ and $O P A 1+/-$ hESCs, respectively. $\mathrm{N}=2$ experiments. Interchristae distances were determined counting $\mathrm{N}=52$ distances in $O P A 1+/+$ and $\mathrm{N}=105$ distances in $O P A 1+/$ - conditions.

\section{RNA sequencing}

Integrity of the RNA samples was determined with Bioanalyzer (Agilent) and samples with an RNA Integrity number $>9$ were used for sequencing. Libraries were prepared using Strandspecific TruSeq RNA-seq Sample Preparation Kit v2 (Illumina). Paired-end sequencing with 150 bp read-length was performed with a HiSeq 3000 machine.

Raw reads were aligned to the human genome (hg19), and splice junctions were mapped using TopHat v2.1.0 (Trapnell et al., 2012) with default parameters. Gene based read counts were obtained using featureCount v1.5.0-p1 (Liao et al., 2014) using default parameters. To perform normalization and statistical evaluation with negative binomial model between conditions on the count data, we used DESeq2 v1.10.1 Biconductor package (Love et al., 2014). Genes were considered differentially expressed when showing an absolute $\log _{2}$ fold change (treatment/control) $\geq 1.5$ with adjusted $p$-value $($ padj $) \leq 0.05$.

\section{Bisulfite sequencing}

DNA was prepared using DNeasy Blood \& Tissue kit (Qiagen) and bisulfite conversion was performed with MethylEdge Bisulfite Conversion System (Promega) according to the manufacturer's protocol. Primers for PCR amplification of CpG island in FOXG1 5'UTR and ATP10D promoter were designed using MethPrimer program (Li and Dahiya, 2002). Primer sequences are listed in Table S4. PCR amplification of the target region in bisulfite converted DNA was achieved using EpiMark Hot Start Taq DNA polymerase (New England Biolabs) with 15-20 ng template DNA and confirmed by agarose gel electrophoresis.

Fresh PCR product $(4 \mu \mathrm{L})$ was cloned into pCR4-TOPO (Thermo Fisher Scientific) vector according to the manufacturer's protocol. Briefly, $4 \mu \mathrm{L}$ PCR product $+1 \mu \mathrm{L}$ salt solution $+1 \mu \mathrm{L}$ TOPO vector were mixed and incubated for $15 \mathrm{~min}$ at room temperature. Blue-white selection of transformed DH5alpha competent cells was done on IPTG/X-Gal $(1 \mathrm{mM} / 20 \mu \mathrm{g} / \mathrm{mL})$ coated ampicillin selection $(30 \mu \mathrm{g} / \mathrm{mL}$ ) agar plates. White colonies were expanded in liquid LB media with $30 \mu \mathrm{g} / \mathrm{mL}$ ampicillin. Plasmids were prepared using Genejet Plamid Miniprep kit and sequenced using M13rev (-29) primer.

\section{Quantification of DNA modifications and cellular metabolites using LC-MS/MS}

DNA was added internal standard d3-5-hydroxymethyl-2'-deoxycytidine (Toronto Research Chemicals) and hydrolyzed to deoxynucleosides by $20 \mathrm{U}$ benzonase (Santa Cruz Biotech), $0.2 \mathrm{U}$ nuclease P1, and $0.1 \mathrm{U}$ alkaline phosphatase (Sigma) in $10 \mathrm{mM}$ ammonium acetate $\mathrm{pH} 6.0$ and $1 \mathrm{mM}$ magnesium chloride at $40{ }^{\circ} \mathrm{C}$ for $45 \mathrm{~min}$, then added 3 volumes of acetonitrile and centrifuged $\left(16,000 \mathrm{~g}, 30 \mathrm{~min}, 4^{\circ} \mathrm{C}\right)$. The supernatants were dried and dissolved in $50 \mu \mathrm{l}$ water for LC-MS/MS analysis of modified and unmodified deoxyribonucleosides. Chromatographic separation was performed using an Agilent 1290 Infinity II UHPLC system with a ZORBAX RRHD Eclipse Plus C18 $150 \times 2.1 \mathrm{~mm}$ ID $(1.8 \mu \mathrm{m})$ column protected with a ZORBAX RRHD Eclipse Plus C18 $5 \times 2.1 \mathrm{~mm}$ ID $(1.8 \mu \mathrm{m})$ guard column (Agilent). The mobile phase consisted of water and methanol (both added $0.1 \%$ formic acid), for $5-\mathrm{hm}(\mathrm{dC})$ initially run at $0.15 \mathrm{~mL} / \mathrm{min}$ with $5 \%$ methanol for $0.5 \mathrm{~min}$, followed by a 4-min gradient of 5-15\% methanol, a flow increase to 0.25 $\mathrm{mL} / \mathrm{min}$ and a 3 min gradient of $15-90 \%$ methanol, then 4 min re-equilibration with $5 \%$ methanol. A portion of each sample was diluted for the analysis of 5-methyl(dC) and unmodified deoxynucleosides. 5-methyl( $\mathrm{dC}$ ) was chromatographed at $0.25 \mathrm{ml} / \mathrm{min}$ with a 3 -min gradient of 5$90 \%$ methanol, followed by 4 min re-equilibration with $5 \%$ methanol, and unmodified deoxynucleosides were chromatographed isocratically with $20 \%$ methanol. Mass spectrometric detection was performed using an Agilent 6495 Triple Quadrupole system operating in positive 
electrospray ionization mode, monitoring the mass transitions $261.1 / 145.1$ (d $3-5-h m(d C)$ ), 258.1/142.1 (5-hm(dC)), 242.1/126.1 (5-m(dC)), 252.1/136.1 (dA), 228.1/112.1 (dC), 268.1/152.1 $(\mathrm{dG})$, and 243.1/127.1 (dT).

S-adenosylmethionine (SAM) and S-adenosylhomocysteine (SAH) were measured by LC-MS/MS according to the method of Klepacki et al (Klepacki et al., 2013) with modifications optimized for cell lysates. Briefly, $100 \mu \mathrm{L}$ cell lysate made up in $0.1 \%$ formic acid (FA) was mixed with $100 \mu \mathrm{L}$ internal standard mix $\left(5 \mu \mathrm{M}_{2} \mathrm{H}_{3}-\mathrm{SAM}\right.$ and $\left.5 \mu \mathrm{M}{ }_{13} \mathrm{C}_{5}-\mathrm{SAH}\right)$, also prepared in $0.1 \% \mathrm{FA}$. Samples were vortexed for 5 minutes followed by incubation on ice for 10 minutes. Extracted samples were then centrifuged at $13,000 \mathrm{rpm}$ for $10 \mathrm{~min}$ and $60 \mu \mathrm{L}$ supernatant was transferred to HPLC vials and $4 \mu \mathrm{L}$ were injected into the LC-MS/MS system. SAM and SAH were separated on a Nexera N2 (Shimadzu) equipped with a SunFire C8, 4.6 × $100 \mathrm{~mm}$ and $3.5 \mu \mathrm{m}$ particle size column (Waters $\mathrm{GmbH}$, Eschborn) under isocratic conditions and mass transitions were monitored with a QTrap $6500+$ (Sciex, Darmstadt). A flow of $0.75 \mathrm{~mL} /$ minute of $5 \%$ Methanol and $95 \%$ Water (both containing $0.1 \% \mathrm{FA}$ ) was used to analyze SAM and SAH. The protonated molecular ions $[\mathrm{M}+\mathrm{H}]+$ were: SAM $(\mathrm{m} / \mathrm{z}=399.0),{ }_{2} \mathrm{H}_{3}-\mathrm{SAM}(\mathrm{m} / \mathrm{z}=402.0), \mathrm{SAH}(\mathrm{m} / \mathrm{z}=385.1)$ and ${ }_{13} \mathrm{C}_{5}-\mathrm{SAH}(\mathrm{m} / \mathrm{z}$ $=390.0)$. The product ions were: $\mathrm{SAM}(\mathrm{m} / \mathrm{z}=250.1$ and $\mathrm{m} / \mathrm{z}=136.2), 2 \mathrm{H}_{3}-\mathrm{SAM}(\mathrm{m} / \mathrm{z}=250.1$ and $\mathrm{m} / \mathrm{z}=136.2)$, $\mathrm{SAH}(\mathrm{m} / \mathrm{z}=136.2)$, and ${ }_{13} \mathrm{C}_{5} \mathrm{SAH}(\mathrm{m} / \mathrm{z}=137.2)$. Quantification was carried by a calibration curve prepared under the exact same conditions. The linear range of calibrators under our experimental conditions is $0-1200 \mathrm{nM}$ for both metabolites. SAM and SAH concentrations were normalized by the total concentration of protein in the lysate. Total protein concentration was determined with the BCA assay (Thermo Fisher Scientific).

Alpha-ketoglutarate and succinate were quantified according to a published LC-MS/MS method (Mullen et al., 2014) with modifications in sample preparation as follows: Cells were harvested as described above and washed with PBS prior to storage at $-80 \circ \mathrm{C}$ as dry cell pellets. Dry cell pellets were resuspended with aqueous $0.1 \% \mathrm{FA}$ and subjected to three cycles of freeze-thawing by alternating dry-ice and water-bath incubation at $25 \circ \mathrm{C}$. Subsequent vortexing, concentration, metabolite reconstitution, sample running and analysis were performed as described in the literature (Mullen et al., 2014). A twelve-point calibration curve was utilized to quantify each metabolite with a linear range of 0 to $500 \mu \mathrm{M}$.

\section{References}

AKEPATI, V. R., MULLER, E. C., OTTO, A., STRAUSS, H. M., PORTWICH, M. \& ALEXANDER, C. 2008. Characterization of OPA1 isoforms isolated from mouse tissues. J Neurochem, 106, 372-83.

CHAMBERS, S. M., FASANO, C. A., PAPAPETROU, E. P., TOMISHIMA, M., SADELAIN, M. \& STUDER, L. 2009. Highly efficient neural conversion of human ES and iPS cells by dual inhibition of SMAD signaling. Nat Biotechnol, 27, 275-80.

CONG, L., RAN, F. A., COX, D., LIN, S., BARRETTO, R., HABIB, N., HSU, P. D., WU, X., JIANG, W., MARRAFFINI, L. A. \& ZHANG, F. 2013. Multiplex Genome Engineering Using CRISPR/Cas Systems. Science, 339, 819-823.

DELETTRE, C., GRIFFOIN, J. M., KAPLAN, J., DOLLFUS, H., LORENZ, B., FAIVRE, L., LENAERS, G., BELENGUER, P. \& HAMEL, C. P. 2001. Mutation spectrum and splicing variants in the OPA1 gene. Hum Genet, 109, 584-91.

DELETTRE, C., LENAERS, G., GRIFFOIN, J. M., GIGAREL, N., LORENZO, C., BELENGUER, P., PELLOQUIN, L., GROSGEORGE, J., TURC-CAREL, C., PERRET, E., ASTARIE-DEQUEKER, C., LASQUELLEC, L., ARNAUD, B., DUCOMMUN, B., KAPLAN, J. \& HAMEL, C. P. 2000. Nuclear gene OPA1, encoding a mitochondrial dynamin-related protein, is mutated in dominant optic atrophy. Nat Genet, 26, 207-10. 
FERRANTE, A. \& THONG, Y. H. 1980. Optimal conditions for simultaneous purification of mononuclear and polymorphonuclear leucocytes from human blood by the hypaque-ficoll method. Journal of Immunological Methods, 36, 109-117.

KLEPACKI, J., BRUNNER, N., SCHMITZ, V., KLAWITTER, J., CHRISTIANS, U. \& KLAWITTER, J. 2013. Development and validation of an LC-MS/MS assay for the quantification of the trans-methylation pathway intermediates S-adenosylmethionine and S-adenosylhomocysteine in human plasma. Clinica Chimica Acta, 421, 91-97.

LI, H., HANDSAKER, B., WYSOKER, A., FENNELL, T., RUAN, J., HOMER, N., MARTH, G., ABECASIS, G., DURBIN, R. \& GENOME PROJECT DATA PROCESSING, S. 2009. The Sequence Alignment/Map format and SAMtools. Bioinformatics, 25, 2078-2079.

LI, L.-C. \& DAHIYA, R. 2002. Methprimer: Designing Primers for Methylation PCRs.

LIAO, Y., SMYTH, G. K. \& SHI, W. 2014. featureCounts: an efficient general purpose program for assigning sequence reads to genomic features. Bioinformatics, 30, 923-30.

LOVE, M. I., HUBER, W. \& ANDERS, S. 2014. Moderated estimation of fold change and dispersion for RNA-seq data with DESeq2. Genome Biol, 15, 550.

MULLEN, ANDREW R., HU, Z., SHI, X., JIANG, L., BOROUGHS, LINDSEY K., KOVACS, Z., BORIACK, R., RAKHEJA, D., SULLIVAN, LUCAS B., LINEHAN, W. M., CHANDEL, NAVDEEP S. \& DEBERARDINIS, RALPH J. 2014. Oxidation of Alpha-Ketoglutarate Is Required for Reductive Carboxylation in Cancer Cells with Mitochondrial Defects. Cell Reports, 7, 1679-1690.

NASCA, A., RIZZA, T., DOIMO, M., LEGATI, A., CIOLFI, A., DIODATO, D., CALDERAN, C., CARRARA, G., LAMANTEA, E., AIELLO, C., DI NOTTIA, M., NICETA, M., LAMPERTI, C., ARDISSONE, A., BIANCHI-MARZOLI, S., IAROSSI, G., BERTINI, E., MORONI, I., TARTAGLIA, M., SALVIATI, L., CARROZZO, R. \& GHEZZI, D. 2017. Not only dominant, not only optic atrophy: expanding the clinical spectrum associated with OPA1 mutations. Orphanet J Rare Dis, 12, 89.

SCHEININ, I., SIE, D., BENGTSSON, H., VAN DE WIEL, M. A., OLSHEN, A. B., VAN THUIJL, H. F., VAN ESSEN, H. F., EIJK, P. P., RUSTENBURG, F., MEIJER, G. A., REIJNEVELD, J. C., WESSELING, P., PINKEL, D., ALBERTSON, D. G. \& YLSTRA, B. 2014. DNA copy number analysis of fresh and formalin-fixed specimens by shallow whole-genome sequencing with identification and exclusion of problematic regions in the genome assembly. Genome Research.

SHI, Y., KIRWAN, P. \& LIVESEY, F. J. 2012. Directed differentiation of human pluripotent stem cells to cerebral cortex neurons and neural networks. Nat Protoc, 7, 1836-46.

trapnell, C., ROBERTS, A., GOFF, L., PERTEA, G., KIM, D., KELlEY, D. R., PIMENTEL, H., SALZBERG, S. L., RINN, J. L. \& PACHTER, L. 2012. Differential gene and transcript expression analysis of RNA-seq experiments with TopHat and Cufflinks. Nat Protoc, 7, 562-78. 